\title{
AVERAGES OF CHARACTERISTIC POLYNOMIALS IN RANDOM MATRIX THEORY
}

\author{
A. BORODIN AND E. STRAHOV
}

\begin{abstract}
We compute averages of products and ratios of characteristic polynomials associated with Orthogonal, Unitary, and Symplectic Ensembles of Random Matrix Theory. The pfaffian/determinantal formulas for these averages are obtained, and the bulk scaling asymptotic limits are found for ensembles with Gaussian weights. Classical results for the correlation functions of the random matrix ensembles and their bulk scaling limits are deduced from these formulas by a simple computation.

We employ a discrete approximation method: the problem is solved for discrete analogues of random matrix ensembles originating from representation theory, and then a limit transition is performed. Exact pfaffian/determinantal formulas for the discrete averages are proved using standard tools of linear algebra; no application of orthogonal or skew-orthogonal polynomials is needed.
\end{abstract}

\section{INTRODUCTION}

1.1. The problem. Consider the linear space of real symmetric, Hermitian, or quaternion real Hermitian square matrices $H$ with the Gaussian measure

$$
P(d H)=\text { const } \cdot \exp \left(-\operatorname{Tr} H^{2}\right) d H
$$

These probability spaces are the basic objects of interest of Random Matrix Theory (RMT, for short); they are known as Gaussian Orthogonal, Unitary and Symplectic ensembles, respectively (GOE, GUE, and GSE). The goal of this paper is to study the averages of products and ratios of characteristic polynomials

$$
\left\langle\frac{\operatorname{det}\left(\alpha_{1}-H\right) \cdots \operatorname{det}\left(\alpha_{k}-H\right)}{\operatorname{det}\left(\beta_{1}-H\right) \cdots \operatorname{det}\left(\beta_{m}-H\right)}\right\rangle
$$

with respect to these ensembles and their generalizations.

Despite the fact that the Gaussian ensembles have been extensively studied, the progress on evaluating averages (1.1.2) remained rather limited until very recently. Starting from 1995 there appeared a number of papers by different authors where the case of unitary ensembles (exact definitions are below) was essentially settled both for the random matrices of finite size and for their scaling limits in the bulk of spectrum as the size of matrices tends to infinity, see Refs. [4, 15, 16, 45, 34, 35, 36, 30, 53, 32, 5, 2, 3, 57. The results turned out to be closely related to some problems of the classical number theory, see Refs. 20], 38, 42, for details.

Some progress have also been achieved in the orthogonal and symplectic cases: Brezin-Hikami [17. 18] computed the bulk scaling limit asymptotics of the moments $\left\langle\operatorname{det}^{k}(\alpha-H)\right\rangle$ and also provided some asymptotic expressions for averages (1.1.2) with small number of factors, and the asymptotics of the negative moments $\left\langle 1 / \operatorname{det}^{m}(\beta-H)\right\rangle$ has been obtained by Fyodorov-Keating 33] and Forrester-Keating [28].

However, the problem of computing the bulk scaling limit asymptotics of general averages (1.1.2), despite considerable interest of physicists, see e.g. Andreev-Simon 4], Gronqvist, Guhr and Kohler [37, Fyodorov [31, Szabo [54], Splttorff-Verbaarschot [52], Zirnbauer [59, 60], remained open.

The main goal of this paper is to provide explicit (determinantal or pfaffian) expressions of averages (1.1.2) in terms of those that involve only one or two determinants, and to evaluate the asymptotics of (1.1.2) in the bulk scaling limit regime of the Gaussian ensembles in the middle of the spectrum. (In the case of real symmetric matrices we consider only the case when the matrices are 
of even size.) It is worth noting that the standard correlation functions of the matrix ensembles can be easily extracted from averages (1.1.2). Thus, we obtain the classical determinantal and pfaffian formulas for the correlation functions (see e.g. Refs. [44, 27]) as a corollary.

1.2. The results: algebraic part. In order to state the results we need to introduce some notation. Take a positive measure $\mu$ on $\mathbb{R}$ with finite moments and infinite support. Then

$$
C_{N}^{(\beta)}:=\frac{1}{N !} \int_{\mathbb{R}^{N}} \prod_{1 \leq i<j \leq N}\left|x_{i}-x_{j}\right|^{\beta} \mu\left(d x_{1}\right) \otimes \cdots \otimes \mu\left(d x_{N}\right) \neq 0
$$

for any $N \geq 1$ and $\beta>0$. Take the probability measure on $\mathbb{R}^{N}$ given by

$$
p_{N}^{(\beta)}\left(d x_{1}, \ldots, d x_{N}\right)=\frac{1}{N ! C_{N}^{(\beta)}} \prod_{1 \leq i<j \leq N}\left|x_{i}-x_{j}\right|^{\beta} \mu\left(d x_{1}\right) \otimes \cdots \otimes \mu\left(d x_{N}\right) .
$$

and for any symmetric function $g: \mathbb{R}^{N} \rightarrow \mathbb{C}$ set

$$
\langle g\rangle_{\triangle_{N}^{(\beta)}}:=\int_{\mathbb{R}^{N}} g\left(x_{1}, \ldots, x_{N}\right) p_{N}^{(\beta)}\left(d x_{1}, \ldots, d x_{N}\right)
$$

provided that the integral converges. Also, for $\zeta \in \mathbb{C}$ set $D(\zeta):=\prod_{i>1}\left(\zeta-x_{i}\right)$. The number of factors in such products will always be clear from the context.

It is well known (see e.g. 44]) that the radial parts (that is, projections onto different eigenvalues $\left.\left\{x_{i}\right\}\right)$ of the probability measures

$$
P(d H)=\text { const } \cdot \exp (-Q(H)) d H,
$$

where $Q(x)$ is an even degree polynomial with positive highest coefficient, and $H$ belongs to the linear space of $2 N \times 2 N$ real symmetric, $N \times N$ Hermitian, or $N \times N$ quaternion real Hermitian matrices $^{1}$, are exactly the measures $p_{2 N}^{(1)}, p_{N}^{(2)}$, and $p_{N}^{(4)}$ with our measure $\mu$ being equal

$$
\mu(d x)= \begin{cases}\exp (-Q(x)) d x, & \beta=1,2 \\ \exp (-2 Q(x)) d x, & \beta=4\end{cases}
$$

In the cases $\beta=1$ and 2 , the characteristic polynomial $\operatorname{det}(\zeta-H)$ of the random matrix $H$ is exactly our product-function $D(\zeta)$, while in the case $\beta=4$ we have $\operatorname{det}(\zeta-H)=D^{2}(\zeta)$. For any finite sets $A=\left\{a_{1}, \ldots, a_{p}\right\}, B=\left\{b_{1}, \ldots, b_{q}\right\}$ denote

$$
\prod(A ; B)=\prod_{i=1, j=1}^{p, q}\left(a_{i}-b_{j}\right), \quad V(A)=\prod_{1 \leq i<j \leq p}\left(a_{i}-a_{j}\right), \quad V(B)=\prod_{1 \leq i<j \leq q}\left(b_{i}-b_{j}\right) .
$$

Theorem 1.2.1. (orthogonal and symplectic cases) (i) For any integers $N \geq 1$ and $S>1-N$, and finite sets of mutually distinct complex numbers

$$
\alpha=\left\{\alpha_{1}, \ldots, \alpha_{k}\right\}, \quad \beta=\left\{\beta_{1}, \ldots, \beta_{m}\right\}, \quad k-m=2 S,
$$

such that $\beta \cap \mathbb{R}=\varnothing$, one has

$$
\left\langle\frac{\prod_{i=1}^{k} D\left(\alpha_{i}\right)}{\prod_{i=1}^{m} D\left(\beta_{i}\right)}\right\rangle_{\triangle_{2 N}^{(1)}}=\frac{C_{2 N+2 S}^{(1)}}{C_{2 N}^{(1)}} \frac{\prod(\alpha ; \beta)}{V(\alpha) V(\beta)} \operatorname{Pf}\left[W_{N}^{(1)}(\alpha, \beta \mid \alpha, \beta)\right]
$$

\footnotetext{
${ }^{1}$ Matrix elements of a quaternion real matrix are $2 \times 2$ matrices of the form $\left[\begin{array}{cc}z & w \\ -\bar{w} & \bar{z}\end{array}\right]$ where $z$ and $w$ are complex numbers. An $N \times N$ quaternion real Hermitian matrix has $2 N$ eigenvalues which come in pairs of coinciding real numbers.
} 
where $W_{N}^{(1)}$ is a skew-symmetric $(k+m) \times(k+m)$ matrix with rows and columns parameterized by elements of $\alpha$ and $\beta$, and with matrix elements given by

$$
\begin{aligned}
& W_{N}^{(1)}\left(\alpha_{i}, \alpha_{j}\right)=\frac{C_{2 N+2 S-2}^{(1)}}{C_{2 N+2 S}^{(1)}}\left(\alpha_{i}-\alpha_{j}\right)\left\langle D\left(\alpha_{i}\right) D\left(\alpha_{j}\right)\right\rangle_{\triangle_{2 N+2 S-2}^{(1)}} \\
& W_{N}^{(1)}\left(\alpha_{i}, \beta_{j}\right)=\frac{1}{\alpha_{i}-\beta_{j}}\left\langle\frac{D\left(\alpha_{i}\right)}{D\left(\beta_{j}\right)}\right\rangle_{\triangle_{2 N+2 S}^{(1)}} \\
& W_{N}^{(1)}\left(\beta_{i}, \beta_{j}\right)=\frac{C_{2 N+2 S+2}^{(1)}}{C_{2 N+2 S}^{(1)}}\left(\beta_{i}-\beta_{j}\right)\left\langle\frac{1}{D\left(\beta_{i}\right) D\left(\beta_{j}\right)}\right\rangle_{\triangle_{2 N+2 S+2}^{(1)}}
\end{aligned}
$$

(ii) For any integers $N \geq 1$ and $S>1-N$, and finite sets of mutually distinct complex numbers 1.2 .3 such that $\beta \cap \mathbb{R}=\varnothing$, one has

$$
\left\langle\frac{\prod_{i=1}^{k} D^{2}\left(\alpha_{i}\right)}{\prod_{i=1}^{m} D^{2}\left(\beta_{i}\right)}\right\rangle_{\triangle_{N}^{(4)}}=\frac{C_{N+S}^{(4)}}{C_{N}^{(4)}} \frac{\prod(\alpha ; \beta)}{V(\alpha) V(\beta)} \operatorname{Pf}\left[W_{N}^{(4)}(\alpha, \beta \mid \alpha, \beta)\right]
$$

where $W_{N}^{(4)}$ is a skew-symmetric matrix with rows and columns parameterized by elements of $\alpha$ and $\beta$, and with matrix elements given by

$$
\begin{aligned}
& W_{N}^{(4)}\left(\alpha_{i}, \alpha_{j}\right)=\frac{C_{N+S-1}^{(4)}}{C_{N+S}^{(4)}}\left(\alpha_{i}-\alpha_{j}\right)\left\langle D^{2}\left(\alpha_{i}\right) D^{2}\left(\alpha_{j}\right)\right\rangle_{\triangle_{N+S-1}^{(4)}} \\
& W_{N}^{(4)}\left(\alpha_{i}, \beta_{j}\right)=\frac{1}{\alpha_{i}-\beta_{j}}\left\langle\frac{D^{2}\left(\alpha_{i}\right)}{D^{2}\left(\beta_{j}\right)}\right\rangle_{\triangle_{N+S}^{(4)}} \\
& W_{N}^{(4)}\left(\beta_{i}, \beta_{j}\right)=\frac{C_{N+S+1}^{(4)}}{C_{N+S}^{(4)}}\left(\beta_{i}-\beta_{j}\right)\left\langle\frac{1}{D^{2}\left(\beta_{i}\right) D^{2}\left(\beta_{j}\right)}\right\rangle_{\triangle_{N+S+1}^{(4)}}
\end{aligned}
$$

Note that the formulas for the orthogonal and symplectic ensembles above are essentially identical, except for the number of distinct eigenvalues, which in orthogonal averages is equal to twice that number in the symplectic averages, and for the form of the characteristic polynomial, which is equal to $D(\zeta)$ in the orthogonal case and to $D^{2}(\zeta)$ in the symplectic case.

The formulas in the theorem above require that the total number $k+m$ of factors in average (1.1.2) is even. These formulas can be easily extended to the situation when the total number of factors is odd. In order to do that, one needs to take the even case and send one of the parameters $\alpha_{i}, \beta_{j}$ to $\infty$. After the limit transition, some of the two-point averages in the matrix elements of $W_{N}^{(\beta)}$ will turn into one-point averages of the form $\langle\operatorname{det}(\zeta-H)\rangle,\langle 1 / \operatorname{det}(\zeta-H)\rangle$.

Interestingly enough, the analogous result in the unitary case looks more complicated! (Although, its proof is, actually, simpler.) A large part of the next result is contained in the previous work of Brezin-Hikami [15, 16], Strahov-Fyodorov [53], Baik, Deift and Strahov [5].

Theorem 1.2.2. (unitary case) For any integers $N \geq 1$ and $S>1-N$, and finite sets of mutually distinct complex numbers

$$
\begin{aligned}
\alpha^{-}=\left\{\alpha_{1}^{-}, \ldots, \alpha_{m_{1}}^{-}\right\}, & \alpha^{+}=\left\{\alpha_{1}^{+}, \ldots, \alpha_{k_{1}}^{-}\right\}, \\
\beta^{-}=\left\{\beta_{1}^{-}, \ldots, \beta_{m_{2}}^{-}\right\}, & \beta^{+}=\left\{\beta_{1}^{+}, \ldots, \beta_{k_{2}}^{+}\right\},
\end{aligned}
$$

with $\left|\alpha^{-}\right|-\left|\alpha^{+}\right|=\left|\beta^{-}\right|-\left|\beta^{+}\right|=S$, such that $\alpha^{+} \cap \mathbb{R}=\varnothing, \beta^{+} \cap \mathbb{R}=\varnothing$, one has

$$
\begin{aligned}
& \left\langle\frac{\prod_{i=1}^{m_{1}} D\left(\alpha_{i}^{-}\right) \prod_{i=1}^{m_{2}} D\left(\beta_{i}^{-}\right)}{\prod_{j=1}^{k_{1}} D\left(\alpha_{j}^{+}\right) \prod_{j=1}^{k_{2}} D\left(\beta_{j}^{+}\right)}\right\rangle_{\triangle_{N}^{(2)}}=(-1)^{\frac{\left(\left|\alpha^{-}\right|+\left|\beta^{-}\right|\right)^{2}+\left(\left|\beta^{-}\right|-\left|\alpha^{-}\right|\right)}{2}} \\
& \times \frac{C_{N+S}^{(2)}}{C_{N}^{(2)}} \frac{\prod\left(\alpha^{-} ; \alpha^{+}\right) \prod\left(\beta^{-} ; \beta^{+}\right)}{V\left(\alpha^{-}\right) V\left(\alpha^{+}\right) V\left(\beta^{-}\right) V\left(\beta^{+}\right)} \operatorname{det}\left[W_{N}^{(2)}\left(\alpha^{-}, \beta^{+} \mid \beta^{-}, \alpha^{+}\right)\right] .
\end{aligned}
$$


Here $W_{N}^{(2)}\left(\alpha^{-}, \beta^{+} \mid \beta^{-}, \alpha^{+}\right)$is a matrix with rows parameterized by elements of $\alpha^{-}$and $\beta^{+}$, columns parameterized by elements of $\beta^{-}$and $\alpha^{+}$, and with matrix elements

$$
\begin{aligned}
& W_{N}^{(2)}\left(\alpha_{i}^{-}, \beta_{j}^{-}\right)=\frac{C_{N+S-1}^{(2)}}{C_{N+S}^{(2)}}\left\langle D\left(\alpha_{i}^{-}\right) D\left(\beta_{j}^{-}\right)\right\rangle_{\triangle_{N+S-1}^{(2)}} \\
& W_{N}^{(2)}\left(\alpha_{i}^{-}, \alpha_{j}^{+}\right)=\frac{1}{\alpha_{i}^{-}-\alpha_{j}^{+}}\left\langle\frac{D\left(\alpha_{i}^{-}\right)}{D\left(\alpha_{j}^{+}\right)}\right\rangle_{\triangle_{N+S}^{(2)}} \\
& W_{N}^{(2)}\left(\beta_{i}^{+}, \beta_{j}^{-}\right)=\frac{1}{\beta_{i}^{+}-\beta_{j}^{-}}\left\langle\frac{D\left(\beta_{j}^{-}\right)}{D\left(\beta_{i}^{+}\right)}\right\rangle_{\triangle_{N+S}^{(2)}} \\
& W_{N}^{(2)}\left(\beta_{i}^{+}, \beta_{j}^{+}\right)=\frac{C_{N+S+1}^{(2)}}{C_{N+S}^{(2)}}\left\langle\frac{1}{D\left(\beta_{i}^{+}\right) D\left(\beta_{j}^{+}\right)}\right\rangle_{\triangle_{N+S+1}^{(2)}}
\end{aligned}
$$

Once again, the formula above holds for the even total number of determinants, but the odd case is easily obtained by sending one of the parameters $\alpha_{i}^{ \pm}, \beta_{j}^{ \pm}$to infinity.

It is worth noting that Theorem 1.2.2 provides many different expressions for the same averages, depending on how we split the factors in the numerator and denominator into groups. The resulting identities are often not easy to prove independently.

Define the $n$th correlation measure of $p_{N}^{(\beta)}$ by

$$
\rho_{n, N}^{(\beta)}\left(d x_{1}, \ldots, d x_{n}\right)=\frac{N !}{(N-n) !} \int_{x_{n+1}, x_{n+2}, \ldots, x_{N}} p_{N}^{(\beta)}\left(d x_{1}, \ldots, d x_{N}\right) .
$$

For a function $f(\zeta)$ of a complex variable $\zeta$, which is continuous in both half-planes $\operatorname{Im} \zeta>0$ and $\operatorname{Im} \zeta<0$ up to the real axis, we will denote by $[f(\zeta)]_{\zeta=x}, x \in \mathbb{R}$, the difference of the limit values of $f(\zeta)$ as $\zeta \rightarrow x$ from bottom and from top divided by $2 \pi i$ :

$$
[f(\zeta)]_{x}=\frac{1}{2 \pi i}(f(x-i 0)-f(x+i 0)) .
$$

The next statement is an easy corollary of Theorems 1.2 .2 and 1.2.1

Corollary 1.2.3. Take any $n \geq 1$ and assume that near $n$ points $x_{1}, \ldots, x_{n} \in \mathbb{R}$ the measure $\mu$ is absolutely continuous with respect to the Lebesgue measure, and its density their is uniformly Hölder continuous. Then the nth correlation measure of $\triangle_{N}^{(\beta)}$ (or $\triangle_{2 N}^{(\beta)}$ for $\beta=1$ ) has a continuous density near $\left(x_{1}, \ldots, x_{n}\right)$ which is given by

- For $\beta=2$

$$
\rho_{n, N}^{(2)}\left(x_{1}, \ldots, x_{n}\right)=\operatorname{det}\left[K_{N}^{(2)}\left(x_{i}, x_{j}\right)\right]_{i, j=1}^{n}
$$

where for $x \neq y$ the kernel is given by

$$
K^{(2)}(x, y)=\frac{1}{x-y}\left[\left\langle\frac{D(x)}{D(\zeta)}\right\rangle_{\triangle_{N}^{(2)}}\right]_{\zeta=y},
$$

and for $x=y$ the kernel is defined by continuity.

- For $\beta=1$

$$
\rho_{n, 2 N}^{(1)}\left(x_{1}, \ldots, x_{n}\right)=\operatorname{Pf}\left[K_{2 N}^{(1)}\left(x_{i}, x_{j}\right)\right]_{i, j=1}^{n}
$$

where the skew-symmetric $2 \times 2$ matrix kernel for $x \neq y$ is given by

$$
\left.K_{2 N}^{(1)}(x, y)=\left[\begin{array}{cc}
\frac{C_{2 N-2}^{(1)}}{C_{2 N}^{(1)}}(x-y)\langle D(x) D(y)\rangle_{\triangle_{2 N-2}^{(1)}} & \frac{1}{x-y}\left[\left\langle\frac{D(x)}{D(\zeta)}\right\rangle_{\triangle_{2 N}^{(1)}}\right]_{\zeta=y} \\
-\frac{1}{x-y}\left[\left\langle\frac{D(x)}{D(\zeta)}\right\rangle_{\triangle_{2 N}^{(1)}}\right]_{\zeta=y} & \frac{C_{2 N+2}^{(1)}}{C_{2 N}^{(1)}}(x-y)\left[\left\langle\frac{1}{D(\zeta) D(\eta)}\right\rangle_{\triangle_{2 N+2}^{(1)}}\right.
\end{array}\right]_{\zeta=x, \eta=y}\right]
$$


and for $x=y$ the kernel is defined by continuity.

- For $\beta=4$

$$
\rho_{n, N}^{(4)}\left(x_{1}, \ldots, x_{n}\right)=\operatorname{Pf}\left[K_{N}^{(4)}\left(x_{i}, x_{j}\right)\right]_{i, j=1}^{n}
$$

where the skew-symmetric $2 \times 2$ matrix kernel for $x \neq y$ is given by

$$
K_{N}^{(4)}(x, y)=\left[\begin{array}{cc}
\frac{C_{N-1}^{(4)}}{2 C_{N}^{(4)}}(x-y)\left\langle D^{2}(x) D^{2}(y)\right\rangle_{\triangle_{N+1}^{(4)}} & \frac{1}{2(x-y)}\left[\left\langle\frac{D^{2}(x)}{D^{2}(\zeta)}\right\rangle_{\triangle_{N}^{(4)}}\right]_{\zeta=y} \\
-\frac{1}{2(x-y)}\left[\left\langle\frac{D^{2}(x)}{D^{2}(\zeta)}\right\rangle_{\triangle_{N}^{(4)}}\right]_{\zeta=y} & \frac{C_{N+1}^{(4)}}{2 C_{N}^{(4)}}(x-y)\left[\left\langle\frac{1}{D^{2}(\zeta) D^{2}(\eta)}\right\rangle_{\triangle_{N-1}^{(4)}}\right]_{\zeta=x, \eta=y}
\end{array}\right]
$$

and for $x=y$ the kernel is defined by continuity.

If instead of asking for the measure $\mu$ to have nice density near $x_{1}, \ldots, x_{n}$, we require that $\mu$ is purely atomic near these points, then the formulas for the correlation functions above will continue to hold if we understand the symbol $[f(\zeta)]_{x}$ as the residue of the meromorphic function $f(\zeta)$ at the point $x$.

Using standard techniques of RMT, one can evaluate the two-point $(k+m=2)$ averages (1.1.2) via the (skew)-orthogonal polynomials associated with the problem, see Sections 2.7] 3.5] and 3.6 below. Then the formulas above yield a new proof of the well-known evaluation of the correlation functions of $p_{N}^{(\beta)}$ with $\beta=1,2,4$, in terms of (skew)-orthogonal polynomials (see e.g. [56]).

Let us emphasize that our approach provides a proof of the determinantal and pfaffian formulas for the correlation functions which does not use the (skew)-orthogonal polynomials.

1.3. The results: analytic part. Theorems 1.2.1 and 1.2.2 are very convenient for computing the limits of averages (1.1.2) as the size of matrices goes to infinity. In what follows we will use the notation $G O E_{N}, G U E_{N}, G S E_{N}$ to denote the measures $p_{N}^{(\beta)}$ with

$$
\beta=1, \mu(d x)=e^{-\frac{x^{2}}{2}} d x, \quad \beta=2, \mu(d x)=e^{-x^{2}} d x, \quad \beta=4, \mu(d x)=e^{-x^{2}} d x,
$$

respectively. The normalization is chosen in such a way that the needed scaling will be the same in all three cases. Note that in the case of Gaussian weights, the constants $C_{N}^{(\beta)}$ are explicitly computed as certain products of $\Gamma$-functions, see e.g. Ref. 44]. The values relevant for our three cases will be given in Section [5.

Theorem 1.3.1. (i) With the notation and assumptions of Theorem 1.2.1)(i), we have

$$
\begin{array}{r}
\lim _{N \rightarrow \infty} \frac{C_{2 N}^{(1)}}{C_{2 N+2 S}^{(1)}}(2 N)^{\frac{k m}{2}-\frac{k(k+1)}{4}-\frac{m(m-1)}{4}}\left\langle\frac{\prod_{i=1}^{k} D\left(\alpha_{i} / \sqrt{2 N}\right)}{\prod_{i=1}^{m} D\left(\beta_{i} / \sqrt{2 N}\right)}\right\rangle_{G O E(2 N)} \\
=\frac{\prod(\alpha ; \beta)}{V(\alpha) V(\beta)} \operatorname{Pf}\left[\mathbb{S}_{G O E}(\alpha, \beta \mid \alpha, \beta)\right]
\end{array}
$$

where $\mathbb{S}_{G O E}(\alpha, \beta \mid \alpha, \beta)$ is a skew-symmetric $(k+m) \times(k+m)$ matrix with rows and columns parameterized by elements of $\alpha$ and $\beta$, and with matrix elements given by

$$
\begin{aligned}
& \mathbb{S}_{G O E}\left(\alpha_{p}, \alpha_{q}\right)=-\frac{1}{\pi} \frac{\partial}{\partial \alpha_{i}} \frac{\sin \left(\alpha_{p}-\alpha_{q}\right)}{\alpha_{p}-\alpha_{q}}, \\
& \mathbb{S}_{G O E}\left(\alpha_{p}, \beta_{q}\right)= \begin{cases}-\frac{\exp i\left(\beta_{q}-\alpha_{p}\right)}{\beta_{q}-\alpha_{p}}, & \operatorname{Im} \beta_{q}>0, \\
\frac{\exp i\left(\alpha_{p}-\beta_{q}\right)}{\alpha_{p}-\beta_{q}}, & \operatorname{Im} \beta_{q}<0,\end{cases} \\
& \mathbb{S}_{G O E}\left(\beta_{p}, \beta_{q}\right)=2 \pi i \begin{cases}\int_{1}^{+\infty} \frac{\exp \left(i\left(\beta_{p}-\beta_{q}\right) t\right)}{t} d t, & \operatorname{Im} \beta_{p}>0, \operatorname{Im} \beta_{q}<0, \\
-\int_{1}^{+\infty} \frac{\exp \left(i\left(\beta_{q}-\beta_{p}\right) t\right)}{t} d t, & \operatorname{Im} \beta_{p}<0, \operatorname{Im} \beta_{q}>0, \\
0, & \text { in all other cases. }\end{cases}
\end{aligned}
$$


(ii) With the notation and assumptions of Theorem 1.2.1)(ii), we have

$$
\begin{array}{r}
\lim _{N \rightarrow \infty} \frac{C_{N}^{(4)}}{C_{N+S}^{(4)}}(2 N)^{\frac{k m}{2}-\frac{k(k-1)}{4}-\frac{m(m+1)}{4}}\left\langle\frac{\prod_{i=1}^{k} D^{2}\left(\alpha_{i} / \sqrt{2 N}\right)}{\prod_{i=1}^{m} D^{2}\left(\beta_{i} / \sqrt{2 N}\right)}\right\rangle_{G S E(N)} \\
=\frac{\prod(\alpha ; \beta)}{V(\alpha) V(\beta)} \operatorname{Pf}\left[\mathbb{S}_{G S E}(\alpha, \beta \mid \alpha, \beta)\right]
\end{array}
$$

where $\mathbb{S}_{G S E}(\alpha, \beta \mid \alpha, \beta)$ is a skew-symmetric $(k+m) \times(k+m)$ matrix with rows and columns parameterized by elements of $\alpha$ and $\beta$, and with matrix elements given by

$$
\begin{aligned}
& \mathbb{S}_{G S E}\left(\alpha_{p}, \alpha_{q}\right)=\frac{1}{\pi} \int_{0}^{1} \frac{\sin \left(\alpha_{p}-\alpha_{q}\right) t}{t} d t, \\
& \mathbb{S}_{G S E}\left(\alpha_{p}, \beta_{q}\right)= \begin{cases}-\frac{\exp i\left(\beta_{q}-\alpha_{p}\right)}{\beta_{q}-\alpha_{p}}, & \operatorname{Im} \beta_{q}>0, \\
\frac{\exp i\left(\alpha_{p}-\beta_{q}\right)}{\alpha_{p}-\beta_{q}}, & \operatorname{Im} \beta_{q}<0,\end{cases} \\
& \mathbb{S}_{G S E}\left(\beta_{p}, \beta_{q}\right)=2 \pi i \begin{cases}\frac{\partial}{\partial \beta_{p}} \frac{\exp i\left(\beta_{p}-\beta_{q}\right)}{\beta_{p}-\beta_{q}}, & \operatorname{Im} \beta_{p}>0, \operatorname{Im} \beta_{q}<0, \\
-\frac{\partial}{\partial \beta_{q}} \frac{\exp i\left(\beta_{q}-\beta_{p}\right)}{\beta_{q}-\beta_{p}}, & \operatorname{Im} \beta_{p}<0, \operatorname{Im} \beta_{q}>0, \\
0, & \text { in all other cases. }\end{cases}
\end{aligned}
$$

Similarly, Theorem 1.2 .2 yields

Theorem 1.3.2. With the notation and assumptions of Theorem 1.2 .2 we have

$$
\begin{aligned}
& \lim _{N \rightarrow \infty} \frac{C_{N}^{(2)}}{C_{N+S}^{(2)}}(2 N)^{-\frac{S^{2}}{2}}\left\langle\frac{\prod_{i=1}^{k_{1}} D\left(\alpha_{i}^{-} / \sqrt{2 N}\right) \prod_{i=1}^{k_{2}} D\left(\beta_{i}^{-} / \sqrt{2 N}\right)}{\prod_{j=1}^{m_{1}} D\left(\alpha_{j}^{+} / \sqrt{2 N}\right) \prod_{j=1}^{m_{2}} D\left(\beta_{j}^{+} / \sqrt{2 N}\right)}\right\rangle_{G U E(N)}= \\
& (-1)^{\frac{\left(\left|\beta^{-}\right|+\left|\alpha^{-}\right|\right)^{2}+\left(\left|\beta^{-}\right|-\left|\alpha^{-}\right|\right)}{2}} \frac{\prod\left(\alpha^{-} ; \alpha^{+}\right) \prod\left(\beta^{-} ; \beta^{+}\right)}{V\left(\alpha^{-}\right) V\left(\alpha^{+}\right) V\left(\beta^{-}\right) V\left(\beta^{+}\right)} \operatorname{det}\left[\mathbb{S}_{G U E}\left(\alpha^{-}, \beta^{+} \mid \beta^{-}, \alpha^{+}\right)\right]
\end{aligned}
$$

Here $\mathbb{S}_{G U E}\left(\alpha^{-}, \beta^{+} \mid \beta^{-}, \alpha^{+}\right)$is a matrix with rows parameterized by elements of $\alpha^{-}$and $\beta^{+}$, columns parameterized by elements of $\beta^{-}$and $\alpha^{+}$, and with matrix elements

$$
\begin{aligned}
& \mathbb{S}_{G U E}\left(\alpha_{p}^{-}, \beta_{q}^{-}\right)=\frac{1}{\pi} \frac{\sin \left(\alpha_{p}^{-}-\beta_{q}^{-}\right)}{\alpha_{p}^{-}-\beta_{q}^{-}}, \\
& \mathbb{S}_{G U E}\left(\alpha_{p}^{-}, \alpha_{q}^{+}\right)= \begin{cases}-\frac{\exp i\left(\alpha_{q}^{+}-\alpha_{p}^{-}\right)}{\alpha_{q}^{+}-\alpha_{p}^{-}}, & \operatorname{Im} \alpha_{q}^{+}>0, \\
\frac{\exp i\left(\alpha_{p}^{-}-\alpha_{q}^{+}\right)}{\alpha_{p}^{-}-\alpha_{q}^{+}}, & \operatorname{Im} \alpha_{q}^{+}<0,\end{cases} \\
& \mathbb{S}_{G U E}\left(\beta_{p}^{+}, \beta_{q}^{-}\right)= \begin{cases}\frac{\exp i\left(\beta_{p}^{+}-\beta_{q}^{-}\right)}{\beta_{p}^{+}-\beta_{q}^{-}}, & \operatorname{Im} \beta_{p}^{+}>0, \\
-\frac{\exp i\left(\beta_{q}^{-}-\beta_{p}^{+}\right)}{\beta_{q}^{-}-\beta_{p}^{+}}, & \operatorname{Im} \beta_{p}^{+}<0,\end{cases} \\
& \mathbb{S}_{G U E}\left(\beta_{p}^{+}, \alpha_{q}^{+}\right)=2 \pi i \begin{cases}\frac{\exp i\left(\beta_{p}^{+}-\alpha_{q}^{+}\right)}{\beta_{p}^{+}-\alpha_{q}^{+}}, & \operatorname{Im} \beta_{p}^{+}>0, \operatorname{Im} \alpha_{q}^{+}<0, \\
-\frac{\exp i\left(\alpha_{q}^{+}-\beta_{p}^{+}\right)}{\alpha_{q}^{+}-\beta_{p}^{+}}, & \operatorname{Im} \beta_{p}^{+}<0, \operatorname{Im} \alpha_{q}^{+}>0, \\
0, & \text { in all other cases. }\end{cases}
\end{aligned}
$$

Observe that if in any of the three cases (GOE, GUE, GSE) the number of determinants in the numerator is the same as the number of factors in the denominator, then no prefactors of the form $C_{N_{1}}^{(\beta)} / C_{N_{2}}^{(\beta)}$ or of the form $2 N$ raised to a power are needed, the limit exists without any normalization. We believe that this is a deep fact, however, at the moment we do not have a conceptual explanation for it.

Another intriguing observation is that if we take the same number of determinants in the numerator and denominator, and the signs of the imaginary parts of all the arguments in the denominator 
are the same, the limit turns out to be the same in all three cases! More exactly,

$$
\begin{aligned}
\lim _{N \rightarrow \infty}\left\langle\frac{\operatorname{det}\left(\alpha_{1} / \sqrt{2 N}-H\right) \cdots \operatorname{det}\left(\alpha_{k} / \sqrt{2 N}-H\right)}{\operatorname{det}\left(\beta_{1} / \sqrt{2 N}-H\right) \cdots \operatorname{det}\left(\beta_{k} / \sqrt{2 N}-H\right)}\right\rangle_{G O E(2 N), G U E(N), G S E(N)} \\
=\exp \left[ \pm i \sum_{j=1}^{k}\left(\alpha_{j}-\beta_{j}\right)\right]
\end{aligned}
$$

where the positive sign inside the exponential corresponds to the case $\operatorname{Im} \beta_{1}, \ldots, \operatorname{Im} \beta_{k}<0$, and the negative sign corresponds to the case $\operatorname{Im} \beta_{1}, \ldots, \operatorname{Im} \beta_{k}>0$. Again, it would be nice to have a conceptual explanation for such an unexpected coincidence. In particular, one may ask if such a limit exists for arbitrary $\beta>0$, and if so then whether it depends on $\beta$.

It is worth noting that if one formally uses the asymptotics of Theorems 1.3.1 1.3.2 in Corollary 1.2.3 to compute the asymptotics of the correlation functions then one easily recovers the wellknown results for the bulk scaling limit of the correlation kernels, see e.g. Refs. [27, [44]. For details of this computation see Remark 5.1.8, Section 5.1 below.

An important feature of the bulk scaling limit in random matrix models is that the limiting values of the correlation kernels turn out to be universal: they depend on $\beta$ but do not depend on the potential $Q(x)$. This universality property has been verified in full generality for $\beta=2$ case in Refs. [24, [8], and in the middle of the spectrum for $\beta=1,4$ in Ref. [25]. We expect that the bulk scaling limits of averages (1.1.2 which we computed in Theorems 1.3.1, 1.3.2 in the middle of the spectrum for Gaussian potentials, are also universal in a similar sense. For $\beta=2$ this was proved in Ref. [53], and for $\beta=1,4$ the question remains open.

1.4. The method: discrete approximation. In recent years we have seen a lot of progress in understanding discrete probabilistic models of random matrix type which come from various domains of mathematics. One important observation that becomes clear from the point of view of those discrete models is that the measures $p_{N}^{(\beta)}$ with $\beta=1,2,4$, may be viewed as degenerate cases of more general objects, the so-called determinantal point processes in the unitary case and pfaffian point processes in the orthogonal and symplectic case.

Inside these more general classes of point processes one also finds discrete analogs of the measures $p_{N}^{(\beta)}$. In the unitary case, the discrete ensemble is different from the continuous one only by the fact that the support of the measure $\mu$ is discrete. In the orthogonal and symplectic cases the difference is more substantial.

Let $\mathfrak{X}$ be a finite subset of $\mathbb{R}$ with an even number of elements. The discrete analog of the measure $p_{2 N}^{(1)}$ is a measure on $2 N$-point subsets $X$ of $\mathfrak{X}$ which have the following property: for any $x \in X$ the number of points in $X$ that are smaller than $x$ has the same parity as the number of point in $\mathfrak{X}$ that are smaller than $x$. The weight of one such subset is given by the familiar formula, cf. 1.2.2.

$$
\operatorname{Prob}\left(x_{1}, \ldots, x_{2 N}\right)=\mathrm{const} \prod_{1 \leq i<j \leq 2 N}\left|x_{i}-x_{j}\right| \cdot \prod_{i=1}^{2 N} f\left(x_{i}\right),
$$

where $f$ is some positive weight function. We call this measure a discrete orthogonal ensemble.

A discrete symplectic ensemble is defined by the same formula (1.4.1), but the admissible subsets $X$ are different: with any point $x$ such subset must contain the immediate predecessor of $x$ in $\mathfrak{X}$.

It is not hard to see that if we take $\mathfrak{X}$ to be a lattice then in the limit when the step of this lattice goes to zero, the discrete orthogonal/symplectic ensembles turn into measures similar to $p_{2 N}^{(1)}$ and $p_{N}^{(4)}$. (Indeed, if the points of $X$ are split into pairs, and in each pair the points are infinitesimally close, then the Vandermonde determinant of size $|X|$ in (1.4.1) is asymptotically given by the fourth power of the Vandermonde determinant of size $|X| / 2$ in scaled locations of the pairs.) 
The discrete orthogonal and symplectic ensembles are related by the particle-hole involution: if $X$ is from a discrete orthogonal ensemble than $\widehat{X}=\mathfrak{X} \backslash X$ is from a discrete symplectic ensemble (with a different weight function and different number of particles though) and vice versa. The particle-hole involution of a discrete unitary ensemble (which is a measure of the form $p_{N}^{(2)}$ with discretely supported $\mu$ ) is again a discrete unitary ensemble. In the $\beta=2$ case the particle-hole involution has been used before, see Refs. 9], [12 (Section 5), 14, 39, 6].

The motivation for introducing these discrete objects comes from representation theory; in certain models these are just different ways of parameterizing partitions. If $\lambda=\left(\lambda_{1}, \lambda_{2}, \ldots\right)$ is a partition then $\left\{2 \lambda_{i}-i\right\}$ is an admissible point configuration for a discrete orthogonal ensemble, while $\left\{\lambda_{i}-2 i, \lambda_{i}-2 i+1\right\}$ is an admissible point configuration for a discrete symplectic ensemble. The convenience of coordinates $\left\{\lambda_{i}-\beta i / 2\right\}$ in the models involving Vandermonde determinant raised to the power $\beta$ can be observed, e.g., in Refs. [43, [13. The fact that discrete orthogonal and symplectic ensembles are dual to each other comes from two different ways of parameterizing partitions: using rows or columns of the corresponding Young diagram. We hope to give more details on this connection in a future publication.

In the paper we actually prove analogs of Theorems 1.2.1 and 1.2.2 for discrete orthogonal, unitary, and symplectic ensembles and then obtain Theorems 1.2.1 and 1.2.2 by limit transition. Note that two parts of Theorem 1.2.1 in the discrete situation are simply equivalent - they are obtained one from the other by the particle-hole involution. This explains the similarity of the formulas.

The proofs in the discrete case are obtained as follows. It turns out that if we apply the particlehole involution on a suitable part of the phase space $\mathfrak{X}$ then the orthogonal/symplectic and unitary ensembles turn into what we call $L$-ensembles. This is another subclass of pfaffian/determinantal point processes; the processes in this subclass are defined using a matrix of size $|\mathfrak{X}| \times|\mathfrak{X}|$ which is often denoted by $L$. The discrete analogs of Theorems 1.2.1 and 1.2.2 interpreted in the language of this matrix $L$ provide some relations between matrix elements of $L$ and its resolvent. These relations are then proved using standard linear algebraic facts.

We would like to emphasize that after the objects of interest are represented in terms of $L$ ensembles, the proofs become very simple. The nontrivial part of our approach is in constructing the discrete models which make the statements easy to prove.

1.5. The asymptotics. Despite the fact that Theorems 1.3.1 and 1.3.2 look like rather simpleminded corollaries of Theorems 1.2.1 and 1.2.2. rigorous proofs of the asymptotic formulas do require some efforts.

The first step of our computation consists of expressing the two-point averages - matrix elements of $W_{N}^{(\beta)}$ in Theorems 1.2.1 and 1.2.2 - in terms of associated (skew)-orthogonal polynomials. As is well known, in all three Gaussian ensembles the corresponding polynomials are expressible through classical Hermite polynomials, see e.g. 44, 27, 1, 47]. Also, certain summation formulas of Christoffel-Darboux type for all three case $\beta=1,2,4$ are known, see [47, [58. Using these formulas and applying some algebraic transformations (which are least simple in the symplectic case), we write the two-point averages as finite expressions involving Hermite polynomials, their derivatives, and integrals.

Further computations are easy to explain — we replace the Hermite polynomials of large degree by their asymptotic oscillatory behavior near the origin. However, sometimes we need to do that in double or triple integrals, and the resulting limit integrals are often only conditionally convergent. Such actions require accurate estimates of the error terms, and they constitute a large part of Section 5. In these estimates it is essential for us to have uniform asymptotics of the Hermite polynomials on the real line. The needed results follow from much more general results of [23, 24] ${ }^{2}$; we adopt their formulas to the Hermite case.

\footnotetext{
${ }^{2}$ The classical estimates proved in [55] turn to be to be insufficient for our purposes.
} 
1.6. Final remarks. To conclude the introduction we would like to suggest the following debatable point of view which we developed while working on the subject:

The averages of products and ratios of characteristic polynomials are more fundamental characteristics of random matrix models than the correlation functions.

This is especially visible in the $\beta=1,4$ cases. Here are some arguments to defend this thesis:

- The pfaffian/determinantal formulas for these averages are simpler than those for the correlation functions. The formulas express many-point averages in terms of one- and two-point ones, and they do not require the introduction of (skew)-orthogonal polynomials or Christoffel-Darboux type kernels.

- The formulas for the correlation functions are easily recovered from those for averages (1).

- Even though there is no clear probabilistic sense in taking the bulk scaling limit of such averages, it does exist after proper normalization, and it appears to be fairly universal. The limiting correlation kernels are easily obtained from these limits.

We hope to provide further arguments related to discrete probabilistic models arising in representation theory in future publications.

1.7. Acknowledgements. We are very grateful to Grigori Olshanski for numerous discussions of the $\beta=2$ case. This research was partially conducted during the period one of the authors (A.B.) served as a Clay Mathematics Institute Research Fellow.

\section{Determinantal point Ensembles}

2.1. Point configurations. Let $\mathfrak{X}$ be a finite set. We will denote by $X, Y, \ldots$ subsets of the set $\mathfrak{X}$ and we will call them "point configurations". Let $\mathfrak{X}$ have a fixed splitting into the union of two disjoint subsets ("positive" and "negative"),

$$
\mathfrak{X}=\mathfrak{X}_{-} \sqcup \mathfrak{X}_{+}
$$

Then any point configuration $X$ is a unit of two disjoint sets as well,

$$
\begin{gathered}
X=X_{-} \sqcup X_{+} \\
X_{-}=X \cap \mathfrak{X}_{-}, \quad X_{+}=X \cap \mathfrak{X}_{+}
\end{gathered}
$$

and we will say that the point configuration $X$ consists of positive particles (elements of $X_{+}$) and negative particles (elements of $X_{-}$). If a given configuration includes an equal number of positive and negative particles we will say that such a configuration is a balanced point configuration. Thus

$$
\left|X_{+}\right|=\left|X_{-}\right|
$$

if $X=\left(X_{-} \mid X_{+}\right)$is a balanced configuration. Here $|A|$ denotes the number of elements in the set $A$.

If $X$ is a point configuration of particles in $\mathfrak{X}$ its complement $\mathfrak{X} \backslash X$ in the set $\mathfrak{X}$ will be a point configuration as well. It is natural to refer to the point configuration $\mathfrak{X} \backslash X$ as the point configuration of holes. The set $(\mathfrak{X} \backslash X) \cap \mathfrak{X}_{-}$will be called the set of positive holes, and the set $(\mathfrak{X} \backslash X) \cap \mathfrak{X}_{+}$will be called the set of negative holes.

To any configuration $X$ of particles there will correspond (in many ways) a balanced point configuration $Z$, which consists of both particles and holes. Namely let $X=\left(X_{-} \mid X_{+}\right)$be the splitting of the point configuration $X$ into positive and negative particles, and assume that $\left|X_{-}\right|-$ $\left|X_{+}\right|=S \geq 0$. Then the corresponding balanced configuration can be constructed in accordance with the formulas:

$$
\begin{aligned}
Z=Z_{-} \sqcup Z_{+}, Z_{+} & =Z \cap\left(\mathfrak{X}_{+} \sqcup \mathfrak{X}_{0}\right), Z_{-}=Z \cap\left(\mathfrak{X}_{-} \backslash \mathfrak{X}_{0}\right) \\
Z_{-} & =X_{-} \backslash \mathfrak{X}_{0} \\
Z_{+} & =X_{+} \sqcup\left(\mathfrak{X}_{0} \backslash X\right)
\end{aligned}
$$

where $\mathfrak{X}_{0}$ is an arbitrary subset of the set $\mathfrak{X}_{-}$consisting of $S$ points. Fig. 1 shows the decomposition of the set $\mathfrak{X}$, where the set $\mathfrak{X}_{0}$ is chosen to be the right-hand subset of the set $\mathfrak{X}_{-}$. Fig. 2 
represents the unbalanced particle-particle configuration X, and Fig. 3 explains the construction of the balanced particle-hole configuration $Z$.

It is not hard to see that the point configuration $Z$ constructed in such a way is a balanced point configuration, i.e. $\left|Z_{+}\right|=\left|Z_{-}\right|$. Indeed if $\left|X_{+}\right|=d$ then $\left|X_{-}\right|=S+d$. Assume that the chosen subset $\mathfrak{X}_{0}$ includes $d_{1}$ negative particles of the configuration $X$. It means that $\mathfrak{X}_{0}$ includes $S-d_{1}$ positive holes. The set $Z_{+}$consists of these positive holes and positive particles of the point configuration $X$, thus $\left|Z_{+}\right|=S-d_{1}+d$. Since $Z_{-}$consists of the negative particles lying outside the set $\mathfrak{X}_{0},\left|Z_{-}\right|=S+d-d_{1}$. Therefore $\left|Z_{+}\right|=\left|Z_{-}\right|$, and the configuration $Z$ defined above is a balanced configuration.

We will say that the balanced configuration $Z$ is obtained from the configuration $X$ by the particle-hole involution on the set $\mathfrak{X}_{0}$.

2.2. Definition of $L$-ensembles. First we recall the notion of determinantal $L$-ensembles following Daley and Vere-Jones [21, Borodin and Olshanski [11, 12. A random point process on the space $\mathfrak{X}$ is defined by an introduction of a probability $\operatorname{Prob}_{L}(X)$ for each subset $X$ of $\mathfrak{X}$, so that

$$
\sum_{X \subset \mathfrak{X}} \operatorname{Prob}_{L}(X)=1, \quad \operatorname{Prob}_{L}(X) \geq 0
$$

Let $L$ be a $\mathfrak{X} \times \mathfrak{X}$ matrix whose rows and columns are parameterized by the points of $\mathfrak{X}$. We use the notation $A(\alpha \mid \beta)$ for a matrix $A$ and subsets $\alpha=\left(\alpha_{1}, \ldots, \alpha_{a}\right), \beta=\left(\beta_{1}, \ldots, \beta_{b}\right)$ of its rows and columns, to denote the submatrix $\left\|A_{\alpha_{i} \beta_{j}}\right\|$ of $\mathrm{A}$. Then to any subset $X$ (or for any point configuration) there will correspond the diagonal minor det $L(X \mid X)$ of the matrix $L$. Assume further that any such diagonal minor of $L$ is nonnegative.

Definition 2.2.1. A random point process living on the space of point configurations in $\mathfrak{X}$ is called an $L$-ensemble, if

$$
\operatorname{Prob}_{L}(X)=\frac{\operatorname{det} L(X \mid X)}{\operatorname{det}(1+L)} \text {. }
$$

It is clear that $\sum_{X \subset \mathfrak{X}} \operatorname{Prob}_{L}(X)=1$ as the normalization constant $\operatorname{det}(1+L)$ has the following well-known decomposition:

$$
\operatorname{det}(1+L)=\sum_{X \subset \mathfrak{X}} \operatorname{det} L(X \mid X) .
$$

According to the decomposition of the set $\mathfrak{X}$ into the union of positive and negative subspaces, $\mathfrak{X}=\mathfrak{X}_{-} \sqcup \mathfrak{X}_{+}$we write the matrix $L$ in the block form:

$$
L=\left[\begin{array}{ll}
L_{--} & L_{-+} \\
L_{+-} & L_{++}
\end{array}\right]
$$

Let $h$ be a real function defined on $\mathfrak{X}$. We are interested in $L$-ensembles for which the matrix $L$ is given by the formula

$$
L=\left[\begin{array}{cc}
0 & A \\
-A^{T} & 0
\end{array}\right], \quad A(x, y)=\frac{h(x) h(y)}{x-y} .
$$

In that case we use the formula for Cauchy determinants to rewrite the expression for the probability of a random point configuration as

$$
\operatorname{Prob}_{L}(X)=\left\{\begin{array}{cl}
\frac{1}{\operatorname{det}(1+L)} \frac{V^{2}\left(X_{+}\right) V^{2}\left(X_{-}\right)}{\prod^{2}\left(X_{-} ; X_{+}\right)} h^{2}(X), & \left|X_{+}\right|=\left|X_{-}\right| \\
0, & \text { otherwise }
\end{array}\right.
$$

where $\prod(A ; B) \equiv \prod_{i=1}^{k} \prod_{j=1}^{l}\left(a_{i}-b_{j}\right)$ for any two sets $A=\left(a_{1}, \ldots, a_{k}\right), B=\left(b_{1}, \ldots, b_{l}\right), V(X)$ is the Vandermonde determinant associated with the set $X$,

$$
V(X)=\prod_{1 \leq i<j \leq N}\left(x_{i}-x_{j}\right), \quad X=\left(x_{1}, \ldots, x_{N}\right)
$$


and $h(X)=\prod_{j=1}^{N} h\left(x_{j}\right)$

2.3. $\hat{L}$-ensemble. We have seen in the section above that an $L$-ensemble is completely determined by a splitting of the discrete set $\mathfrak{X}$ into positive and negative parts, and by a weight $h$ on $\mathfrak{X}$. With a different splitting of the set $\mathfrak{X}, \mathfrak{X}=\left(\hat{\mathfrak{X}}_{-} \mid \hat{\mathfrak{X}}_{+}\right)$and different weight $\hat{h}$ we will construct another ensemble, which we will call $\hat{L}$-ensemble.

Let $\hat{\mathfrak{X}}_{-}=\mathfrak{X}_{-} \backslash \mathfrak{X}_{0}$ and $\hat{\mathfrak{X}}_{+}=\mathfrak{X}_{0} \sqcup \mathfrak{X}_{+},\left|\mathfrak{X}_{0}\right|=S$. As we have already seen in Section 2.1] to the unbalanced configurations $X$ of particles

$$
X=\left(X_{-} \mid X_{+}\right),\left|X_{-}\right|-\left|X_{+}\right|=S
$$

with respect to the splitting $\mathfrak{X}=\left(\mathfrak{X}_{-} \mid \mathfrak{X}_{+}\right)$there corresponds the balanced hole-particle configuration $Z$,

$$
Z=\left(Z_{-} \mid Z_{+}\right),\left|Z_{-}\right|=\left|Z_{+}\right|
$$

with respect to the new splitting $\mathfrak{X}=\left(\hat{\mathfrak{X}}_{-} \mid \hat{\mathfrak{X}}_{+}\right)$. The point configurations $X$ and $Z$ are related by the hole-particle involution on the set $\mathfrak{X}_{0}$ as it is described in Section 2.1.

Consider an $\hat{L}$-ensemble on $\mathfrak{X}$ with the matrix $\hat{L}$ having the following block structure:

$$
L=\left[\begin{array}{cc}
0 & \hat{A} \\
-\hat{A}^{T} & 0
\end{array}\right], \quad \hat{A}(x, y)=\frac{\hat{h}(x) \hat{h}(y)}{x-y}
$$

Here the decomposition of the matrix $\hat{L}$ into the blocks corresponds to the new splitting $\mathfrak{X}=$ $\left(\hat{\mathfrak{X}}_{-} \mid \hat{\mathfrak{X}}_{+}\right)$of the discrete set $\mathfrak{X}$. As soon as the particle-hole configurations $Z$ are balanced with respect to this splitting we find

$$
\operatorname{Prob}_{\hat{L}}(Z)=\frac{1}{\operatorname{det}(1+\hat{L})} \hat{h}^{2}(Z) \frac{V^{2}\left(Z_{-}\right) V^{2}\left(Z_{+}\right)}{\prod^{2}\left(Z_{-} ; Z_{+}\right)}
$$

The balanced configurations $Z$ of particles and holes were constructed from the unbalanced configurations $X$ of particles, in which the number of negative particles is larger by $S$ then the number of positive particles. Clearly, it is possible to rewrite $\operatorname{Prob}_{\hat{L}}(Z)$ in terms of the configurations $X$. With a suitable choice of the weight $\hat{h}$ the expression for $\operatorname{Prob}_{\hat{L}}(Z)$ in terms of configurations $X$ takes the same form as the right-hand side of equation (2.2.3) (up to a normalization constant). Namely, introduce the new weight $\hat{h}$ in terms of the old weight $h$ according to the formula:

$$
\hat{h}(z)= \begin{cases}h(z) \prod_{y \in \mathfrak{X}_{0}}(z-y), & z \in \mathfrak{X}_{-} \backslash \mathfrak{X}_{0}, \\ \frac{1}{h(z) \prod_{y \in \mathfrak{X}_{0}, y \neq z}(z-y)}, & z \in \mathfrak{X}_{0}, \\ \frac{h(z)}{\prod_{y \in \mathfrak{X}_{0}}(z-y)}, & z \in \mathfrak{X}_{+} .\end{cases}
$$

Proposition 2.3.1. This choice of the weight $\hat{h}$ (equation (2.3.3)) gives

$$
\operatorname{Prob}_{\hat{L}}(Z)=\frac{1}{\operatorname{det}(1+\hat{L})} \frac{1}{V^{2}\left(\mathfrak{X}_{0}\right) h^{2}\left(\mathfrak{X}_{0}\right)} h^{2}(X) \frac{V^{2}\left(X_{-}\right) V^{2}\left(X_{+}\right)}{\prod^{2}\left(X_{-} ; X_{+}\right)} .
$$

Proof. In order to see that equation (2.3.4) is valid we rewrite the expression

$$
h^{2}(X) \frac{V^{2}\left(X_{-}\right) V^{2}\left(X_{+}\right)}{\prod^{2}\left(X_{-} ; X_{+}\right)}
$$

in terms of the balanced configuration $Z$ (the relation between the unbalanced configuration $X$ and the balanced configuration is shown in Tab. 1 . We will also use the notation $Z_{+}^{I, I I}$ introduced 
there). In particular we find

$$
\begin{aligned}
& \text { - } h^{2}(X)=h^{2}\left(Z_{-}\right) \cdot \frac{h^{2}\left(\mathfrak{X}_{0}\right)}{h^{2}\left(Z_{+}^{I}\right)} \cdot h^{2}\left(Z_{+}^{I I}\right) \\
& \text { - } V^{2}\left(X_{-}\right)=V^{2}\left(\mathfrak{X}_{0} \backslash Z_{+}^{I}\right) \cdot V^{2}\left(Z_{-}\right) \cdot \prod^{2}\left(Z_{-} ; \mathfrak{X}_{0} \backslash Z_{+}^{I}\right) \\
& \text { - } V^{2}\left(X_{+}\right)=\frac{V^{2}\left(Z_{+}\right)}{V^{2}\left(Z_{+}^{I}\right) \cdot \prod^{2}\left(Z_{+}^{I} ; Z_{+}^{I I}\right)} \\
& \text { - } \prod^{2}\left(X_{-} ; X_{+}\right)=\prod^{2}\left(Z_{-} ; Z_{+}^{I I}\right) \cdot \prod^{2}\left(\mathfrak{X}_{0} \backslash Z_{+}^{I} ; Z_{+}^{I I}\right)
\end{aligned}
$$

Thus

$$
\frac{V^{2}\left(X_{-}\right) V^{2}\left(X_{+}\right)}{\prod^{2}\left(X_{-} ; X_{+}\right)}=\frac{V^{2}\left(Z_{-}\right) V^{2}\left(Z_{+}\right)}{\prod^{2}\left(Z_{-} ; Z_{+}\right)} \frac{V^{2}\left(\mathfrak{X}_{0} \backslash Z_{+}^{I}\right) \cdot \prod^{2}\left(Z_{-} ; \mathfrak{X}_{0}\right)}{V^{2}\left(Z_{+}^{I}\right) \cdot \prod^{2}\left(Z_{+}^{I} ; Z_{+}^{I I}\right) \cdot \prod^{2}\left(\mathfrak{X}_{0} \backslash Z_{+}^{I} ; Z_{+}^{I I}\right)}
$$

We rewrite $V^{2}\left(\mathfrak{X}_{0} \backslash Z_{+}^{I}\right)$

$$
V^{2}\left(\mathfrak{X}_{0} \backslash Z_{+}^{I}\right)=\frac{V^{2}\left(\mathfrak{X}_{0}\right)}{V^{2}\left(Z_{+}^{I}\right) \cdot \prod^{2}\left(Z_{+}^{I} ; \mathfrak{X}_{0} \backslash Z_{+}^{I}\right)}
$$

and note that the resulting denominator equals

$$
\begin{aligned}
& V^{2}\left(Z_{+}^{I}\right) \cdot \prod^{2}\left(Z_{+}^{I} ; Z_{+}^{I I}\right) \cdot \prod^{2}\left(\mathfrak{X}_{0} \backslash Z_{+}^{I} ; Z_{+}^{I}\right) \cdot \prod^{2}\left(\mathfrak{X}_{0} \backslash Z_{+}^{I} ; Z_{+}^{I I}\right) \cdot V^{2}\left(Z_{+}^{I}\right) \\
& =V^{2}\left(Z_{+}^{I}\right) \cdot \prod^{2}\left(Z_{+}^{I I} ; \mathfrak{X}_{0}\right) \cdot \prod^{2}\left(\mathfrak{X}_{0} \backslash Z_{+}^{I} ; Z_{+}^{I}\right) \cdot V^{2}\left(Z_{+}^{I}\right) \\
& =\prod_{\substack{x, y \in Z_{+}^{I} \\
y<x}}(x-y)^{2} \cdot \prod_{\substack{x \in Z_{+}^{I} \\
y \in \mathfrak{X}_{0} \backslash Z_{+}^{I}}}(x-y)^{2} \cdot \prod_{\substack{x \in Z_{+}^{I I} \\
y \in \mathfrak{X}_{0}}}(x-y)^{2} \cdot \prod_{\substack{x, y \in Z_{+}^{I} \\
y>x}}(x-y)^{2}=\prod_{\substack{x \in Z_{+} \\
y \in \mathfrak{X}_{0}, y \neq x}}(x-y)^{2} .
\end{aligned}
$$

Introducing $\hat{h}(z)$ by equation (2.3.3) we see that equation (2.3.4) is equivalent to equation (2.3.2).

2.4. Correlation functions for $L$-ensembles and averages of characteristic polynomials. By correlation functions for $L$-ensembles $\varrho(X)$ we mean the probabilities that the random configurations include fixed sets $X$. Thus

$$
\varrho(X)=\sum_{Y \supseteq X} \operatorname{Prob}_{L}(Y)
$$

It is known that the correlation functions $\varrho(X)$ are expressed as symmetric minors of the correlation kernel $K=L(1+L)^{-1}$, i.e.

$$
\varrho(X)=\operatorname{det} K(X \mid X) .
$$

The proof of this fact can be found in the book by Daley and Vere-Jones 21] (Exercise 5.4.7), and in Borodin and Olshanski [1] (Proposition 2.1), Borodin, Okounkov and Olshanski [10] (Appendix). Equation (2.4.1) tell us that random processes associated with $L$-ensembles are discrete determinantal point processes (see Borodin and Olshanski [11, 12, for definitions. A comprehensive survey on determinantal point processes is given in Soshnikov [51]).

In this Section we express the minors of the matrix $K$ in terms of averages of "characteristic polynomials" $E(\alpha, X)$ associated with $L$-ensembles. These objects were first introduced in Borodin and Olshanski [13] and are constructed as follows. With the above notations, for any fixed set $\alpha$ and point configurations $X$ and $Z$ we set

$$
\begin{aligned}
E(\alpha, X)= & \frac{\prod\left(\alpha ; X_{+}\right)}{\prod\left(\alpha ; X_{-}\right)}, E(\alpha, Z)=\frac{\prod\left(\alpha ; Z_{+}\right)}{\prod\left(\alpha ; Z_{-}\right)}, \\
& E(\alpha, X)=\frac{E(\alpha, Z)}{\prod\left(\alpha ; \mathfrak{X}_{0}\right)} .
\end{aligned}
$$


In order to compute the minors of the matrix $K$ we need the following Lemma.

Lemma 2.4.1. Let $A, B$ be two balanced configurations on the set $\mathfrak{X}$,

$$
\left|A_{+}\right|=\left|A_{-}\right|=a,\left|B_{+}\right|=\left|B_{-}\right|=b
$$

Then

where

$$
\operatorname{det}\left[L\left(A_{-}, B_{+} \mid B_{-}, A_{+}\right)\right]=(-)^{w_{a b}} h(A) h(B) \frac{V\left(A_{-}\right) V\left(A_{+}\right)}{\prod\left(A_{-} ; A_{+}\right)} \frac{V\left(B_{-}\right) V\left(B_{+}\right)}{\prod\left(B_{-} ; B_{+}\right)}
$$

$$
w_{a b}=a b+b^{2}+\frac{a(a-1)}{2}+\frac{b(b-1)}{2}
$$

Proof. The formula above follows from the explicit structure of the matrix $L$ (equation (2.2.2)), from formulas for the Cauchy determinants (equation (6.1.1) ) and the determinants of the block matrices (equation (6.2.2)).

Introduce nonintersecting sets $\alpha^{ \pm}, \beta^{ \pm}$of complex numbers with nonequal elements,

$$
\begin{array}{ll}
\alpha^{+}=\left(\alpha_{1}^{+}, \ldots, \alpha_{k_{1}}^{+}\right), & \beta^{+}=\left(\beta_{1}^{+}, \ldots, \beta_{k_{2}}^{+}\right), \\
\alpha^{-}=\left(\alpha_{1}^{-}, \ldots, \alpha_{m_{1}}^{-}\right), & \beta^{-}=\left(\beta_{1}^{-}, \ldots, \beta_{m_{2}}^{-}\right) .
\end{array}
$$

Assume that $\alpha^{ \pm} \cap \mathfrak{X}=0, \beta^{ \pm} \cap \mathfrak{X}=0$, and

$$
\left|\alpha^{+}\right|-\left|\alpha^{-}\right|=\left|\beta^{+}\right|-\left|\beta^{-}\right|=S \text {. }
$$

In what follows we extend the definitions of the matrices $K, L$ to the sets $\alpha^{ \pm}, \beta^{ \pm}$in the following way. We add to $L$ rows parameterized by $\alpha^{-} \sqcup \beta^{+}$and columns parameterized by $\alpha^{+} \sqcup \beta^{-}$, and then define new matrix elements of $L$ according to (2.2.1)-(2.2.2), where we assume that $\alpha^{-}$and $\beta^{-}$are added to $\mathfrak{X}_{-}$, and $\alpha^{+}, \beta^{+}$are added to $\mathfrak{X}_{+}$. Then we set extended $K$ to be related to the extended $L$ by $K=L(1+L)^{-1}$.

Proposition 2.4.2. The minor $\operatorname{det}\left[K\left(\alpha^{-}, \beta^{+} \mid \beta^{-}, \alpha^{+}\right)\right]$can be given as a normalized average of a ratio of the functions $E(.,$.$) introduced above with respect to the \hat{L}$-ensemble. Namely,

$$
\begin{aligned}
\operatorname{det}\left[K\left(\alpha^{-}, \beta^{+} \mid \beta^{-}, \alpha^{+}\right)\right] & =(-)^{w_{\alpha, \beta}}\left[\frac{\operatorname{det}(1+\hat{L})}{\operatorname{det}(1+L)} h^{2}\left(\mathfrak{X}_{0}\right) V^{2}\left(\mathfrak{X}_{0}\right)\right] \\
& \times\left[h(\alpha) \frac{V\left(\alpha^{-}\right) V\left(\alpha^{+}\right)}{\prod\left(\alpha^{-} ; \alpha^{+}\right)}\right]\left[h(\beta) \frac{V\left(\beta^{-}\right) V\left(\beta^{+}\right)}{\prod\left(\beta^{-} ; \beta^{+}\right)}\right] \\
& \times\left[\frac{\prod\left(\alpha^{-} ; \mathfrak{X}_{0}\right) \prod\left(\beta^{-} ; \mathfrak{X}_{0}\right)}{\prod\left(\alpha^{+} ; \mathfrak{X}_{0}\right) \prod\left(\beta^{+} ; \mathfrak{X}_{0}\right)}\right]\left\langle\frac{E\left(\alpha^{+}, Z\right) E\left(\beta^{+}, Z\right)}{E\left(\alpha^{-}, Z\right) E\left(\beta^{-}, Z\right)}\right\rangle_{\hat{L}}
\end{aligned}
$$

where $w_{\alpha, \beta}=\frac{1}{2}\left[\left(\left|\alpha^{-}\right|+\left|\beta^{-}\right|\right)^{2}+\left|\beta^{-}\right|-\left|\alpha^{-}\right|\right]$.

Proof. Equation (6.5.2) gives

$$
\operatorname{det}\left[K\left(\alpha^{-}, \beta^{+} \mid \beta^{-}, \alpha^{+}\right)\right]=\sum_{X \subset \mathfrak{X}} \frac{\operatorname{det} L\left(\alpha^{-}, \beta^{+}, X \mid \beta^{-}, \alpha^{+}, X\right)}{\operatorname{det}(1+L)} .
$$

We define the following sets

$$
\begin{aligned}
& A_{+}=\alpha^{+} \cup X_{+}, \quad A_{-}=\alpha^{-} \cup X_{-}, \\
& B_{+}=\beta^{+} \cup X_{+}, \quad B_{-}=\beta^{-} \cup X_{-} .
\end{aligned}
$$

Only those configurations $X$ will contribute to the sum in equation (2.4.6) for which the sets $A_{-}$, $A_{+}$have equal number of elements and the sets $B_{-}, B_{+}$have equal number of elements. Otherwise

$$
\operatorname{det}\left[L\left(\alpha^{-}, \beta^{+}, X \mid \beta^{-}, \alpha^{+}, X\right)\right]=0 \text {. }
$$


It means that the sum in equation (2.4.6) runs over unbalanced configurations $X$,

$$
\left|X_{-}\right|-\left|X_{+}\right|=S
$$

Now we apply Lemma 2.4 .1 to compute $\operatorname{det}\left[L\left(\alpha^{-}, \beta^{+}, X \mid \beta^{-}, \alpha^{+}, X\right)\right]$ with $A_{ \pm}, B_{ \pm}$given by equations (2.4.7) and (2.4.8) respectively. We find

$$
\begin{aligned}
\operatorname{det}\left[K\left(\alpha^{-}, \beta^{+} \mid \beta^{-}, \alpha^{+}\right)\right] & =(-)^{w_{\alpha, \beta}}[\operatorname{det}(1+L)]^{-1} \\
& \times\left[h(\alpha) \frac{V\left(\alpha^{-}\right) V\left(\alpha^{+}\right)}{\prod\left(\alpha^{-} ; \alpha^{+}\right)}\right]\left[h(\beta) \frac{V\left(\beta^{-}\right) V\left(\beta^{+}\right)}{\prod\left(\beta^{-} ; \beta^{+}\right)}\right] \\
& \times \sum_{X}\left[\frac{E\left(\alpha^{+}, X\right) E\left(\beta^{+}, X\right)}{E\left(\alpha^{-}, X\right) E\left(\beta^{-}, X\right)}\right] h^{2}(X) \frac{V\left(X^{-}\right) V\left(X^{+}\right)}{\prod^{2}\left(X^{-} ; X^{+}\right)}
\end{aligned}
$$

Since the sum in this equation runs over unbalanced configurations, we cannot interpret the sum as an average over the $L$-ensemble. However, it is possible to rewrite this sum in terms of the balanced configurations $Z$ of particles and holes as it was explained in Section 2.3. Then equation (2.3.4) tell us that the sum in equation above is just an average over $\hat{L}$-ensemble. Replacing the sum over unbalanced configurations $X$ by the sum over balanced configurations $Z$ and using (2.4.2) we prove the Proposition.

Remark 2.4.3. The Proposition above clearly gives the matrix elements of the matrix $K$ in terms of averages of $E^{\prime s}$. For example,

$$
K\left(\beta_{i}^{+} \mid \alpha_{j}^{+}\right)=\frac{\operatorname{det}(1+\hat{L})}{\operatorname{det}(1+L)} h^{2}\left(x_{0}\right) \frac{h\left(\beta_{i}^{+}\right) h\left(\alpha_{j}^{+}\right)}{\left(\beta_{i}^{+}-x_{0}\right)\left(\alpha_{j}^{+}-x_{0}\right)}\left\langle E\left(\beta_{i}^{+}, Z\right) E\left(\alpha_{j}^{+}, Z\right)\right\rangle_{\hat{L}}
$$

where the set $\mathfrak{X}_{0}$ in the definition of the ensemble $\hat{L}$ (see Tab.1) consists of only one point $x_{0}$, $\mathfrak{X}_{0}=\left\{x_{0}\right\}$. The point $x_{0}$ is an arbitrary point in $\mathfrak{X}_{-}$. The weight $\hat{h}$ of this $\hat{L}$-ensemble is

$$
\hat{h}(z)= \begin{cases}h(z)\left(z-x_{0}\right), & z \in \mathfrak{X}_{-} \backslash\{x\}_{0} \\ \frac{1}{h\left(x_{0}\right)}, & z=x_{0} \\ \frac{h(z)}{\left(z-x_{0}\right)}, & z \in \mathfrak{X}_{+}\end{cases}
$$

as it follows from expression (2.3.3).

2.5. Discrete polynomial ensembles. Assume that a nonnegative function $f(x)$ is given on a finite set $\mathfrak{X} \subset \mathbb{R}$. We also require that $f$ does not vanish at least at $N$ distinct points. Then the monic orthogonal polynomials $\pi_{0}=1, \pi_{1}, \ldots, \pi_{N-1}$ can be introduced by orthogonalizing the system $\left(1, x, \ldots, x^{N-1}\right)$ in the Hilbert space $L^{2}(\mathfrak{X}, f \mu)$. Here $\mu$ denotes the counting measure on the set $\mathfrak{X}$. The orthogonality condition for the monic orthogonal polynomials on $\mathfrak{X}$ is

$$
\sum_{x \in \mathfrak{X}} \pi_{j}(x) \pi_{k}(x) f(x)=c_{j}^{2} \delta_{j k} .
$$

Let $\operatorname{Conf}_{N}(\mathfrak{X})$ denote the set of $N$-point configurations (subsets) in $\mathfrak{X}$. Such configurations will be denoted by $X^{\triangle}, X^{\triangle} \in \operatorname{Conf}_{N}(\mathfrak{X})$. Consider a point process on $\mathfrak{X}$ which lives on $\operatorname{Con} f_{N}(\mathfrak{X})$ and for which the probability of a configuration $X$ is given by

$$
\operatorname{Prob}\left(X^{\triangle}\right)=\mathrm{const} \prod_{x \in X^{\triangle}} f(x) \cdot V^{2}\left(X^{\triangle}\right), X^{\triangle} \in \operatorname{Conf}_{N}(\mathfrak{X}) .
$$

We will denote this process by $\triangle_{N}(f)$.

Definition 2.5.1. The point process $\triangle_{N}(f)$ will be called $N$-point $(\beta=2)$ discrete polynomial ensemble with the weight function $f$. (The value of $\beta$ refers to the power of $V(X)$ in (2.5.2).) 
For symmetric functions $g\left(X^{\triangle}\right)=g\left(x_{1}^{\triangle}, \ldots, x_{N}^{\triangle}\right)$ of points of the configuration $X^{\triangle}$, the average $\langle g\rangle_{\triangle_{N}(f)}$ with respect to $\operatorname{Prob}\left(X^{\triangle}\right)$ is

$$
\langle g\rangle_{\triangle_{N}(f)} \equiv \frac{\sum_{X^{\triangle} \in \operatorname{Conf}_{N}(\mathfrak{X})} g\left(X^{\triangle}\right) f\left(X^{\triangle}\right) \cdot V^{2}\left(X^{\triangle}\right)}{\sum_{X^{\triangle} \in \operatorname{Conf}_{N}(\mathfrak{X})} f\left(X^{\triangle}\right) \cdot V^{2}\left(X^{\triangle}\right)}
$$

Define the characteristic polynomial $d(\xi)$ associated with the point configuration $X^{\triangle}$ and the complex parameter $\xi$ :

$$
d(\xi)=\prod\left(\xi ; X^{\triangle}\right)
$$

Then the discrete variant of the classical Heine identity is given by the following

Proposition 2.5.2.

$$
\pi_{N}(\xi)=\langle d(\xi)\rangle_{\triangle_{N}(f)}
$$

where $\langle\cdot\rangle_{\triangle_{N}(f)}$ denotes the average over the discrete polynomial ensemble $\triangle_{N}(f)$.

Proof. The proof of this relation is an application of standard arguments of the theory of orthogonal polynomials (see, for example, Deift [22], Chapter 3).

Proposition 2.5.3. The m-point correlation function

$$
\varrho_{m}\left(y_{1}, \ldots, y_{m}\right)=\sum_{\substack{X^{\triangle} \in \operatorname{Conf}_{N}(\mathfrak{X}) \\ X^{\triangle} \ni\left\{y_{1}, \ldots, y_{m}\right\}}} \operatorname{Prob}\left(X^{\triangle}\right)
$$

for the discrete polynomial ensemble is given by the determinantal formula

$$
\varrho_{m}\left(y_{1}, \ldots, y_{m}\right)=\operatorname{det}\left[K_{N}^{C D}\left(y_{i}, y_{j}\right)\right]_{i, j=1}^{m}, m=1,2, \ldots
$$

where $K_{N}^{C D}(x, y)$ stands for the normalized Christoffel-Darboux kernel,

$$
K_{N}^{C D}(x, y)=\sqrt{f(x) f(y)} \sum_{j=0}^{N-1} \frac{\pi_{j}(x) \pi_{j}(y)}{c_{j}^{2}} .
$$

One possible proof of this proposition is to adopt standard arguments of the random matrix theory to the case of the discrete ensemble $\triangle_{N}(f)$ (see, for example, Mehta [44], Deift [22]). In the following sections we present another proof. First, we will show that the discrete polynomial ensembles are equivalent to the $L$-ensembles (see Section 2.6). Specifically, the discrete polynomial ensembles and the $L$-ensembles can be transformed to each other by a suitable particle-hole involution. Second, we compute the averages of products and ratios of characteristic polynomials $E(\alpha, X)$ for the $L$-ensembles (see Section [2.7). From these averages we deduce the averages of products and ratios of characteristic polynomials $d(\xi)$ for the discrete orthogonal ensemble $\triangle_{N}(f)$. Third, we show in Section 2.8 how formula (2.5.4) can be obtained from the resulting expressions.

2.6. Equivalence of $L$-ensembles and discrete polynomial ensembles. The connection between the discrete polynomial ensembles $\triangle_{N}(f)$ and $L$-ensembles was demonstrated in Borodin and Olshanski [12, $\$ 5$. In the terminology of Section 2.1. this relation is a consequence of the particle-hole involution on an $N$-point subset of $\mathfrak{X}$. To be more specific, assume that the subset $\mathfrak{X}_{+}$in the definition of the $L$-ensemble (see Section 2.2) is finite, and consists of $N$ points. Thus $\left|\mathfrak{X}_{+}\right|=N$. We assume that the weight $h$ of the $L$-ensemble is nonnegative on $\mathfrak{X}$ and strictly positive on $\mathfrak{X}_{+}$. If the $L$-ensemble is defined by equation (2.2.2) then only balanced configurations $X$ with $\left|X_{+}\right|=\left|X_{-}\right|$have non-zero probabilities. Consider the particle-hole configuration $X^{\triangle}$ constructed by the particle-hole involution on the set $\mathfrak{X}_{+}$. Thus

$$
X^{\triangle}=X_{-} \sqcup\left(\mathfrak{X}_{+} \backslash X_{+}\right) .
$$


Clearly, the configuration $X^{\triangle}$ consists of precisely $N-\left|X_{+}\right|+\left|X_{-}\right|=N$ points where $N-\left|X_{+}\right|$is the number of negative holes, and $\left|X_{-}\right|$is the number of negative particles. Moreover, it is always possible to introduce a weight $f$ on $\mathfrak{X}$ in such a way that $\operatorname{Prob}\left(X^{\triangle}\right)$ takes the same form as in the definition of the discrete polynomial ensemble (equation (2.5.2)). Namely, introduce the weight $f$ in terms of the weight $h$ of the $L$-ensemble by the formula

$$
f(x)= \begin{cases}\frac{h^{2}(x)}{\prod_{y \in \mathfrak{X}_{+}}(x-y)^{2}}, & x \in \mathfrak{X}_{-}, \\ \frac{1}{h^{2}(x) \prod_{y \in \mathfrak{X}_{+}, y \neq x}(x-y)^{2}}, & x \in \mathfrak{X}_{+} .\end{cases}
$$

Proposition 2.6.1. The discrete polynomial ensemble with the weight $f$ and the $L$-ensemble defined by equation (2.2.2)-(2.2.3) are connected by the particle-hole involution on the set $\mathfrak{X}_{+}$.

Proof. See Borodin and Olshanski [12], Proposition 5.2.

Once the $L$-ensemble is given, we can construct an $\hat{L}$-ensemble with respect to the new splitting of the set $\mathfrak{X}, \mathfrak{X}=\left(\hat{\mathfrak{X}}_{-} \mid \hat{\mathfrak{X}}_{+}\right)$as in Section 2.3 (see also Tab. 1). Clearly $\left|\hat{\mathfrak{X}}_{+}\right|=N+S$. This $\hat{L}_{-}$ ensemble induces a discrete $(N+S)$-point polynomial ensemble under the particle-hole involution on the set $\hat{\mathfrak{X}}_{+}$. It follows from Proposition 2.6.1 that the induced polynomial ensemble with the weight $\hat{f}(x)$ will be equivalent to the $\hat{L}$-ensemble with the weight $\hat{h}(x)$ if

$$
\hat{f}(x)= \begin{cases}\frac{\hat{h}^{2}(x)}{\prod_{y \in \hat{\mathfrak{X}}_{+}}(x-y)^{2}}, & x \in \hat{\mathfrak{X}}_{-} \\ \frac{1}{\hat{h}^{2}(x) \prod_{y \in \hat{\mathfrak{X}}_{+}, y \neq x}(x-y)^{2}}, & x \in \hat{\mathfrak{X}}_{+}\end{cases}
$$

Denote by $\triangle_{N}(f)$ the discrete $N$-point polynomial ensemble which is equivalent to the $L$-ensemble, and by $\hat{\triangle}_{N+S}(\hat{f})$ the discrete $(N+S)$-point polynomial ensemble which is equivalent to the $\hat{L}$ ensemble.

Proposition 2.6.2. The ensembles $\triangle_{N}(f)$ and $\hat{\triangle}_{N+S}(\hat{f})$ have the same weight,

$$
\hat{f}(x)=f(x), \quad x \in \mathfrak{X} .
$$

Proof. We use formula (2.3.3) to express the weight $\hat{h}(x)$ in terms of the weight $h(x)$ in expression (2.6.2), and after that formula (2.6.1) which expresses the weight $f(x)$ in terms of the weight $h(x)$ :

- $x \in \mathfrak{X}_{-} \backslash \mathfrak{X}_{0}$

- $x \in \mathfrak{X}_{0}$

$$
\begin{aligned}
\hat{f}(x)=\frac{\hat{h}^{2}(x)}{\prod_{y \in \hat{\mathfrak{X}}_{+}}(x-y)^{2}} & =\frac{h^{2}(x) \prod_{y \in \mathfrak{X}_{0}}(x-y)^{2}}{\prod_{y \in \hat{\mathfrak{X}}_{+}}(x-y)^{2}} \\
& =\frac{h^{2}(x)}{\prod_{y \in \mathfrak{X}_{+}}(x-y)^{2}}=f(x)
\end{aligned}
$$

$$
\begin{aligned}
\hat{f}(x)=\frac{1}{\hat{h}^{2}(x) \prod_{y \in \hat{\mathfrak{X}}_{+}, y \neq x}(x-y)^{2}} & =\frac{h^{2}(x) \prod_{y \in \mathfrak{X}_{0}, y \neq x}(x-y)^{2}}{\prod_{y \in \hat{\mathfrak{X}}_{+}, y \neq x}(x-y)^{2}} \\
& =\frac{h^{2}(x)}{\prod_{y \in \mathfrak{X}_{+}}(x-y)^{2}}=f(x)
\end{aligned}
$$

- $x \in \mathfrak{X}_{+}$

$$
\begin{array}{r}
\hat{f}(x)=\frac{1}{\hat{h}^{2}(x) \prod_{y \in \hat{\mathfrak{X}}_{+}, y \neq x}(x-y)^{2}}=\frac{\prod_{y \in \mathfrak{X}_{0}}(x-y)^{2}}{h^{2}(x) \prod_{y \in \hat{\mathfrak{X}}_{+}, y \neq x}(x-y)^{2}} \\
=\frac{1}{h^{2}(x) \prod_{y \in \mathfrak{X}_{+}, y \neq x}(x-y)^{2}}=f(x)
\end{array}
$$


The correspondence between the $L$-ensembles and the discrete orthogonal polynomial ensembles is summarized in Tab. 2.

2.7. Averages of characteristic polynomials. The goal of this Section is to compute the averages of characteristic polynomials for the discrete polynomial ensembles. We will use the equivalence of the $L$-ensembles and the discrete polynomial ensembles, and deduce from Proposition 2.4 .2 the averages of products and ratios of characteristic polynomials. Specifically, we will express the right-hand side of equation (2.4.5) in terms of averages and normalization constants of the ensemble $\triangle_{N}(f)$. This ensemble has the weight $f$ given by equation (2.6.1). Thus $\triangle_{N}(f)$ is connected with the $L$-ensemble by the particle-hole involution on the set $\mathfrak{X}_{+}$in accordance with Proposition 2.6.1]

We begin with the constant in equation (2.4.5). Denote by $C_{N}$ and $C_{N+S}$ the normalization constants for $\triangle_{N}(f)$ and $\triangle_{N+S}(f)$ :

$$
\begin{aligned}
C_{N} & =\sum_{X \in \operatorname{Conf}_{N}(\mathfrak{X})} V^{2}(X) \cdot f(X), \\
C_{N+S} & =\sum_{X \in \operatorname{Conf}_{N+S}(\mathfrak{X})} V^{2}(X) \cdot f(X) .
\end{aligned}
$$

Proposition 2.7.1. The constant in equation 2.4.5) is equal to the ratio of the normalization constants $C_{N}$ and $C_{N+S}$, i.e.

$$
\frac{C_{N+S}}{C_{N}}=V^{2}\left(\mathfrak{X}_{0}\right) h^{2}\left(\mathfrak{X}_{0}\right) \frac{\operatorname{det}(1+\hat{L})}{\operatorname{det}(1+L)} .
$$

Proof. The expression $[\operatorname{det}(1+L)]^{-1}$ represents the probability that a random configuration $X$ of the $L$-ensemble is empty, i.e.

$$
\operatorname{Prob}(X=\emptyset)=[\operatorname{det}(1+L)]^{-1}
$$

The empty configuration (of particles) for the $L$-ensemble corresponds to the configuration $X^{\triangle}=$ $\mathfrak{X}_{+}$of the $N$ positive holes for the $\triangle_{N}(f)$ ensemble. Since the $L$-ensemble is equivalent to the $\triangle_{N}(f)$ ensemble we have

$$
\operatorname{Prob}(X=\emptyset)=\operatorname{Prob}\left(X^{\triangle}=\mathfrak{X}_{+}\right)=\frac{1}{C_{N}} V^{2}\left(\mathfrak{X}_{+}\right) f\left(\mathfrak{X}_{+}\right) .
$$

Therefore

$$
[\operatorname{det}(1+L)]^{-1}=\frac{1}{C_{N}} V^{2}\left(\mathfrak{X}_{+}\right) f\left(\mathfrak{X}_{+}\right) .
$$

We repeat the above considerations for the $\hat{L}$-ensemble and conclude that

$$
\begin{aligned}
\frac{\operatorname{det}(1+\hat{L})}{\operatorname{det}(1+L)}=\frac{C_{N+S}}{C_{N}} \frac{V^{2}\left(\mathfrak{X}_{+}\right) f\left(\mathfrak{X}_{+}\right)}{V^{2}\left(\mathfrak{X}_{0} \sqcup \mathfrak{X}_{+}\right) f\left(\mathfrak{X}_{0} \sqcup \mathfrak{X}_{+}\right)} \\
=\frac{C_{N+S}}{C_{N}} \frac{V^{2}\left(\mathfrak{X}_{0}\right) \prod^{2}\left(\mathfrak{X}_{0} ; \mathfrak{X}_{+}\right) f\left(\mathfrak{X}_{0}\right)}{V_{N}} \frac{1}{V^{2}\left(\mathfrak{X}_{0}\right) h^{2}\left(\mathfrak{X}_{0}\right)} . \\
=\frac{C_{N+S}}{C_{n}}
\end{aligned}
$$

Here we have used formula (2.6.1) to obtain the last equation.

Let the sets $\alpha^{ \pm}, \beta^{ \pm}$be defined as in Section 2.4. Recall that $d(\xi)$ was defined in equation (2.5.3).

Theorem 2.7.2. For any integer $N \geq 1$, take an integer $S$ such that $N-|\mathfrak{X}|+1 \leq S \leq N-1$, complex numbers $\alpha^{-}=\left(\alpha_{1}^{-}, \ldots, \alpha_{m_{1}}^{-}\right), \alpha^{+}=\left(\alpha_{1}^{+}, \ldots, \alpha_{k_{1}}^{+}\right), \beta^{-}=\left(\beta_{1}^{-}, \ldots, \beta_{m_{2}}^{-}\right), \beta^{+}=\left(\beta_{1}^{+}, \ldots, \beta_{k_{2}}^{+}\right)$ such that $\left|\alpha^{-}\right|-\left|\alpha^{+}\right|=\left|\beta^{-}\right|-\left|\beta^{+}\right|=S$, in each set $\alpha^{ \pm}, \beta^{ \pm}$the numbers are pairwise distinct, 
and the sets $\alpha^{+}, \beta^{+}$do not intersect $\mathfrak{X}$. Then the average of products and ratios of characteristic polynomials with respect to the discrete polynomial ensemble is given by the formula

$$
\begin{aligned}
& \left\langle\frac{\prod_{i=1}^{m_{1}} d\left(\alpha_{i}^{-}\right) \prod_{i=1}^{m_{2}} d\left(\beta_{i}^{-}\right)}{\prod_{j=1}^{k_{1}} d\left(\alpha_{j}^{+}\right) \prod_{j=1}^{k_{2}} d\left(\beta_{j}^{+}\right)}\right\rangle_{\triangle_{N}(f)}=\left[\frac{C_{N-S}}{C_{N}}\right] \\
& \times(-)^{w_{\alpha \beta}} \frac{\prod\left(\alpha^{-} ; \alpha^{+}\right) \prod\left(\beta^{-} ; \beta^{+}\right)}{V\left(\alpha^{-}\right) V\left(\alpha^{+}\right) V\left(\beta^{-}\right) V\left(\beta^{+}\right)} \operatorname{det}\left[W_{N}\left(\alpha^{-}, \beta^{+} \mid \beta^{-}, \alpha^{+}\right)\right]
\end{aligned}
$$

where the kernel function $W_{N}$ is defined by

- $W_{N}\left(\alpha_{i}^{-}, \beta_{j}^{-}\right)=\frac{C_{N-S-1}}{C_{N-S}}\left\langle d\left(\alpha_{i}^{-}\right) d\left(\beta_{j}^{-}\right)\right\rangle_{\triangle_{N-S-1}(f)}$

- $W_{N}\left(\alpha_{i}^{-}, \alpha_{j}^{+}\right)=\frac{1}{\alpha_{i}^{-}-\alpha_{j}^{+}}\left\langle\frac{d\left(\alpha_{i}^{-}\right)}{d\left(\alpha_{j}^{+}\right)}\right\rangle_{\triangle_{N-S}(f)}$

- $W_{N}\left(\beta_{i}^{+}, \beta_{j}^{-}\right)=\frac{1}{\beta_{i}^{+}-\beta_{j}^{-}}\left\langle\frac{d\left(\beta_{j}^{-}\right)}{d\left(\beta_{i}^{+}\right)}\right\rangle_{\triangle_{N-S}(f)}$

- $W_{N}\left(\beta_{i}^{+}, \alpha_{j}^{+}\right)=\frac{C_{N-S+1}}{C_{N-S}}\left\langle\frac{1}{d\left(\beta_{i}^{+}\right) d\left(\alpha_{j}^{+}\right)}\right\rangle_{\triangle_{N-S+1}(f)}$

and $S, w_{\alpha \beta}$ are expressed in terms of $\left|\alpha^{ \pm}\right|,\left|\beta^{ \pm}\right|$as in Proposition 2.4.2. Note that $S$ above can be any integer, positive or negative.

Proof. Assume first that $S \geq 0$. Then the proof is based on the expression for the minors of the $K$ matrix in terms of averages of $E^{\prime s}$ over the $\hat{L}$-ensemble (see Proposition 2.4.2). The equivalence of the $L$-ensembles and orthogonal polynomial ensembles enables us to rewrite averages of $E^{\prime s}$ as averages over characteristic polynomials with respect to orthogonal polynomial ensembles. We note that

$$
E(\alpha, Z) \equiv \frac{\prod\left(\alpha ; Z^{+}\right)}{\prod\left(\alpha ; Z^{-}\right)}=\frac{\prod\left(\alpha ; \mathfrak{X}_{0}\right) \prod\left(\alpha ; \mathfrak{X}_{+}\right)}{\prod\left(\alpha ; Z^{\triangle}\right)}
$$

where $Z^{\triangle}$ is a random point configuration for the ensemble $\hat{\triangle}_{N+S}(f)$. We insert the expression above for $E(\alpha, Z)$ to the formula for the minors of the matrix $K$ in Proposition 2.4.2. According to Proposition 2.6.1 and Proposition 2.6.2 the average in the formula for det $\left[K\left(\alpha^{-}, \beta^{+} \mid \beta^{-}, \alpha^{+}\right)\right]$ can be understood as that over the discrete polynomial ensemble $\triangle_{N+S}(f)$. Thus the formula for the minors of the matrix $K$ takes the form

$$
\begin{aligned}
\operatorname{det}\left[K\left(\alpha^{-}, \beta^{+} \mid \beta^{-}, \alpha^{+}\right)\right] & =(-)^{w_{\alpha, \beta}}\left[\frac{C_{N+S}}{C_{N}}\right]\left[h(\alpha) \frac{V\left(\alpha^{-}\right) V\left(\alpha^{+}\right)}{\prod\left(\alpha^{-} ; \alpha^{+}\right)}\right]\left[h(\beta) \frac{V\left(\beta^{-}\right) V\left(\beta^{+}\right)}{\prod\left(\beta^{-} ; \beta^{+}\right)}\right] \\
& \times \frac{\prod\left(\alpha^{+} ; \mathfrak{X}_{+}\right) \prod\left(\beta^{+} ; \mathfrak{X}_{+}\right)}{\prod\left(\alpha^{-} ; \mathfrak{X}_{+}\right) \prod\left(\beta^{-} ; \mathfrak{X}_{+}\right)}\left\langle\frac{\prod_{i=1}^{m_{1}} d\left(\alpha_{i}^{-}\right) \prod_{i=1}^{m_{2}} d\left(\beta_{i}^{-}\right)}{\prod_{j=1}^{k_{1}} d\left(\alpha_{j}^{+}\right) \prod_{j=1}^{k_{2}} d\left(\beta_{j}^{+}\right)}\right\rangle_{\triangle_{N+S}(f)} .
\end{aligned}
$$

It is clear that the formula above expresses the matrix elements of the matrix $K$ as averages of corresponding products and ratios of characteristic polynomials. In particular we find

$$
\begin{aligned}
K\left(\beta_{i}^{+} \mid \alpha_{j}^{+}\right) & =h\left(\alpha_{j}^{+}\right) h\left(\beta_{i}^{+}\right) \prod\left(\alpha_{j}^{+} ; \mathfrak{X}_{+}\right) \prod\left(\beta_{i}^{+} ; \mathfrak{X}_{+}\right) \\
& \times \frac{C_{N+1}}{C_{N}}\left\langle\frac{1}{d\left(\beta_{i}^{+}\right) d\left(\alpha_{j}^{+}\right)}\right\rangle_{\triangle_{N+1}(f)}, \\
K\left(\alpha_{i}^{-} \mid \beta_{j}^{-}\right) & =\frac{C_{N-1}}{C_{N}} h\left(\alpha_{i}^{-}\right) h\left(\beta_{j}^{-}\right) \prod^{-1}\left(\alpha_{i}^{-} ; \mathfrak{X}_{+}\right) \prod^{-1}\left(\beta_{j}^{-} ; \mathfrak{X}_{+}\right) \\
& \times\left\langle d\left(\alpha_{i}^{-}\right) d\left(\beta_{j}^{-}\right)\right\rangle_{\triangle_{N-1}(f)},
\end{aligned}
$$




$$
\begin{aligned}
K\left(\alpha_{i}^{-} \mid \alpha_{j}^{+}\right) & =h\left(\alpha_{i}^{-}\right) h\left(\alpha_{j}^{+}\right) \prod^{-1}\left(\alpha_{i}^{-} ; \mathfrak{X}_{+}\right) \prod\left(\alpha_{j}^{+} ; \mathfrak{X}_{+}\right) \\
& \times \frac{1}{\alpha_{i}^{-}-\alpha_{j}^{+}}\left\langle\frac{d\left(\alpha_{i}^{-}\right)}{d\left(\alpha_{j}^{+}\right)}\right\rangle_{\triangle_{N}(f)}, \\
K\left(\beta_{i}^{+} \mid \beta_{j}^{-}\right) & =h\left(\beta_{i}^{+}\right) h\left(\beta_{j}^{-}\right) \prod\left(\beta_{i}^{+} ; \mathfrak{X}_{+}\right) \prod^{-1}\left(\beta_{j}^{-} ; \mathfrak{X}_{+}\right) \\
& \times \frac{1}{\beta_{i}^{+}-\beta_{j}^{-}}\left\langle\frac{d\left(\beta_{j}^{-}\right)}{d\left(\beta_{i}^{+}\right)}\right\rangle_{\triangle_{N}(f)} .
\end{aligned}
$$

Expressing the elements of the matrix $K$ in the left-hand side of equation (2.7.2) in accordance with these formulas we prove the Theorem after some simplifications.

If $S<0$ we can prove Proposition 2.4.2 and Theorem 2.7.2 in a similar way considering $\mathfrak{X}_{0}$ in the construction of balanced particle-hole configurations as a subset of $\mathfrak{X}_{+}$. Another possibility to see that formula (2.7.1) for $S<0$ holds is to perform the particle-hole involution on the whole $\mathfrak{X}$. Under this particle-hole involution formula (2.7.1) does note change. However, $S>0$ is transformed to $S<0$.

Remark 2.7.3. In formula (2.7.1) the number of characteristic polynomials is greater or smaller than that in the numerator by $2|S|$. The case when these numbers are different by an odd integer is obtained from (2.7.1) by taking the limit $\alpha_{i} \rightarrow \infty$ or $\beta_{j} \rightarrow \infty$ for some $i$ or $j$, and using relation $d(\xi)=\xi^{N}(1+\mathcal{O}(1)), \xi \rightarrow \infty$.

Remark 2.7.4. Theorem 2.7.2 combines the different formulas of two-point function type obtained in Brezin and Hikami [16, Strahov and Fyodorov [53] into one formula (equation (2.7.1)).

Proposition 2.7.5. The kernels $W_{N}$ in the theorem above can be expressed in terms of the monic discrete orthogonal polynomials $\pi_{k}(x)$ and their Cauchy type transforms $h_{k}(\epsilon)$,

$$
h_{k}(\epsilon)=\frac{1}{2 \pi i} \sum_{x \in \mathfrak{X}} \frac{\pi_{k}(x) f(x)}{x-\epsilon}
$$

In particular we find

$$
\begin{gathered}
W_{N}\left(\alpha_{i}^{-}, \beta_{j}^{-}\right)=\frac{C_{N-S-1}}{C_{N-S}} \frac{\pi_{N-S}\left(\alpha_{i}^{-}\right) \pi_{N-S-1}\left(\beta_{j}^{-}\right)-\pi_{N-S}\left(\beta_{j}^{-}\right) \pi_{N-S-1}\left(\alpha_{i}^{-}\right)}{\alpha_{i}^{-}-\beta_{j}^{-}} \\
W_{N}\left(\alpha_{i}^{-}, \alpha_{k}^{+}\right)=\gamma_{N-S-1} \frac{\pi_{N-S}\left(\alpha_{k}^{+}\right) h_{N-S-1}\left(\alpha_{i}^{-}\right)-\pi_{N-S-1}\left(\alpha_{k}^{+}\right) h_{N-S}\left(\alpha_{i}^{-}\right)}{\alpha_{i}^{-}-\alpha_{k}^{+}} \\
W_{N}\left(\beta_{i}^{+}, \beta_{j}^{-}\right)=\gamma_{N-S-1} \frac{\pi_{N-S}\left(\beta_{i}^{+}\right) h_{N-S-1}\left(\beta_{j}^{-}\right)-\pi_{N-S-1}\left(\beta_{i}^{+}\right) h_{N-S}\left(\beta_{j}^{-}\right)}{\beta_{i}^{+}-\beta_{j}^{-}} \\
W_{N}\left(\beta_{i}^{+}, \beta_{j}^{-}\right)=\frac{C_{N-S+1}}{C_{N}} \gamma_{N-S} \gamma_{N-S-1} \frac{h_{N-S}\left(\beta_{i}^{+}\right) h_{N-S-1}\left(\beta_{j}^{+}\right)-h_{N-S}\left(\beta_{j}^{+}\right) h_{N-S-1}\left(\beta_{i}^{+}\right)}{\beta_{i}^{+}-\beta_{j}^{+}}
\end{gathered}
$$

Here we have introduced the coefficients $\gamma_{k}$,

$$
\gamma_{k}=-\frac{2 \pi i}{c_{k}^{2}}
$$

Proof. The computations for the case of the discrete orthogonal ensemble $\triangle_{N}(f)$ are similar to that for the case of the ensemble of Hermitian matrices (see Strahov and Fyodorov [53], Baik, Deift and Strahov [5]). 
2.8. Correlation functions of discrete polynomial ensemble. This Section shows the relation between $m$-point correlation function $\varrho_{m}\left(y_{1}, \ldots, y_{m}\right)$ for the discrete polynomial ensembles and averages of characteristic polynomials. The Proposition below gives $\varrho_{m}\left(y_{1}, \ldots, y_{m}\right)$ in terms of these averages.

Proposition 2.8.1. For any $y_{1}, \ldots, y_{m} \in \mathfrak{X}$ we have

$$
\varrho_{m}\left(y_{1}, \ldots, y_{m}\right)=\operatorname{Res}_{v_{1}=y_{1}} \ldots \operatorname{Res}_{v_{m}=y_{m}}\left[\left[\frac{\partial^{m}}{\partial u_{1} \ldots \partial u_{m}}\left\langle\frac{d\left(u_{1}\right) \ldots d\left(u_{m}\right)}{d\left(v_{1}\right) \ldots d\left(v_{m}\right)}\right\rangle_{\triangle_{N}(f)}\right]_{u=v}\right]
$$

Proof. In order to see that that the expression for $\varrho_{m}\left(y_{1}, \ldots, y_{m}\right)$ is correct we differentiate the product of characteristic polynomials in the numerator,

$$
\frac{\partial^{m}}{\partial u_{1} \ldots \partial u_{m}}\left[\prod_{i=1}^{m} \prod_{j=1}^{N}\left[u_{i}-x_{j}^{\triangle}\right]\right]=\sum_{j_{1}, \ldots, j_{m}=1}^{N}\left[\frac{1}{\prod_{k=1}^{m}\left(u_{k}-x_{j_{k}}^{\triangle}\right)}\right] \prod_{i=1}^{m} \prod_{j=1}^{N}\left[u_{i}-x_{j}^{\triangle}\right] .
$$

Thus the formula in the proposition is equivalent to the following one:

$$
\varrho_{m}\left(y_{1}, \ldots, y_{m}\right)=\operatorname{Res}_{v_{1}=y_{1}} \ldots \operatorname{Res}_{v_{m}=y_{m}}\left[\sum_{X \in C o n f_{N}(\mathfrak{X})} \operatorname{Prob}(X)\left[\sum_{j_{1}, \ldots, j_{m}=1}^{N} \frac{1}{\prod_{k=1}^{m}\left(v_{k}-x_{j_{k}}^{\triangle}\right)}\right]\right],
$$

which is evidently equivalent to the definition of $\varrho_{m}$.

Remark 2.8.2. The above argument applies to any point process on $\mathfrak{X}$, not just $\triangle_{N}(f)$.

Proposition 2.8.3. The m-point correlation function of the discrete ensemble $\triangle_{N}(f)$ is given by the formula

$$
\varrho_{m}\left(y_{1}, \ldots, y_{m}\right)=\operatorname{det}\left[K\left(y_{i}, y_{j}\right)\right]_{i, j=1}^{m}
$$

where

$$
K(x, y)= \begin{cases}\operatorname{Res}_{\xi=y}\left[\frac{1}{x-\xi}\left\langle\frac{d(x)}{d(\xi)}\right\rangle_{\triangle_{N}(f)}\right], & x \neq y \\ \operatorname{Res}_{\xi=y}\left[\left.\frac{d}{d \eta}\left\langle\frac{d(\eta)}{d(\xi)}\right\rangle\right|_{\eta=\xi}\right], & x=y .\end{cases}
$$

Proof. We note first that if we take

$$
\left|\beta^{+}\right|=\left|\beta^{-}\right|=0, \quad\left|\alpha^{+}\right|=\left|\alpha^{-}\right|=k
$$

in Theorem 2.7.2 the formula for averages of characteristic polynomials takes the following form:

$$
\left\langle\operatorname{det}\left[\frac{1}{u_{i}-v_{j}} \frac{d\left(u_{i}\right)}{d\left(v_{j}\right)}\right]_{i, j=1}^{k}\right\rangle_{\triangle_{N}(f)}=\operatorname{det}\left[\frac{1}{u_{i}-v_{j}}\left\langle\frac{d\left(u_{i}\right)}{d\left(v_{j}\right)}\right\rangle_{\triangle_{N}(f)}\right]_{i, j=1}^{k}
$$

We multiply the right-hand and left-hand sides of the equation above by the product $\prod_{j=1}^{k}\left(u_{j}-v_{j}\right)$. After that we differentiate $k$ times with respect to $u_{1}, \ldots u_{k}$, take $u_{1}=v_{1}, \ldots, u_{k}=v_{k}$ and find 
(after simple manipulations)

$$
\begin{aligned}
& \left\langle\left|\begin{array}{cccc}
G\left(v_{1}\right) & \frac{1}{v_{1}-v_{2}} & \cdots & \frac{1}{v_{1}-v_{k}} \\
\frac{1}{v_{2}-v_{1}} & G\left(v_{2}\right) & \cdots & \frac{1}{v_{2}-v_{k}} \\
\vdots & & & \\
\frac{1}{v_{k}-v_{1}} & \frac{1}{v_{k}-v_{2}} & \cdots & G\left(v_{k}\right)
\end{array}\right|_{\triangle_{N}(f)}=\right. \\
& \left|\begin{array}{cccc}
\left\langle G\left(v_{1}\right)\right\rangle_{\triangle_{N}(f)} & \frac{1}{v_{1}-v_{2}}\left\langle\frac{d\left(v_{1}\right)}{d\left(v_{2}\right)}\right\rangle_{\triangle_{N}(f)} & \cdots & \frac{1}{v_{1}-v_{k}}\left\langle\frac{d\left(v_{1}\right)}{d\left(v_{k}\right)}\right\rangle_{\triangle_{N}(f)} \\
\frac{1}{v_{2}-v_{1}}\left\langle\frac{d\left(v_{2}\right)}{d\left(v_{1}\right)}\right\rangle_{\triangle_{N}(f)} & \left\langle G\left(v_{2}\right)\right\rangle_{\triangle_{N}(f)} & \cdots & \frac{1}{v_{2}-v_{k}}\left\langle\frac{d\left(v_{2}\right)}{d\left(v_{k}\right)}\right\rangle_{\triangle_{N}(f)} \\
\vdots & \frac{1}{v_{k}-v_{2}}\left\langle\frac{d\left(v_{k}\right)}{d\left(v_{2}\right)}\right\rangle_{\triangle_{N}(f)} & \cdots & \left\langle G\left(v_{k}\right)\right\rangle_{\triangle_{N}(f)}
\end{array}\right|
\end{aligned}
$$

where we have introduced

$$
G(v)=\sum_{j=1}^{N} \frac{1}{v-x_{j}}
$$

We note

$$
\begin{aligned}
\underset{v_{1}=y_{1}}{\operatorname{Res}} & \ldots \operatorname{ves}_{v_{m}=y_{m}}^{\operatorname{Res}}\left[\left\langle G\left(v_{1}\right) \ldots G\left(v_{k}\right)\right\rangle_{\triangle_{N}(f)}\right]= \\
& =\sum_{X \in \operatorname{Con}_{N}(\mathfrak{X})}\left[\sum_{j_{1}, \ldots, j_{m}=1}^{N} \delta_{y_{1}, x_{j_{1}}} \ldots \delta_{y_{m}, x_{j_{m}}}\right] \operatorname{Prob}(X) \\
& =\varrho_{m}\left(y_{1}, \ldots, y_{m}\right)
\end{aligned}
$$

Thus the residue of the left-hand side of equation (2.8.5) is precisely the $m$-point correlation function. Now we compute the residue of the right-hand side of equation (2.8.5). It follows from the definition of $K(x, y)$ (see equation (2.8.3) ) that

$$
\operatorname{Res}_{v=y}\left[\langle G(v)\rangle_{\triangle_{N}(f)}\right]=K(y, y)
$$

The determinant in the right-hand side of equation (2.8.5) is a sum of products, and the off-diagonal elements are included as the cyclic products. The residues of the cyclic products are then the cyclic products of the kernels $K\left(y_{i}, y_{j}\right)$, for example

$$
\begin{gathered}
\underset{v_{1}=y_{1}}{\operatorname{Res}} \ldots \operatorname{Res}_{v_{j}=y_{j}}\left[\frac{1}{v_{1}-v_{2}} \frac{1}{v_{2}-v_{3}} \cdots \frac{1}{v_{j}-v_{1}}\left\langle\frac{d\left(v_{1}\right)}{d\left(v_{2}\right)}\right\rangle_{\triangle_{N}(f)}\left\langle\frac{d\left(v_{2}\right)}{d\left(v_{3}\right)}\right\rangle_{\triangle_{N}(f)} \ldots\left\langle\frac{d\left(v_{j}\right)}{d\left(v_{1}\right)}\right\rangle_{\triangle_{N}(f)}\right] \\
=K\left(y_{1}, y_{2}\right) K\left(y_{2}, y_{3}\right) \ldots K\left(y_{j}, y_{1}\right)
\end{gathered}
$$

It means that the residues of the right-hand side of equation (2.8.5) is det $\left[K\left(y_{i}, y_{j}\right)\right]_{i, j=1}^{m}$ which proves the Proposition.

2.9. Proof of Proposition 2.5.3. This standard result (see Mehta [4], Deift [22]) follows from the formula for the averages of two characteristic polynomials [34, 53, 5],

$$
\frac{1}{x-\xi}\left\langle\frac{d(x)}{d(\xi)}\right\rangle_{\triangle_{N}(f)}=\gamma_{N-1} \frac{\pi_{N}(x) h_{N-1}(\xi)-\pi_{N-1}(x) h_{N}(\xi)}{x-\xi}
$$


Indeed,

Therefore Proposition 2.8 .3 gives

$$
\operatorname{Res}_{\epsilon=y}\left[h_{k}(\epsilon)\right]=-\frac{1}{2 \pi i} f(y) \pi_{k}(y)
$$

$$
K(x, y)=\frac{1}{c_{N-1}^{2}} f(y) \frac{\pi_{N}(x) \pi_{N-1}(y)-\pi_{N-1}(x) \pi_{N}(y)}{x-y}
$$

when $x \neq y$, and

$$
\begin{aligned}
K(x, x) & =\frac{1}{c_{N-1}^{2}} f(x)\left[\pi_{N}^{\prime}(x) \pi_{N-1}(x)-\pi_{N-1}^{\prime}(x) \pi_{N}(x)\right] \\
& =f(x) \sum_{j=0}^{N-1} \frac{\pi_{j}(x) \pi_{j}(x)}{c_{j}^{2}}
\end{aligned}
$$

when $x=y$. Taking into account the Christoffel-Darboux summation formula (see [55] ) we see that the determinant with the kernel $K(x, y)$ is equal to the determinant with the Christoffel-Darboux kernel $K_{N}^{C D}(x, y)$.

\section{Pfaffian point ensembles}

3.1. Pfaffian $L$-ensembles. Given a set $\mathfrak{X}$ let us construct two copies of $\mathfrak{X}$, and denote them by $\mathfrak{X}^{\prime}$ and $\mathfrak{X}^{\prime \prime}$. We then introduce a $2 \times 2$ matrix valued function $L(x, y)$, which depends on two variables $x, y \in \mathfrak{X}$

$$
L(x, y)=\left[\begin{array}{ll}
L\left(x^{\prime}, y^{\prime}\right) & L\left(x^{\prime}, y^{\prime \prime}\right) \\
L\left(x^{\prime \prime}, y^{\prime}\right) & L\left(x^{\prime \prime}, y^{\prime \prime}\right)
\end{array}\right]
$$

Here we denote by the same letter $L 2 \times 2$ matrix valued function of two arguments $x, y \in \mathfrak{X}$, and the scalar function whose arguments are taken from $\mathfrak{X}^{\prime} \sqcup \mathfrak{X}^{\prime \prime}$. Once $x, y$ take values in $\mathfrak{X}$, the variables $x^{\prime}, y^{\prime}\left(x^{\prime \prime}, y^{\prime \prime}\right)$ are the elements of $\mathfrak{X}^{\prime}\left(\mathfrak{X}^{\prime \prime}\right)$ corresponding to $x, y$. Assume that this function $L$ is antisymmetric. Then $L(x, y)$ defines a $2 \times 2$ block antisymmetric matrix on $\mathfrak{X}$.

To any $X \subset \mathfrak{X}$ there will correspond a $2 \times 2$ block antisymmetric submatrix of $L$. We denote this submatrix by $L(X \mid X)$. If $X$ consists of $m$ points,

$$
X=\left(x_{1}, \ldots, x_{m}\right), \quad X \subset \mathfrak{X}
$$

the submatrix $L(X \mid X)$ has the form

$$
L(X \mid X)=\left[\begin{array}{ccccc}
0 & L\left(x_{1}^{\prime}, x_{1}^{\prime \prime}\right) & \cdots & L\left(x_{1}^{\prime}, x_{m}^{\prime}\right) & L\left(x_{1}^{\prime}, x_{m}^{\prime \prime}\right) \\
-L\left(x_{1}^{\prime}, x_{1}^{\prime \prime}\right) & 0 & & L\left(x_{1}^{\prime \prime}, x_{m}^{\prime}\right) & L\left(x_{1}^{\prime \prime}, x_{m}^{\prime \prime}\right) \\
\vdots & & & & \\
-L\left(x_{1}^{\prime}, x_{m}^{\prime}\right) & -L\left(x_{1}^{\prime \prime}, x_{m}^{\prime}\right) & & 0 & L\left(x_{m}^{\prime}, x_{m}^{\prime \prime}\right) \\
-L\left(x_{1}^{\prime}, x_{m}^{\prime \prime}\right) & -L\left(x_{1}^{\prime \prime}, x_{m}^{\prime \prime}\right) & & -L\left(x_{m}^{\prime}, x_{m}^{\prime \prime}\right) & 0
\end{array}\right]
$$

Denote by Pf $A$ the Pfaffian of an even dimensional antisymmetric matrix $A$. The definition of $\operatorname{Pf} A$ is given in Appendix, Section 6.6. Assume that the matrix $L$ has the property

$$
\operatorname{Pf} L(X \mid X) \geq 0, \quad \forall X \subset \mathfrak{X} .
$$

Let $J$ be $2 \times 2$ block matrix of format $\mathfrak{X} \times \mathfrak{X}$ with matrix elements

$$
J(x, y)= \begin{cases}{\left[\begin{array}{cc}
0 & 1 \\
-1 & 0
\end{array}\right],} & x=y \\
0, & \text { otherwise }\end{cases}
$$

Definition 3.1.1. A point process on $\mathfrak{X}$ defined by

$$
\operatorname{Prob}_{L}(X)=\frac{\operatorname{Pf} L(X \mid X)}{\operatorname{Pf}(J+L)}, \quad \forall X \subset \mathfrak{X}
$$

is called the Pfaffian $L$-ensemble. 
The fact that $\sum_{X \subset \mathfrak{X}} \operatorname{Prob}_{L}(X)=1$ follows from the expansion of $\operatorname{Pf}(J+L)$ into a sum of Pfaffians of the symmetric $2 \times 2$ block submatrices $L(X \mid X)$ of $L$, i. e.

$$
\operatorname{Pf}(J+L)=\sum_{X \subset \mathfrak{X}} \operatorname{Pf} L(X \mid X) .
$$

The striking property of the Pfaffian $L$-ensembles is that the $m$-point correlation function $\varrho_{m}(Y)$ is given by a Pfaffian,

$$
\varrho_{m}(Y)=\operatorname{Pf}\left[\mathrm{K}\left(y_{i}, y_{j}\right)\right]_{i, j=1}^{m}, \quad Y=\left(y_{1}, \ldots, y_{m}\right)
$$

Here the matrix $\mathrm{K}$ is defined in terms of $L$ by the expression

$$
\mathrm{K}=J+(J+L)^{-1}
$$

the Pfaffian expression for $m$-point correlation functions reflects the fact that the Pfaffian $L$ ensembles is a special class of Pfaffian point processes. Different Pfaffian processes were considered previously by Tracy and Widom [56], Rains [49], Olshanski [48, Soshnikov [51].

3.2. Special matrices $L$. Here we assume that sets $\mathfrak{X}, X$ are ordered. Thus if $X=\left(x_{1}, x_{2}, \ldots\right)$ then $x_{1}<x_{2}<\ldots$. For any $x \in \mathfrak{X}$ we denote by ${ }^{l} x$ the immediate left neighbor of $X$ and by ${ }^{r} x$ the immediate right neighbor of $x$. For example, if $\mathfrak{X}=\mathbb{Z}$ then ${ }^{l} x=x-1$ and ${ }^{r} x=x+1$.

Given a fixed splitting of $\mathfrak{X}$ into positive and negative parts, $\mathfrak{X}=\mathfrak{X}_{-} \sqcup \mathfrak{X}_{+}$, we denote by $\mathfrak{x}$ the minimal (left most) element of $\mathfrak{X}_{+}$. We introduce the parity on the sets $\mathfrak{X}_{ \pm}$, referring to the minimal elements of these sets as odd elements.

According to the decomposition of the set $\mathfrak{X}, \mathfrak{X}=\mathfrak{X}_{-} \sqcup \mathfrak{x} \sqcup \mathfrak{X}_{+} \backslash \mathfrak{x}$, we write the matrix $L$ in the block form:

$$
L=\left[\begin{array}{ccc}
L_{--} & L_{-0} & L_{-+} \\
L_{0-} & L_{00} & L_{0+} \\
L_{+-} & L_{+0} & L_{++}
\end{array}\right]
$$

We are interested in the matrices $L$ defined by

$$
L=\left[\begin{array}{ccc}
\mathrm{E} & \mathrm{A} & \mathrm{B} \\
-\mathrm{A}^{T} & 0 & 0 \\
-\mathrm{B}^{T} & 0 & 0
\end{array}\right]
$$

As usual, here E, A, B are the matrices with $2 \times 2$ block elements. Specifically,

$$
\begin{gathered}
\mathrm{E}(x, y)=\left[\begin{array}{cc}
\epsilon(x, y) & 0 \\
0 & 0
\end{array}\right], x, y \in \mathfrak{X}_{-} \\
\mathrm{A}(x, y)=\left[\begin{array}{cc}
\epsilon(x, y) & 0 \\
0 & \frac{h(x) h(y)}{x-y}
\end{array}\right], x \in \mathfrak{X}_{-}, y=\mathfrak{x} . \\
\mathrm{B}(x, y)=\left[\begin{array}{cc}
0 & 0 \\
\frac{h(x) h(y)}{x-y} & \frac{h(x) h\left({ }^{l} y\right)}{x-{ }^{l} y}
\end{array}\right], x \in \mathfrak{X}_{-}, y \in \mathfrak{X}_{+} \backslash \mathfrak{x} .
\end{gathered}
$$

The two-point function $\epsilon(x, y)$ in equations above is antisymmetric, $\epsilon(x, y)=-\epsilon(y, x)$. When $x<y$,

$$
\epsilon(x, y)= \begin{cases}1, & x \text {-odd, } y-\text { even } \\ 0, & \text { otherwise. }\end{cases}
$$

The function $h$ is nonnegative on $\mathfrak{X}$.

Configurations $X \in \mathfrak{X}$ can be divided into two classes. The first class consists of configuration which do not include the point $\mathfrak{x}$. Such configurations have the form $X=X_{-} \sqcup X_{+}, X_{+}=$ 
$\left(x_{1}^{+}, x_{2}^{+}, \ldots\right), x_{1}^{+}>\mathfrak{x}$. The second class consists of configurations including the point $\mathfrak{x}$. For such configuration $X_{+}=\left(\mathfrak{x}, x_{1}^{+}, x_{2}^{+}, \ldots\right)$. For any $X \subset \mathfrak{X}$ denote by $\tilde{X}$ the configuration defined by

$$
\begin{gathered}
\tilde{X}=\tilde{X}_{-} \sqcup \tilde{X}_{+} \\
\tilde{X}_{-}=X_{-} \\
\tilde{X}_{+}= \begin{cases}\left({ }^{l} x_{1}^{+}, x_{1}^{+},{ }^{l} x_{2}^{+}, x_{2}^{+}, \ldots\right), & X_{+} \cap \mathfrak{x}=\emptyset \\
\left(\mathfrak{x},{ }^{l} x_{1}^{+}, x_{1}^{+},{ }^{l} x_{2}^{+}, x_{2}^{+}, \ldots\right), & X_{+} \cap \mathfrak{x} \neq \emptyset\end{cases}
\end{gathered}
$$

Definition 3.2.1. We say that $X \in \operatorname{Conf}^{L}(\mathfrak{X})$ if

- $X_{+}=\left(x_{1}^{+}<x_{2}^{+}<\ldots\right)$

- all points of $\tilde{X}_{+}$are different

- $\left|\tilde{X}_{-}\right|=\left|\tilde{X}_{+}\right|$

- $\tilde{X}_{-}=\left(x_{1}^{-}<x_{2}^{-}<\ldots\right)$, where $x_{i}^{-}$has the same parity as $i$.

This definition is justified by the following statement.

Theorem 3.2.2. With L given by equations (3.2.1)-(3.2.6) we have

$$
\operatorname{Prob}_{L}(X)=\frac{1}{\operatorname{Pf}(J+L)} \frac{V\left(\tilde{X}_{-}\right) V\left(\tilde{X}_{+}\right)}{\prod\left(\tilde{X}_{+} ; \tilde{X}_{-}\right)} h(\tilde{X})
$$

for $X \in \operatorname{Conf}^{L}(\mathfrak{X})$ and 0 for all other $X \in \mathfrak{X}$.

Proof. The positive integer $d=\left|\tilde{X}_{-}\right|=\left|\tilde{X}_{+}\right|$can be even or odd, depending on whether $X$ includes the point $\mathfrak{x}$ or not. According to that consider two cases.

Case 1. $X \cap \mathfrak{x}=\emptyset$

Given copies $X^{\prime}, X^{\prime \prime}$ of $X \in \mathfrak{X}$ in $\mathfrak{X}^{\prime}, \mathfrak{X}^{\prime \prime}$ we denote by $X^{\prime} \uplus X^{\prime \prime}$ the set $\left(x_{1}^{\prime}, x_{1}^{\prime \prime}, x_{2}^{\prime}, x_{2}^{\prime \prime}, \ldots\right)$. Then we have

$$
\begin{aligned}
\operatorname{Pf} L(X \mid X) & =\operatorname{Pf} L\left[X_{-} \sqcup X_{+} \mid X_{-} \sqcup X_{+}\right] \\
& =\operatorname{Pf} L\left[\left(X_{-}^{\prime} \uplus X_{-}^{\prime \prime}\right) \sqcup\left(X_{+}^{\prime} \uplus X_{+}^{\prime \prime}\right) \mid\left(X_{-}^{\prime} \uplus X_{-}^{\prime \prime}\right) \sqcup\left(X_{+}^{\prime} \uplus X_{+}^{\prime \prime}\right)\right] \\
& =(-)^{\frac{d(d-1)}{2}} \cdot \operatorname{Pf} L\left[X_{-}^{\prime} \mid X_{-}^{\prime}\right] \\
& \times \operatorname{Pf} L\left[X_{-}^{\prime \prime} \sqcup\left(X_{+}^{\prime} \uplus X_{+}^{\prime \prime}\right) \mid X_{-}^{\prime \prime} \sqcup\left(X_{+}^{\prime} \uplus X_{+}^{\prime \prime}\right)\right]
\end{aligned}
$$

as the function $L(x, y)=0$ for any $x \in X_{-}^{\prime}$ and any $y$ which does not belong to $X_{-}^{\prime}$ (see equations (3.1.1) -(3.1.2) and (3.2.2)-(3.2.6) $)$. We note that $L(x, y)=0$, if $x, y \in \mathfrak{X}_{-}^{\prime \prime}$, or if $x, y \in X_{+}^{\prime} \uplus X_{+}^{\prime \prime}$ Therefore $\left|X_{-}^{\prime \prime}\right|=\left|X_{+}^{\prime}\right|+\left|X_{+}^{\prime \prime}\right|$, or $\left|X_{-}\right|=2\left|X_{+}\right|$, which means that $\left|\tilde{X}_{+}\right|=\left|\tilde{X}_{-}\right|$.

Consider Pf $L\left[X_{-}^{\prime} \mid X_{-}^{\prime}\right]$. Note that the matrix $L\left[X_{-}^{\prime} \mid X_{-}^{\prime}\right]$ is even dimensional, if $\left|X_{-}\right|=2\left|X_{+}\right|$. Moreover the matrix $L\left[X_{-}^{\prime} \mid X_{-}^{\prime}\right]$ is the matrix whose $(i, j)$ entry is, by definition, given by $\epsilon\left(x_{i}^{-}, x_{j}^{-}\right)$. Clearly, if $x_{1}^{-}$is even, the first row of this matrix consists of only zeros. Thus, if $\operatorname{Pf} L\left[X_{-}^{\prime} \mid X_{-}^{\prime}\right] \neq 0$, $x_{1}^{-}$must be odd. Now assume that $x_{2 i-1}^{-}$and $x_{2 i}^{-}$have the same parity. In this case $(2 i-1)^{\mathrm{st}}$ and $2 i^{\text {th }}$ rows of the matrix $L\left[X_{-}^{\prime} \mid X_{-}^{\prime}\right]$ are equal to each other. Therefore, if $\operatorname{Pf} L\left[X_{-}^{\prime} \mid X_{-}^{\prime}\right] \neq 0$ the elements of the set $\tilde{X}_{-}=\left(x_{1}^{-}, x_{2}^{-}, \ldots\right)$ are such that $x_{1}^{-}$is odd, $x_{2}^{-}$is even, $x_{3}^{-}$is odd and so on. This proves the condition on the parity for the configurations in $\operatorname{Con} f^{L}(\mathfrak{X})$. Moreover, using the 
definition of Pfaffian it is not hard to conclude that $\operatorname{Pf} L\left[X_{-}^{\prime} \mid X_{-}^{\prime}\right]=1$ for the configurations with non-zero probabilities.

Since $\left|X_{-}^{\prime \prime}\right|=\left|X_{+}^{\prime}\right|+\left|X_{+}^{\prime \prime}\right|$ the matrix $L\left[X_{-}^{\prime \prime} \sqcup\left(X_{+}^{\prime} \uplus X_{+}^{\prime \prime}\right) \mid X_{-}^{\prime \prime} \sqcup\left(X_{+}^{\prime} \uplus X_{+}^{\prime \prime}\right)\right]$ has the block structure:

$$
\left[\begin{array}{cc}
\mathbb{O}_{d \times d} & Q_{d \times d} \\
-Q_{d \times d}^{T} & \mathbb{O}_{d \times d}
\end{array}\right]
$$

with

$$
Q_{d \times d}=\left[\begin{array}{ccccc}
\frac{h\left(x_{1}^{-}\right) h\left(x_{1}^{+}\right)}{x_{1}^{-}-x_{1}^{+}} & \frac{h\left(x_{1}^{-}\right) h\left({ }^{l} x_{1}^{+}\right)}{x_{1}^{-}-{ }^{l} x_{1}^{+}} & \ldots & \frac{h\left(x_{1}^{-}\right) h\left(x_{d / 2}^{+}\right)}{x_{1}^{-}-x_{d / 2}^{+}} & \frac{h\left(x_{1}^{-}\right) h\left({ }^{l} x_{d / 2}^{+}\right)}{x_{1}^{-}-{ }^{l} x_{d / 2}^{+}} \\
\vdots & & & \\
\frac{h\left(x_{d}^{-}\right) h\left(x_{1}^{+}\right)}{x_{d}^{-}-x_{1}^{+}} & \frac{h\left(d_{1}^{-}\right) h\left({ }^{l} x_{1}^{+}\right)}{x_{d}^{-}-{ }^{l} x_{1}^{+}} & \ldots & \frac{h\left(x_{d}^{-}\right) h\left(x_{d / 2}^{+}\right)}{x_{d}^{-}-x_{d / 2}^{+}} & \frac{h\left(x_{d}^{-}\right) h\left({ }^{l} x_{d / 2}^{+}\right)}{x_{d}^{-}-{ }^{l} x_{d / 2}^{+}}
\end{array}\right]
$$

( $d$ is even). Thus we have

$$
\begin{aligned}
\operatorname{Pf} L(X \mid X) & =(-)^{\frac{d(d-1)}{2}} \operatorname{Pf}\left[\begin{array}{cc}
\mathbb{O}_{d \times d} & Q_{d \times d} \\
-Q_{d \times d}^{T} & \mathbb{O}_{d \times d}
\end{array}\right]=\operatorname{det} Q_{d \times d} \\
& =(-)^{\frac{d}{2}}(-)^{\frac{d(d-1)}{2}} \frac{V\left(\tilde{X}_{-}\right) V\left(\tilde{X}_{+}\right)}{\prod\left(\tilde{X}_{+} ; \tilde{X}_{-}\right)} h(\tilde{X})
\end{aligned}
$$

where we have used the formula for the Cauchy determinant, see Appendix. Noting that $(-)^{\frac{d(d-1)}{2}+\frac{d}{2}}=$ $(-)^{\frac{d^{2}}{2}}=1$ (as $d$ is even) we obtain the formula stated in the Theorem.

Case 2. $X \cap \mathfrak{x} \neq 0$

The proof is very similar. We observe that any configuration $X$ has a form

$$
X=X_{-} \sqcup \mathfrak{x} \sqcup X_{+}
$$

Then

$$
\begin{aligned}
& \operatorname{Pf} L(X \mid X)=\operatorname{Pf} L\left[X_{-} \sqcup \mathfrak{x} \sqcup X_{+} \mid X_{-} \sqcup \mathfrak{x} \sqcup X_{+}\right]= \\
& \operatorname{Pf} L\left[\left(X_{-}^{\prime} \uplus X_{-}^{\prime \prime}\right) \sqcup\left(\mathfrak{x}^{\prime}, \mathfrak{x}^{\prime \prime}\right) \sqcup\left(X_{+}^{\prime} \uplus X_{+}^{\prime \prime}\right) \mid\left(X_{-}^{\prime} \uplus X_{-}^{\prime \prime}\right) \sqcup\left(\mathfrak{x}^{\prime}, \mathfrak{x}^{\prime \prime}\right) \sqcup\left(X_{+}^{\prime} \uplus X_{+}^{\prime \prime}\right)\right] \\
& =(-)^{\frac{d(d-1)}{2}} \cdot \operatorname{Pf} L\left[X_{-}^{\prime}, \mathfrak{x}^{\prime} \mid X_{-}^{\prime}, \mathfrak{x}^{\prime}\right] \\
& \times \operatorname{Pf} L\left[X_{-}^{\prime \prime} \sqcup \mathfrak{x}^{\prime \prime} \sqcup\left(X_{+}^{\prime} \uplus X_{+}^{\prime \prime}\right) \mid X_{-}^{\prime \prime} \sqcup \mathfrak{x}^{\prime \prime} \sqcup\left(X_{+}^{\prime} \uplus X_{+}^{\prime \prime}\right)\right]
\end{aligned}
$$

Clearly, $\left|X_{-}^{\prime \prime}\right|=\left|\mathfrak{x}^{\prime} \sqcup\left(X_{+}^{\prime} \uplus X_{+}^{\prime \prime}\right)\right|$, otherwise Pf $L(X \mid X)=0$. Thus $\tilde{X}_{-}$consists of odd number of elements, and $\left|\tilde{X}_{-}\right|=\left|\tilde{X}_{+}\right|=d, d$ is odd, and we repeat the same computations as in the previous case.

3.3. Pfaffian $\hat{L}$-ensembles. Given $\mathfrak{X}=\mathfrak{X}_{-} \sqcup \mathfrak{X}_{+}$consider a different splitting of $\mathfrak{X}, \mathfrak{X}=\hat{\mathfrak{X}}_{-} \sqcup \hat{\mathfrak{X}}_{+}$. Here $\hat{\mathfrak{X}}_{+}=\mathfrak{X}_{+} \sqcup \mathfrak{X}_{0}, \hat{\mathfrak{X}}_{-}=\mathfrak{X}_{-} \backslash \mathfrak{X}_{0}$, and the set $\mathfrak{X}_{0}$ is a subset of $\mathfrak{X}_{-}$. Assume that $\mathfrak{X}_{0}$ consists of $2 S$ rightmost points of $\mathfrak{X}_{-}$.

Define a new matrix $\hat{L}$ on $\mathfrak{X}$ in such a way that it has the same structure with respect to the decomposition $\mathfrak{X}=\hat{\mathfrak{X}}_{-} \sqcup \hat{\mathfrak{x}} \sqcup \hat{\mathfrak{X}}_{+} \backslash \hat{\mathfrak{x}}$ as the matrix $L$ with respect to the decomposition $\mathfrak{X}=$ 
$\mathfrak{X}_{-} \sqcup \mathfrak{x} \sqcup \mathfrak{X}_{+} \backslash \mathfrak{x}$. (Here $\hat{\mathfrak{x}}$ stands for the minimal (left most) element of $\hat{\mathfrak{X}}_{+}$). Then $\hat{L}$ is given by

$$
\hat{L}=\left[\begin{array}{ccc}
\hat{\mathrm{E}} & \hat{\mathrm{A}} & \hat{\mathrm{B}} \\
-\hat{\mathrm{A}}^{T} & 0 & 0 \\
-\hat{\mathrm{B}}^{T} & 0 & 0
\end{array}\right]
$$

Here $\hat{E}, \hat{A}, \hat{B}$ are the matrices with $2 \times 2$ block elements. Specifically,

$$
\begin{gathered}
\hat{\mathrm{E}}(x, y)=\left[\begin{array}{cc}
\epsilon(x, y) & 0 \\
0 & 0
\end{array}\right], x, y \in \hat{\mathfrak{X}}_{-} \\
\hat{\mathrm{A}}(x, y)=\left[\begin{array}{cc}
\epsilon(x, y) & 0 \\
0 & \frac{\hat{h}(x) \hat{h}(y)}{x-y}
\end{array}\right], x \in \hat{\mathfrak{X}}_{-}, y=\hat{\mathfrak{x}}_{.} \\
\hat{\mathrm{B}}(x, y)=\left[\begin{array}{cc}
0 & 0 \\
\frac{\hat{h}(x) \hat{h}(y)}{x-y} & \frac{\hat{h}(x) \hat{h}\left({ }^{l} y\right)}{x-{ }^{l} y}
\end{array}\right], x \in \hat{\mathfrak{X}}_{-}, y \in \hat{\mathfrak{X}}_{+} \backslash \hat{\mathfrak{x}} .
\end{gathered}
$$

If $\hat{h}$ is nonnegative on $\mathfrak{X}$ the matrix $\hat{L}$ defines a Pfaffian ensemble on $\mathfrak{X}$ which we call $\hat{L}$-ensemble.

We introduce the set of point configurations $\operatorname{Conf} f^{\hat{L}}(\mathfrak{X})$ in the same way as the set $\operatorname{Conf} f^{L}(\mathfrak{X})$ for the Pfaffian $L$-ensemble was introduced. Namely, for any $Z \in \mathfrak{X}$ denote by $\tilde{Z}$ the configuration defined by

$$
\begin{gathered}
\tilde{Z}=\tilde{Z}_{-} \sqcup \tilde{Z}_{+} \\
\tilde{Z}_{-}=Z_{-} \\
\tilde{Z}_{+}= \begin{cases}\left({ }^{l} z_{1}^{+}, z_{1}^{+}, l{ }^{l} z_{2}^{+}, z_{2}^{+}, \ldots\right), & Z_{+} \cap \hat{\mathfrak{x}}=\emptyset \\
\left(\hat{\mathfrak{x}}, l, z_{1}^{+}, z_{1}^{+}, l{ }_{2}^{+}, z_{2}^{+}, \ldots\right), & Z_{+} \cap \hat{\mathfrak{x}} \neq \emptyset\end{cases}
\end{gathered}
$$

Here $Z_{ \pm}=Z \cap \hat{\mathfrak{X}}_{ \pm}$.

Definition 3.3.1. We say that $Z \in \operatorname{Conf}^{\hat{L}}(\mathfrak{X})$ if

- all points of $\tilde{Z}_{+}$are different

- $\left|\tilde{Z}_{-}\right|=\left|\tilde{Z}_{+}\right|$

- $\tilde{Z}_{-}=\left(z_{1}^{-}<z_{2}^{-}<\ldots\right)$, where $z_{i}^{-}$has the same parity as $i$.

Theorem 3.2.2 says that with $\hat{L}$ given by equations (3.3.1)-(3.3.4) we obtain

$$
\operatorname{Prob}_{\hat{L}}(Z)=\frac{1}{\operatorname{Pf}(J+\hat{L})} \frac{V\left(\tilde{Z}_{-}\right) V\left(\tilde{Z}_{+}\right)}{\prod\left(\tilde{Z}_{+} ; \tilde{Z}_{-}\right)} \hat{h}(\tilde{Z})
$$

for $Z \in \operatorname{Con}^{\hat{L}}(\mathfrak{X})$ and 0 for all other $Z \subset \mathfrak{X}$. Given $Z \in \operatorname{Con} f^{\hat{L}}(\mathfrak{X})$ we build from $\tilde{Z}$ a configuration $\tilde{X}$ by the particle-hole involution on $\mathfrak{X}_{0}$ (see Section 2.1 for the definition). We note that the corresponding configuration $X$ does not belong to $\operatorname{Con} f^{L}(\mathfrak{X})$, since the configuration $\tilde{X}$ is unbalanced with respect to the splitting $\mathfrak{X}=\mathfrak{X}_{-} \sqcup \mathfrak{X}_{+}$. Moreover,

- $\left|\tilde{X}_{-}\right|-\left|\tilde{X}_{+}\right|=2 S$

- $\tilde{X}_{+}=\left({ }^{l} x_{1}^{+}, x_{1}^{+},{ }^{l} x_{2}^{+}, x_{2}^{+}, \ldots\right), \quad X_{+} \cap \mathfrak{x}=\emptyset$

- $\tilde{X}_{+}=\left(\mathfrak{x},{ }^{l} x_{1}^{+}, x_{1}^{+},{ }^{l} x_{2}^{+}, x_{2}^{+}, \ldots\right), \quad X_{+} \cap \mathfrak{x} \neq \emptyset$

- $\tilde{X}_{-}=\left(x_{1}^{-}, x_{2}^{-}, \ldots\right), x_{i}^{-}$has the same parity as $i$, 
and all points of $\tilde{X}_{ \pm}$are different.

Define the weight $\hat{h}$ of the $\hat{L}$-ensemble in terms of the weight $h$ of the $L$-ensemble by the formula:

$$
\hat{h}(z)= \begin{cases}h(z) \prod_{y \in \mathfrak{X}_{0}}|z-y|, & z \in \mathfrak{X}_{-} \backslash \mathfrak{X}_{0}, \\ \frac{1}{h(z) \prod_{y \in \mathfrak{X}_{0}, y \neq z}|z-y|}, & z \in \mathfrak{X}_{0}, \\ \frac{h(z)}{\prod_{y \in \mathfrak{X}_{0}}|z-y|}, & z \in \mathfrak{X}_{+} .\end{cases}
$$

Note that this formula is identical to (2.3.3) except for the absolute values. We did not need absolute values in (2.3.3) because there the formulas only contain $h^{2}$ and not $h$.

Proposition 3.3.2. With $\hat{h}(z)$ defined by equation 3.3.6) and $\tilde{X}$ constructed from $\tilde{Z}$ by the particle-hole involution on $\mathfrak{X}_{0}$,

$$
\operatorname{Prob}_{\hat{L}}(Z)=\frac{1}{\operatorname{Pf}(J+\hat{L})} \frac{h(\tilde{X})}{\left|V\left(\mathfrak{X}_{0}\right)\right| h\left(\mathfrak{X}_{0}\right)} \frac{\left|V\left(\tilde{X}_{-}\right)\right|\left|V\left(\tilde{X}_{+}\right)\right|}{\prod\left(\tilde{X}_{+}, \tilde{X}_{-}\right)}, Z \in \operatorname{Conf}^{\hat{L}}(\mathfrak{X})
$$

Proof. The probability of the configuration $Z$ can be rewritten in terms of $X$ by the same method as it was done for the case of determinantal ensembles, see Proposition [2.3.1]

3.4. Correlation functions of Pfaffian $L$-ensembles and averages of characteristic polynomials. The goal of this Section is to express the kernel $\mathrm{K}$ of the $m$-point correlation function $\varrho_{m}(Y)$ in terms of averages of characteristic polynomials associated with Pfaffian $L$-ensembles. Namely we want to prove the Pfaffian analog of Proposition 2.4.2

Introduce nonintersecting sets $\alpha^{ \pm}$of complex numbers with nonequal elements,

$$
\alpha_{+}=\left(\alpha_{1}^{+}, \ldots, \alpha_{k}^{+}\right), \alpha_{-}=\left(\alpha_{1}^{-}, \ldots, \alpha_{m}^{-}\right) .
$$

Assume that $\alpha^{ \pm} \cap \mathfrak{X}=0$, and

$$
k-m=\left|\alpha_{+}\right|-\left|\alpha_{-}\right|=2 S, \quad S \in \mathbb{Z} .
$$

Similarly to the case of the determinantal $L$-ensembles we extend the definition of the matrices $\mathrm{K}, L$ as follows. Let $\alpha^{\prime}, \alpha^{\prime \prime}$ denote two copies of the set $\alpha=\alpha_{-} \sqcup \alpha_{+}$. We add to $L$ rows and columns parameterized by $\alpha^{\prime}, \alpha^{\prime \prime}$, and then define new matrix elements of $L$ in accordance with equations (3.2.2)-(3.2.5), where we assume that $\alpha_{-}$is added to $\mathfrak{X}_{-}$, and $\alpha_{+}$is added to $\mathfrak{X}_{+}$. Then we consider the matrix

$$
\mathrm{K}(\alpha \sqcup \mathfrak{X} \mid \alpha \sqcup \mathfrak{X})=J+(J+L(\alpha \sqcup \mathfrak{X} \mid \alpha \sqcup \mathfrak{X}))^{-1} .
$$

Proposition 3.4.1. The Pfaffian of the symmetric $(k+m) \times(k+m)$ submatrix $K\left(\alpha^{\prime} \mid \alpha^{\prime}\right)$ of the matrix $K\left(\alpha^{\prime} \uplus \alpha^{\prime \prime} \sqcup \mathfrak{X}^{\prime} \uplus \mathfrak{X}^{\prime \prime} \mid \alpha^{\prime} \uplus \alpha^{\prime \prime} \sqcup \mathfrak{X}^{\prime} \uplus \mathfrak{X}^{\prime \prime}\right)$ can be given as a normalized average of the functions $E(\cdot, \cdot)$ defined by equations (2.4.2) with respect to the Pfaffian $\hat{L}$-ensemble. Namely,

$$
\begin{aligned}
\operatorname{Pf}\left[K\left(\alpha^{\prime} \mid \alpha^{\prime}\right)\right] & =h(\alpha) \frac{V\left(\alpha_{-}\right) V\left(\alpha_{+}\right)}{\prod\left(\alpha_{-} ; \alpha_{+}\right)}\left[\frac{\operatorname{Pf}(J+\hat{L})}{\operatorname{Pf}(J+L)} h\left(\mathfrak{X}_{0}\right)\left|V\left(\mathfrak{X}_{0}\right)\right|\right] \\
& \times\left[\frac{\prod\left(\alpha_{+} ; \mathfrak{X}_{0}\right)}{\prod\left(\alpha^{-} ; \mathfrak{X}_{0}\right)}\right]\left\langle\frac{E\left(\alpha^{+}, Z\right)}{E\left(\alpha^{-}, Z\right)}\right\rangle_{\hat{L}}
\end{aligned}
$$

Proof. Combining equations (6.8.3) and (6.9.1) of Appendix we obtain the following formula:

$$
\operatorname{Pf} K\left[\alpha^{\prime} \mid \alpha^{\prime}\right]=\frac{1}{\operatorname{Pf}(J+L)} \sum_{X \in \mathfrak{X}} \operatorname{Pf} L\left[\alpha^{\prime \prime} \sqcup X^{\prime} \uplus X^{\prime \prime} \mid \alpha^{\prime \prime} \sqcup X^{\prime} \uplus X^{\prime \prime}\right]
$$


Here $\alpha$ is a set with even $|\alpha|=k+m$, and $\alpha^{\prime}, \alpha^{\prime \prime}$ are two copies of $\alpha$. Now we compute the Pfaffian in the sum using similar arguments as in the proof of Theorem 3.2 .2 .

Assume first that $X \cap \mathfrak{x}=0$. We denote $\mathrm{E}(\beta) \equiv L(\beta \mid \beta)$ for any set $\beta$. Then the Pfaffian in the sum is

$$
\begin{aligned}
& \operatorname{Pf} \mathrm{E}\left[\alpha^{\prime \prime} \sqcup X^{\prime} \uplus X^{\prime \prime}\right] \\
& =\operatorname{Pf} \amalg\left[\alpha_{-}^{\prime \prime} \sqcup \alpha_{+}^{\prime \prime} \sqcup X_{-}^{\prime} \uplus X_{-}^{\prime \prime} \sqcup X_{+}^{\prime} \uplus X_{+}^{\prime \prime}\right] \\
& =\operatorname{Pf} €\left[\alpha_{-}^{\prime \prime} \sqcup X_{-}^{\prime} \uplus X_{-}^{\prime \prime} \sqcup X_{+}^{\prime} \uplus X_{+}^{\prime \prime} \sqcup \alpha_{+}^{\prime \prime}\right] \\
& =(-)^{\frac{\left|X_{-}\right|\left(\left|X_{-}\right|-1\right)}{2}}(-)^{\left|\alpha_{-}\right|\left|X_{-}\right|} \operatorname{Pf} €\left[X_{-}^{\prime} \sqcup \alpha_{-}^{\prime \prime} \sqcup X_{-}^{\prime \prime} \sqcup X_{+}^{\prime} \uplus X_{+}^{\prime \prime} \sqcup \alpha_{+}^{\prime \prime}\right] \\
& =(-)^{\frac{\left|X_{-}\right|\left(\left|X_{-}\right|-1\right)}{2}}(-)^{\left|\alpha_{-}\right|\left|X_{-}\right|} \operatorname{Pf} \mathrm{E}\left[X_{-}^{\prime}\right] \operatorname{Pf} €\left[\alpha_{-}^{\prime \prime} \sqcup X_{-}^{\prime \prime} \sqcup X_{+}^{\prime} \uplus X_{+}^{\prime \prime} \sqcup \alpha_{+}^{\prime \prime}\right]
\end{aligned}
$$

By equations (3.2.2)-(3.2.5) $L\left(*_{-}, *_{+}\right)=0$. Thus, the last expression does not equal to zero only if $\left|\alpha_{-}\right|+\left|X_{-}\right|=\left|\alpha_{+}\right|+2\left|X_{+}\right|$, and $X_{-}=\left(x_{1}^{-}, x_{2}^{-}, \ldots\right)$ is such that $x_{i}^{-}$has the same parity as $i$. Since $\left|\alpha_{+}\right|-\left|\alpha_{-}\right|=2 S, X_{-}$consists of even number of elements. We note that $\operatorname{Pf} L\left(X_{-}^{\prime}\right)=1$. Then

$$
\begin{aligned}
\operatorname{Pf} €\left[\alpha^{\prime \prime} \sqcup X^{\prime} \uplus X^{\prime \prime}\right] & =(-)^{\frac{\left|X_{-}\right|\left(\left|X_{-}\right|-1\right)}{2}}(-)^{\frac{\left|\tilde{X}_{+}\right|}{2}} \\
& \times \frac{V\left(\alpha_{-} \sqcup \tilde{X}_{-}\right) V\left(\tilde{X}_{+} \sqcup \alpha_{+}\right)}{\prod\left(\alpha_{-} \sqcup \tilde{X}_{-} ; \tilde{X}_{+} \sqcup \alpha_{+}\right)} \\
& \times h(\tilde{X}) h(\alpha) \\
& =\left(-\frac{\left|X_{-}\right|\left(\left|X_{-}\right|-1\right)}{2}(-)^{\frac{\left|\tilde{X}_{+}\right|}{2}}\right. \\
& \times\left[h(\alpha) \frac{V\left(\alpha_{-}\right) V\left(\alpha_{+}\right)}{\prod\left(\alpha_{-} ; \alpha_{+}\right)}\right] \\
& \times\left[\frac{E\left(\alpha_{+}, \tilde{X}\right)}{E\left(\alpha_{-}, \tilde{X}\right)}\right] \frac{V\left(\tilde{X}_{-}\right) V\left(\tilde{X}_{+}\right)}{\prod\left(\tilde{X}_{+} ; \tilde{X}_{-}\right)} h(\tilde{X})
\end{aligned}
$$

It remains to see that the sign cancels out. We know that $\left|\tilde{X}_{-}\right|-\left|\tilde{X}_{+}\right|=\left|\alpha_{+}\right|-\left|\alpha_{-}\right|=2 S$ and $\left|\tilde{X}_{-}\right|$is even. Then

$$
\begin{aligned}
& (-)^{\frac{\left|X_{-}\right|\left(\left|X_{-}\right|-1\right)}{2}}(-)^{\frac{\left|\tilde{X}_{+}\right|}{2}}=(-)^{S} \\
& \frac{V\left(\tilde{X}_{-}\right) V\left(\tilde{X}_{+}\right)}{\prod\left(\tilde{X}_{+} ; \tilde{X}_{-}\right)}=(-)^{S} \frac{\left|V\left(\tilde{X}_{-}\right)\right|\left|V\left(\tilde{X}_{+}\right)\right|}{\prod\left(\tilde{X}_{+} ; \tilde{X}_{-}\right)}
\end{aligned}
$$

and the result is

$$
\begin{aligned}
\operatorname{Pf} \mathrm{L}\left[\alpha^{\prime \prime} \sqcup X^{\prime} \uplus X^{\prime \prime}\right] & =h(\alpha) \frac{V\left(\alpha_{-}\right) V\left(\alpha_{+}\right)}{\prod\left(\alpha_{-} ; \alpha_{+}\right)} \\
\times & {\left[\frac{E\left(\alpha_{+}, \tilde{X}\right)}{E\left(\alpha_{-}, \tilde{X}\right)}\right] \frac{\left|V\left(\tilde{X}_{-}\right)\right|\left|V\left(\tilde{X}_{+}\right)\right|}{\prod\left(\tilde{X}_{+} ; \tilde{X}_{-}\right)} h(\tilde{X}) . }
\end{aligned}
$$

Now we apply Proposition 3.3 .2 and obtain formula (3.4.4). The case $X \cap \mathfrak{x} \neq 0$ is considered in the same way. 
3.5. Discrete symplectic ensemble. Given $\mathfrak{X}$ denote by $\operatorname{Con} f_{2 N}^{(4)}(\mathfrak{X})$ the following set of point configurations:

$$
\operatorname{Conf}_{2 N}^{(4)}(\mathfrak{X})=\left\{X \subset \mathfrak{X} \mid X=\left({ }^{l} x_{1}<x_{1}<\ldots<{ }^{l} x_{N}<x_{N}\right)\right\}
$$

Assume that a nonnegative function $f$ is given on $\mathfrak{X}$, which does not vanish at least at $2 N$ distinct points.

Definition 3.5.1. The point process which lives on $\operatorname{Con} f_{2 N}^{(4)}(\mathfrak{X})$ and for which the probability of a configuration $X$ is given by

$$
\operatorname{Prob}(X)=\left[c_{N}^{(4)}\right]^{-1} \prod_{i=1}^{N} f\left(x_{i}\right)|V(X)|, \quad X \in \operatorname{Conf}_{2 N}^{(4)}(\mathfrak{X})
$$

will be called $2 N$ point discrete symplectic ensemble and will be denoted by $\triangle_{2 N}^{(4)}(f)$. Here $c_{N}^{(4)}$ is a normalization constant.

Remark 3.5.2. Rewrite $|V(X)|$ as follows

$$
|V(X)|=\left|\prod_{i<j}\left(x_{i}-x_{j}\right)\left({ }^{l} x_{i}-{ }^{l} x_{j}\right)\left({ }^{l} x_{i}-x_{j}\right)\left(x_{i}-{ }^{l} x_{j}\right) \prod_{i=1}^{N}\left({ }^{l} x_{i}-x_{i}\right)\right| .
$$

In the continuous limit the points ${ }^{l} x$ and $x$ get closer and closer to each other, and all differences ${ }^{l} x_{i}-x_{i}$ turns into the same small constant, say $\epsilon$. Thus the degree of the Vandermonde determinant turns into four, and the probability distribution (3.5.1) takes the same form as the probability distribution of eigenvalues for the symplectic ensemble of the Random Matrix Theory (see, for example, Mehta [4], Chapter 3).

For a symmetric function $g(X)=g\left({ }^{l} x_{1}, x_{1}, \ldots,{ }^{l} x_{N}, x_{N}\right)$ of points of the configuration $X \in$ Con $f_{2 N}^{(4)}(\mathfrak{X})$, the average with respect to the discrete symplectic ensemble is defined by

$$
\langle g\rangle_{\triangle_{2 N}^{(4)}(f)}=\left[c_{N}^{(4)}\right]^{-1} \sum_{X \in C o n f_{2 N}^{(4)}(\mathfrak{X})} g(X)|V(X)| f(X)
$$

If $\mathfrak{X}$ is a lattice then in the continuous limit we introduce the skew symmetric inner product for arbitrary functions $g_{1}, g_{2}$ on $\mathfrak{X}$

$$
\left\langle g_{1}, g_{2}\right\rangle=\sum_{x \in \dot{\mathfrak{X}}}\left(g_{1}\left({ }^{l} x\right) g_{2}(x)-g_{2}\left({ }^{l} x\right) g_{1}(x)\right) f\left({ }^{l} x\right) f(x)
$$

where $\dot{\mathfrak{X}}$ denotes $\mathfrak{X}$ without the leftmost point. If

$$
\operatorname{det}\left\|\left\langle x^{i}, x^{j}\right\rangle\right\|_{i, j=1}^{2 n} \neq 0
$$

then a family of monic skew orthogonal polynomials associated with the discrete symplectic ensemble can be constructed.

Definition 3.5.3. For $i=0,1,2, \ldots$ let $p_{2 i}, p_{2 i+1}$ be monic polynomials of the degrees $2 i$ and $2 i+1$, which satisfy the conditions

- $\left\langle p_{2 i}, p_{2 j}\right\rangle=0,\left\langle p_{2 i+1}, p_{2 j+1}\right\rangle=0$

- $\left\langle p_{2 i}, p_{2 j+1}\right\rangle=h_{i} \delta_{i j}$.

The family $\left\{p_{2 i}, p_{2 i+1}\right\}$ will be called the family of the skew orthogonal polynomials.

Remark 3.5.4. The skew orthogonal polynomials are defined up to the replacement

$$
p_{2 j+1} \rightarrow p_{2 j+1}+\text { const } p_{2 j} .
$$


Lemma 3.5.5. For arbitrary functions $\phi_{i}(x), i=1, \ldots, 2 N$ and an antisymmetric two point function $\epsilon(x, y)$ the following identity is valid

$$
\sum_{x_{1}, \ldots, x_{2 N} \in \mathfrak{X}} \operatorname{Pf}\left[\epsilon\left(x_{i}, x_{j}\right)\right]_{i, j=1}^{2 N} \operatorname{det}\left[\phi_{i}\left(x_{j}\right)\right]_{i, j=1}^{2 N}=(2 N) ! \operatorname{Pf}\left[\left\langle\phi_{i}, \phi_{j}\right\rangle\right]_{i, j=1}^{2 N}
$$

where

$$
\left\langle\phi_{i}, \phi_{j}\right\rangle=\sum_{x, y \in \mathfrak{X}} \epsilon(x, y) \phi_{i}(x) \phi_{j}(y)
$$

This is a well known de Bruijn identity, see e.g. de Bruijn [19], Tracy and Widom [56], Baik and Rains [7, Rains [4]. This statement readily implies, (see Rains [49], Corollary 1.3):

Corollary 3.5.6. Let $\phi_{1}, \ldots, \phi_{2 N} ; \psi_{1}, \ldots, \psi_{2 N}$ be arbitrary functions on $\mathfrak{X}$. We set

$$
\underline{\phi}(.)=\left[\begin{array}{c}
\phi_{1}(.) \\
\vdots \\
\phi_{2 N}(.)
\end{array}\right], \quad \underline{\psi}(.)=\left[\begin{array}{c}
\psi_{1}(.) \\
\vdots \\
\psi_{2 N}(.)
\end{array}\right]
$$

and introduce the matrix $A=\left[A_{i j}\right]_{i, j=1}^{2 N}$ :

$$
A_{i j}=\sum_{x \in \mathfrak{X}} \phi_{i}(x) \psi_{j}(x)-\phi_{j}(x) \psi_{i}(x)
$$

Then

$$
\sum_{\substack{x_{1}<\ldots<x_{N} \\ x_{i} \in \mathfrak{X}, i=\overline{1, N}}} \operatorname{det}\left[\underline{\phi}\left(x_{1}\right), \underline{\psi}\left(x_{1}\right), \ldots, \underline{\phi}\left(x_{N}\right), \underline{\psi}\left(x_{N}\right)\right]=\operatorname{Pf} A
$$

The following statement is well known as well, and we give a proof for the reader's convenience.

Proposition 3.5.7. The normalization constant $c_{N}^{(4)}$ is equal to the product of $\left\langle p_{2 i}, p_{2 i+1}\right\rangle$, i.e.

$$
c_{N}^{(4)}=\prod_{i=0}^{N-1} h_{i}, \quad h_{i}=\left\langle p_{2 i}, p_{2 i+1}\right\rangle
$$

Proof. Set

$$
\pi_{i-1}(x)=x^{i-1}+\ldots, i=1, \ldots, 2 N .
$$

This gives a system of polynomials of degrees $0, \ldots, 2 N-1$ with the highest coefficients equal to one. The constant $c_{N}^{(4)}$ is the average over point configurations which includes the absolute value of the Vandermonde determinant and the product of weights. Rewrite the absolute value of the Vandermonde determinant in terms of the polynomials $\pi_{j}$. In particular these polynomials can be chosen to be the skew symmetric orthogonal polynomials with respect to the weight $f$ in the average. Then we obtain

$$
c_{N}^{(4)}=\sum_{\substack{x_{1}<\ldots<x_{N} \\ x_{i} \in \dot{\mathfrak{X}}, i=\overline{1, N}}} \operatorname{det}\left[\underline{\phi}\left(x_{1}\right), \underline{\psi}\left(x_{1}\right), \ldots, \underline{\phi}\left(x_{N}\right), \underline{\psi}\left(x_{N}\right)\right]
$$

where we have introduced

$$
\phi_{i}(x)=p_{i-1}\left({ }^{l} x\right) f\left({ }^{l} x\right), \quad \psi_{i}(x)=p_{i-1}(x) f(x), \quad i=\overline{1,2 N}
$$

Now we apply Corollary 3.5 .6 and prove the Proposition.

Given $X \in \operatorname{Con}_{2 N}^{(4)}(\mathfrak{X})$ and a complex parameter $\zeta$ we define the characteristic polynomial

$$
d(\zeta)=\prod(\zeta ; X)
$$

The following analog of Heine's identity is also well known, see e. g. Eynard [26], Forrester [29]. 
Proposition 3.5.8.

$$
p_{2 N}(\zeta)=\langle d(\zeta)\rangle_{\triangle_{2 N}^{(4)}(f)}
$$

Proof. The average is a sum over the variables $x_{1}, \ldots, x_{N}$ which take values in $\dot{\mathfrak{X}}$. Set

$$
\phi_{i}(x)=p_{i-1}\left({ }^{l} x\right) f\left({ }^{l} x\right), \quad \psi_{i}(x)=p_{i-1}(x) f(x), \quad i=\overline{1,2 N+1}
$$

Then the expression under the sum is equal to the determinant of size $2 N+1 \times 2 N+1$, whose odd columns are $\phi\left(x_{i}\right), i=\overline{1, N}$, the even columns are $\underline{\psi}\left(x_{i}\right), i=\overline{1, N}$, and the last $2 N+1^{\text {th }}$ column is $p_{i-1}(\zeta)$. We represent this determinant as a sum over permutations $\sigma \in S_{2 N+1}$. Note that the sum over all permutations which do not satisfy the following two conditions

- $\sigma(2 N+1)=2 N+1$

- $\{\{\sigma(1), \sigma(2)\}, \ldots,\{\sigma(2 N-1), \sigma(2 N)\}\}=\{\{1,2\}, \ldots,\{2 N-1,2 N\}\}$

vanishes after averaging. This is so because of the orthogonality relations for the skew symmetric orthogonal polynomials. On the other hand, there are $N$ ! permutations which do satisfy the conditions above. All of them give the same contribution, which is proportional to $p_{2 N}(\zeta)$. Obviously, $\langle d(\zeta)\rangle$ is a monic polynomial. This completes the proof.

Proposition 3.5.9. For $\zeta \notin \mathfrak{X}$ the average of $1 / d(\zeta)$ is given by the skew symmetric inner product of $R_{\zeta}(x)=(\zeta-x)^{-1}$ and $p_{2 N-2}(x)$,

$$
\langle 1 / d(\zeta)\rangle_{\triangle_{2 N}^{(4)}(f)}=h_{N-1}^{-1}\left\langle p_{2 N-2}, R_{\zeta}\right\rangle
$$

Proof. The average over the discrete symplectic ensemble is the normalized sum over configurations from the set $\operatorname{Con} f_{2 N}^{(4)}(\mathfrak{X})$. The normalization constant is $c_{N}^{(4)}$. This normalized sum is that over the ordered variables $x_{i} \in \dot{\mathfrak{X}}, i=\overline{1, N}$. We have a symmetric function of variables $x_{i}$ under the sum, so we can remove the ordering of the variables changing the normalization constant from $c_{N}^{(4)}$ to $N ! c_{N}^{(4)}$

The key observation which helps to compute the average is the following one. The expression under the sum contains the absolute value of the Vandermonde determinant, the product of weights and $1 / d(\zeta)$. This expression can be simplified if we expand $1 / d(\zeta)$ into partial fractions,

$$
\begin{aligned}
d^{-1}(\zeta) & =\sum_{\nu=1}^{N} \frac{1}{\zeta-{ }^{l} x_{\nu}} \frac{1}{\prod_{j=1}^{N}\left({ }^{l} x_{\nu}-x_{j}\right) \prod_{\substack{j=1 \\
j \neq \nu}}^{N}\left({ }^{l} x_{\nu}-{ }^{l} x_{j}\right)} \\
& +\sum_{\nu=1}^{N} \frac{1}{\zeta-x_{\nu}} \frac{1}{\prod_{j=1}^{N}\left(x_{\nu}-x_{j}\right) \prod_{\substack{j=1 \\
j \neq \nu}}^{N}\left(x_{\nu}-{ }^{l} x_{j}\right)}
\end{aligned}
$$

We note that each term of the first sum and each term of the second sum gives the same contribution to the average. Indeed, we always can make the change of variables under which $x_{\nu}$ becomes $x_{1}$, and ${ }^{l} x_{\nu}$ becomes ${ }^{l} x_{1}$, thanks to the symmetry of the involved functions under permutations of variables. Therefore the average will consist of two terms. The first term is

$$
\left[c_{N}^{(4)} N !\right]^{-1} \sum_{x_{1}, \ldots, x_{N}}-N R_{\zeta}\left({ }^{l} x_{1}\right) \hat{d}\left(x_{1}\right)\left|V\left({ }^{l} x_{2}, x_{2}, \ldots,{ }^{l} x_{N}, x_{N}\right)\right| \cdot f(X)
$$

where we have denoted $\hat{d}\left(x_{1}\right)=\prod_{j=2}^{N}\left(x_{1}-x_{j}\right)\left(x_{1}-{ }^{l} x_{j}\right)$. The second term is

$$
\left[c_{N}^{(4)} N !\right]^{-1} \sum_{x_{1}, \ldots, x_{N}} N R_{\zeta}\left(x_{1}\right) \hat{d}\left({ }^{l} x_{1}\right)\left|V\left({ }^{l} x_{2}, x_{2}, \ldots,{ }^{l} x_{N}, x_{N}\right)\right| \cdot f(X)
$$


The computation of the sums is reduced to the computation of averages of characteristic polynomials over configurations $X \in \operatorname{Conf}_{2 N-2}^{(4)}(\mathfrak{X})$. The previous Proposition says that these averages are skew symplectic polynomials of the degree $2 N-2$. The remaining sum over $x_{1}$ gives us the skew symmetric product in the righthand side of formula (3.5.16).

Given the family of the skew symmetric orthogonal polynomials with respect to the weight $f$ introduce the Christoffel-Darboux type kernel

$$
K_{N}^{C D, 4}(\zeta, \eta)=\sum_{i=0}^{N-1} \frac{p_{2 i+1}(\zeta) p_{2 i}(\eta)-p_{2 i+1}(\eta) p_{2 i}(\zeta)}{h_{i}}
$$

Proposition 3.5.10. For $\zeta, \eta \notin \mathfrak{X}$, the averages of products and ratios of two characteristic polynomials with respect to the discrete symplectic ensembles are given in terms of $K_{N}^{C D, 4}$ and its pairings with $R_{\zeta}=(\zeta-x)^{-1}, R_{\eta}=(\eta-x)^{-1}$ :

$$
\begin{gathered}
\langle d(\zeta) d(\eta)\rangle_{\triangle_{2 N}^{(4)}(f)}=\frac{h_{N}}{\zeta-\eta} K_{N+1}^{C D, 4}(\zeta, \eta) \\
\left\langle\frac{d(\eta)}{d(\zeta)}\right\rangle_{\triangle_{2 N}^{(4)}(f)}=h_{N-1}^{-1}\left\langle\frac{1}{R_{\eta}(.)}\langle d(\eta) d(.)\rangle_{\triangle_{2 N-2}^{(4)}(f)}, \frac{R_{\zeta}(.)}{R_{\eta}(.)}\right\rangle \\
\left\langle\frac{1}{d(\eta) d(\zeta)}\right\rangle_{\triangle_{2 N}^{(4)}(f)}=h_{N-1}^{-1}\left\langle R_{\eta}(.)\left\langle\frac{d(.)}{d(\eta)}\right\rangle_{\triangle_{2 N-2}^{(4)}(f)}, R_{\eta}(.) R_{\zeta}(.)\right\rangle
\end{gathered}
$$

Remark 3.5.11. Clearly, the first equation remains valid if $\zeta, \eta \in \mathfrak{X}$, and the second equation remains valid for $\eta \in \mathfrak{X}$.

Proof. Basically we proceed as in the computations of averages of the characteristic polynomial and its inverse. Let us first prove the formula for the average of product of characteristic polynomials. This average can be represented as a sum over the variables $x_{i} \in \dot{\mathfrak{X}}, i \in \overline{1, N}$, divided by the normalization constant $c_{N}^{(4)} N$ !. (The ordering of $x_{1}, x_{2}, \ldots, x_{N}$ is removed by $N$ !). Set

$$
\phi_{i}(x)=p_{i-1}\left({ }^{l} x\right) f\left({ }^{l} x\right), \quad \psi_{i}(x)=p_{i-1}(x) f(x), \quad i=\overline{1,2 N+2}
$$

The expression under the sum can be rewritten as the determinant of the $2 N+2 \times 2 N+2$ matrix whose first column is $p_{i}(\zeta)$, the second column is $p_{i}(\eta)$, all other odd columns are $\phi\left(x_{2 i-1}\right)$, and all other even columns are $\underline{\psi}\left(x_{2 i}\right)$. The average now is equal to this sum multiplied by the factor $\left[N ! c_{N}^{(4)}(\zeta-\eta)\right]^{-1}$

Now we rewrite the determinant as a sum over permutations $\sigma \in S_{2 N+2}$, and change the order of sums. The skew symmetric product of $\phi_{2 i-1}$ and $\psi_{2 i}$ gives $h_{i-1}$, and all other skew symmetric products constructed with these functions are zero. Then the sum over all permutations $\sigma$ which do not satisfy the relation

$$
\begin{aligned}
\{\{\sigma(3), \sigma(4)\}, \ldots, & \{\sigma(2 N+1), \sigma(2 N+2)\}\}= \\
& \{\{1,2\}, \ldots,\{2 \widehat{i-1,2} i\}, \ldots,\{2 N+1,2 N\}\}
\end{aligned}
$$

for some $1 \leq i \leq N$ vanishes after the averaging. Consider the permutations which do satisfy this condition. All these permutations transfer the pair $(1,2)$ to the pair $(2 i-1,2 i)$. The sum over such permutations converts the sum over $x_{1}, \ldots, x_{N}$ into the factor $N ! c_{N+1} / h_{i}$, thanks to the orthogonality relations between $\phi$ and $\psi$. The index $i$ takes values from 1 to $N+1$, and we obtain equation (3.5.19).

The second and the third equations are obtained from the first one by the same procedure as that used in the computation of $\langle 1 / d(\zeta)\rangle_{\triangle_{2 N}^{(4)}(f)}$, Proposition 3.5 .9 
Definition 3.5.12. The Cauchy type transform $h_{k}(\zeta)$ of the monic skew orthogonal polynomial $p_{k}$ is the skew symmetric inner product of $R_{\zeta}$ and $p_{k}$,

$$
h_{k}(\zeta)=\left\langle p_{k}, R_{\zeta}\right\rangle
$$

Here $\zeta \notin \mathfrak{X}$, and the skew symmetric product is defined by equation (3.5.4).

Proposition 3.5.13. The averages of two characteristic polynomials with respect to the discrete symplectic ensemble are Christoffel-Darboux type kernels constructed from the skew orthogonal polynomials associated with this ensemble, and their Cauchy type transforms:

- $\langle d(\zeta) d(\eta)\rangle_{\triangle_{2 N}^{(4)}(f)}=\frac{h_{N}}{\zeta-\eta} \sum_{i=0}^{N} \frac{p_{2 i+1}(\zeta) p_{2 i}(\eta)-p_{2 i}(\zeta) p_{2 i+1}(\eta)}{h_{i}}$
- $\left\langle\frac{d(\eta)}{d(\zeta)}\right\rangle_{\triangle_{2 N}^{(4)}(f)}=(\eta-\zeta) \sum_{i=0}^{N-1} \frac{p_{2 i+1}(\eta) h_{2 i}(\zeta)-p_{2 i}(\eta) h_{2 i+1}(\zeta)}{h_{i}}+1$
- $\left\langle\frac{1}{d(\eta) d(\zeta)}\right\rangle_{\triangle_{2 N}^{(4)}(f)}=\frac{1}{h_{N-1}} \frac{1}{\eta-\zeta}\left[\sum_{i=0}^{N-2} \frac{h_{2 i+1}(\eta) h_{2 i}(\zeta)-h_{2 i}(\eta) h_{2 i+1}(\zeta)}{h_{i}}+\left\langle R_{\eta}, R_{\zeta}\right\rangle\right]$

Proof. Straightforward computation of the skew symmetric products in the righthand sides of equations (3.5.19)-(3.5.21).

3.6. Discrete orthogonal ensemble. Any point configuration from the $\operatorname{set} \operatorname{Conf}_{2 N}^{(4)}(\mathfrak{X})$ defines a configuration of holes on $\mathfrak{X}$. All such configurations of holes naturally form a new set of point configurations. We will denote this set by $\operatorname{Con} f_{2 N}^{(1)}(\mathfrak{X})$. Thus,

$$
\operatorname{Conf}_{2 N}^{(1)}(\mathfrak{X})=\left\{Y \subset \mathfrak{X} \mid Y=\mathfrak{X} \backslash X, X \in \operatorname{Conf}_{2 N}^{(4)}(\mathfrak{X})\right\} .
$$

Point configurations from $\operatorname{Con} f_{2 N}^{(1)}$ admit an independent description. Namely, recall that $\mathfrak{X}$ is the ordered set, in which the smallest point is the leftmost one. If we say that the leftmost point of the set $\mathfrak{X}$ is odd, any configuration from the set $\operatorname{Conf}_{2 N}^{(1)}(\mathfrak{X})$ is such that its smallest point is odd, and any two neighboring points are always have different parity. Assume once again that a nonnegative function $f$ is given on $\mathfrak{X}$, which does not vanish at least at $2 N$ points.

Definition 3.6.1. The point process which lives on $\operatorname{Con} f_{2 N}^{(1)}(\mathfrak{X})$, and for which the probability of a configuration $Y$ is given by

$$
\operatorname{Prob}(Y)=\text { const } f(Y) \cdot|V(Y)|, \quad Y \in \operatorname{Conf}_{2 N}^{(1)}(\mathfrak{X})
$$

will be called $2 N$ point discrete orthogonal ensemble and will be denoted by $\triangle_{2 N}^{(1)}(f)$.

Define a two point antisymmetric function $\epsilon(x, y)$ on $\mathfrak{X}$ such that it can be equal only to one, zero, or minus one: for $x<y$

$$
\epsilon(x, y)= \begin{cases}1, & x \text { is odd } \\ 0, & \text { otherwise }\end{cases}
$$

Then the definition of $\triangle_{2 N}^{(1)}(f)$ can be rewritten as

$$
\operatorname{Prob}(Y)=\left[c_{N}^{(1)}\right]^{-1} f(Y) \cdot|V(Y)| \operatorname{Pf}\left[\epsilon\left(y_{i}, y_{j}\right)\right]_{i, j=1}^{N}
$$

where $Y$ is any configuration on $\mathfrak{X}$ which consists of $2 N$ points. This is the consequence of the fact that the Pfaffian in equation (3.6.3) is zero for any $Y \notin C o n f_{2 N}^{(1)}(\mathfrak{X})$, and it is one for any $Y \in \operatorname{Conf}_{2 N}^{(1)}(\mathfrak{X})$.

Remark 3.6.2. In the continuous limit the discrete orthogonal ensemble turns into the orthogonal ensemble of the Random Matrix Theory (see Mehta 44, Chapter 3, for the definition). This is so because the parity condition for the configurations with nonzero probabilities becomes irrelevant in the continuous limit. 
For any functions $g_{1}, g_{2}$ introduce the skew symmetric inner product:

$$
\left\langle g_{1}, g_{2}\right\rangle=\sum_{x_{1}, x_{2} \in \mathfrak{X}} \epsilon\left(x_{1}, x_{2}\right) g_{1}\left(x_{1}\right) g_{2}\left(x_{2}\right) f\left(x_{1}\right) f\left(x_{2}\right)
$$

and the monic skew symmetric orthogonal polynomials $p_{i}$ with respect to this inner product. (We assume that the weight $f$ is such that $\left.\left.\operatorname{det}||\left\langle x^{i}, x^{j}\right\rangle\right|_{i, j=1} ^{N} \neq 0\right)$. These skew symmetric orthogonal polynomials are constructed so that they satisfy the same orthogonality conditions as in Definition 3.5.3. but with respect to the skew symmetric inner product defined by equation (3.6.4). Wordfor-word repetition of arguments of the previous Section gives

Proposition 3.6.3. The normalization constant and the averages of characteristic polynomials must be of the same form for the discrete orthogonal and symplectic ensembles, up to the definition of the skew symmetric inner product. In particular we have

$$
\begin{gathered}
c_{N}^{(1)}=\prod_{i=0}^{N-1} h_{i}, \quad h_{i}=\left\langle p_{2 i}, p_{2 i+1}\right\rangle \\
p_{2 N}(\zeta)=\langle d(\zeta)\rangle_{\triangle_{2 N}^{(1)}(f)} \\
\left\langle d^{-1}(\zeta)\right\rangle_{\triangle_{2 N}^{(1)}(f)}=h_{N-1}^{-1}\left\langle p_{2 N-2}, R_{\zeta}\right\rangle \\
\left\langle\frac{d(\eta)}{d(\zeta)}\right\rangle_{\triangle_{2 N}^{(1)}(f)}=h_{N-1}^{-1}\left\langle\frac{1}{R_{\eta}(.)}\langle d(\eta) d(.)\rangle_{\triangle_{2 N-2}^{(1)}(f)}, \frac{R_{\zeta}(.)}{R_{\eta}(.)}\right\rangle \\
\left\langle\frac{1}{d(\eta) d(\zeta)}\right\rangle_{\triangle_{2 N}^{(1)}(f)}=h_{N-1}^{-1}\left\langle R_{\eta}(.)\left\langle\frac{d(.)}{d(\eta)}\right\rangle_{\triangle_{2 N-2}^{(1)}(f)} K_{N+1}^{C D, 1}(\zeta, \eta)\right. \\
\zeta-\eta \\
\left.R_{\eta}(.) R_{\zeta}(.)\right\rangle
\end{gathered}
$$

Here the Christoffel-Darboux kernel $K_{N+1}^{C D, 1}(\zeta, \eta)$ is given by formula (3.5.18), where the skew symmetric orthogonal polynomials $p_{i}$ are constructed with respect to inner product 3.6.4).

Define the Cauchy type transforms of the skew orthogonal polynomials by the same way as they were defined in the case of the discrete symplectic ensembles, see Definition 3.5.12. Then we obtain the following

Proposition 3.6.4. The averages of two characteristic polynomials with respect to the discrete orthogonal ensemble are Christoffel-Darboux type kernels constructed from the skew orthogonal polynomials associated with this ensemble, and their Cauchy type transforms:

- $\langle d(\zeta) d(\eta)\rangle_{\triangle_{2 N}^{(1)}(f)}=\frac{h_{N}}{\zeta-\eta} \sum_{i=0}^{N} \frac{p_{2 i+1}(\zeta) p_{2 i}(\eta)-p_{2 i}(\zeta) p_{2 i+1}(\eta)}{h_{i}}$

- $\left\langle\frac{d(\eta)}{d(\zeta)}\right\rangle_{\triangle_{2 N}^{(1)}(f)}=(\eta-\zeta) \sum_{i=0}^{N-1} \frac{p_{2 i+1}(\eta) h_{2 i}(\zeta)-p_{2 i}(\eta) h_{2 i+1}(\zeta)}{h_{i}}+1$

- $\left\langle\frac{1}{d(\eta) d(\zeta)}\right\rangle_{\triangle_{2 N}^{(1)}(f)}=\frac{1}{h_{N-1}} \frac{1}{\eta-\zeta}\left[\sum_{i=0}^{N-2} \frac{h_{2 i+1}(\eta) h_{2 i}(\zeta)-h_{2 i}(\eta) h_{2 i+1}(\zeta)}{h_{i}}+\left\langle R_{\eta}, R_{\zeta}\right\rangle\right]$

Proof. Straightforward computation of the skew symmetric products in the righthand sides of equations (3.6.8)-(3.6.10). 
3.7. Relation of ensembles. This Section discusses the relation between Pfaffian $L$-ensembles, discrete symplectic ensembles and the discrete orthogonal ensembles, introduced in Section 3.2 Section [3.5, and Section [3.6 respectively. Given $\mathfrak{X}$ consider the Pfaffian $L$-ensemble, defined by equations (3.2.1)-(3.2.6). Denote $\left|\mathfrak{X}_{-}\right|=2 M,\left|\mathfrak{X}_{+}\right|=2 N, M, N \in \mathbb{Z}_{>0}$, and assume that the weight $h$ is strictly positive on $\mathfrak{X}$. Given $h$ introduce the weights $f^{(4)}, f^{(1)}$ by

$$
\begin{aligned}
& f^{(4)}(x)= \begin{cases}\frac{h(x)}{\prod_{y \in \mathfrak{X}_{-}|x-y|},} & x \in \mathfrak{X}_{+} \\
\frac{1}{h(x) \prod_{y \in \mathfrak{X}_{-}, y \neq x}|x-y|}, & x \in \mathfrak{X}_{-}\end{cases} \\
& f^{(1)}(x)= \begin{cases}\frac{h(x)}{\prod_{y \in \mathfrak{X}_{+}|x-y|},} & x \in \mathfrak{X}_{-} \\
\frac{1}{h(x) \prod_{y \in \mathfrak{X}_{+}, y \neq x}|x-y|}, & x \in \mathfrak{X}_{+}\end{cases}
\end{aligned}
$$

Note that the only difference in the definition of $f^{(1)}$ and $f^{(4)}$ is the interchange of $\mathfrak{X}_{-}$and $\mathfrak{X}_{+}$. It follows from the two equations above that

$$
f^{(1)}(x) \cdot f^{(4)}(x)=\prod_{y \in \mathfrak{X}, y \neq x}|x-y|^{-1}, x \in \mathfrak{X}
$$

Theorem 3.7.1. The Pfaffian L-ensemble with the weight $h$ defined by equations (3.2.1)-(3.2.6), the discrete symplectic ensemble with the weight $f^{(4)}$ defined by equation (3.5.1), and the discrete orthogonal ensemble with the weight $f^{(1)}$, defined by equation (3.6.1) are equivalent in the following sense:

a) There is a bijection between the sets $\operatorname{Conf}^{L}(\mathfrak{X})$ and $\operatorname{Conf}_{2 M}^{(4)}(\mathfrak{X})$ defined by the particle-hole involution on $\mathfrak{X}_{+}$. Under this bijection the probability distribution for the Pfaffian L-ensemble, equation (3.2.7), turns into the probability distribution for the discrete symplectic ensemble, equation (3.5.1).

b) There is a bijection between the sets $\operatorname{Conf}^{L}(\mathfrak{X})$ and $\operatorname{Conf}_{2 N}^{(1)}(\mathfrak{X})$ defined by the particle-hole involution on $\mathfrak{X}_{-}$. Under this bijection the probability distribution for the Pfaffian L-ensemble, equation (3.2.7), turns into the probability distribution for the discrete orthogonal ensemble, equation (3.6.1).

c) There is a bijection between the sets $\operatorname{Con} f_{2 N}^{(1)}(\mathfrak{X})$ and $\operatorname{Con} f_{2 M}^{(4)}(\mathfrak{X})$ defined by the particle-hole involution on the whole set $\mathfrak{X}$. Under this bijection the probability distribution for the discrete orthogonal ensemble, equation (3.6.1) turns into the probability distribution for the discrete symplectic ensemble, 3.5.1.

In other words, the probability spaces defined by $\operatorname{Conf}^{L}(\mathfrak{X}), \operatorname{Conf}_{2 M}^{(4)}(\mathfrak{X}), \operatorname{Conf}_{2 N}^{(1)}(\mathfrak{X})$ with the associated probability distributions are isomorphic.

Proof. Given $\operatorname{Con}^{L}(\mathfrak{X})$, let $X \in \operatorname{Con}_{2 N}^{L}(\mathfrak{X})$, and $\tilde{X}$ be the point configuration associated with $X$, see Section 3.2 . Consider the particle-hole involution on $\mathfrak{X}_{-}$. Under this involution the configuration $\tilde{X}$ turns into the configuration $Y$ related with the configuration $\tilde{X}$ by the formula:

$$
Y=Y_{-} \sqcup Y_{+}, \quad Y_{-}=\mathfrak{X}_{-} \backslash \tilde{X}_{-}, \quad Y_{+}=\tilde{X}_{+} .
$$

The key observation is that the configuration $Y$ is in $\operatorname{Conf}_{2 M}^{(4)}(\mathfrak{X})$. It can be immediately seen from the definition of $\operatorname{Con}_{2 M}^{(4)}(\mathfrak{X})$. Clearly, the particle-hole involution on $\mathfrak{X}_{-}$is a bijection between $\operatorname{Conf}^{L}(\mathfrak{X})$ and $\operatorname{Conf}_{2 M}^{(4)}(\mathfrak{X})$. In order to prove a), it remains to show that the probability distribution on $\operatorname{Conf}^{L}(\mathfrak{X})$, equation (3.2.7), turns into the probability distribution on $\operatorname{Con} f_{2 M}^{(4)}(\mathfrak{X})$, equation (3.5.1), provided the weights are related by formula (3.7.1). This is a consequence of two 
facts. The first one is $|V(Y)|$ can be rewritten as

$$
|V(Y)|=\mathrm{const} \frac{V\left(\tilde{X}_{+}\right) V\left(\tilde{X}_{-}\right)}{\prod\left(\tilde{X}_{+} ; \tilde{X}_{-}\right)} \frac{\prod_{x \in \tilde{X}_{+}} \prod_{y \in \mathfrak{X}_{-}}|x-y|}{\prod_{x \in \tilde{X}_{-}} \prod_{y \in \mathfrak{X}_{-}, y \neq x}|x-y|}, \quad \text { const }=\left|V\left(\mathfrak{X}_{-}\right)\right|
$$

This follows from the relation $Y$ and $\tilde{X}$ (equation (3.7.4) ). The second fact is the relation:

$$
f(Y)=\text { const } \frac{f\left(\tilde{X}_{+}\right)}{f\left(\tilde{X}_{-}\right)}, \quad \text { const }=f(\mathfrak{X})
$$

We then see that the righthand side of equation (3.2.7) is identical to that of equation (3.5.1), if $h$ and $f$ are related by equation (3.7.1). Thus we have proved a).

The proof of $\mathrm{b}$ ) is constructed in the same way. The configuration $Y$ obtained by the particle-hole involution on $\mathfrak{X}_{-}$from $\tilde{X}$ is

$$
Y=Y_{-} \sqcup Y_{+}, \quad Y_{-}=\tilde{X}_{-}, \quad Y_{+}=\mathfrak{X}_{+} \backslash \tilde{X}_{+} .
$$

Instead of formula (3.7.5) we obtain

$$
|V(Y)|=\operatorname{const} \frac{V\left(\tilde{X}_{+}\right) V\left(\tilde{X}_{-}\right)}{\prod\left(\tilde{X}_{+} ; \tilde{X}_{-}\right)} \frac{\prod_{x \in \tilde{X}_{-}} \prod_{y \in \mathfrak{X}_{+}}|x-y|}{\prod_{x \in \tilde{X}_{+}} \prod_{y \in \mathfrak{X}_{+}, y \neq x}|x-y|}, \quad \text { const }=\left|V\left(\mathfrak{X}_{+}\right)\right|
$$

which leads (together with the relation of the weights $h$ and $f^{(1)}$, equation (3.7.2) ) to the equivalence of the probability distributions on $\operatorname{Conf}^{L}(\mathfrak{X})$ and $\operatorname{Con} f_{2 N}^{(1)}(\mathfrak{X})$.

The statement c) obviously follows from a) and b). However, it is easy to give an independent argument. Let $X \in \operatorname{Conf}_{2 M}^{(4)}(\mathfrak{X})$. Then the particle-hole involution on $\mathfrak{X}$ gives a configuration $Y$ which is in $\operatorname{Con} f_{2 N}^{(1)}(\mathfrak{X})$. For $X$ and $Y$ related by the particle-hole involution we obtain

$$
|V(X)|=\text { const }|V(Y)| \prod_{x \in X} \prod_{y \in \mathfrak{X}, y \neq x}|x-y|, \quad \text { const }=|V(\mathfrak{X})|^{-1}
$$

This expression (together with the relation of weights $f^{(1)}$ and $f^{(4)}$, equation (3.7.3)) leads to the equivalence of the discrete symplectic and the discrete orthogonal ensembles.

Assume that the Pfaffian $L$-ensemble is given, and denote by $\triangle_{2 M}\left(f^{(4)}\right)$ and $\triangle_{2 N}\left(f^{(1)}\right)$ the equivalent discrete symplectic and orthogonal ensembles obtained from the Pfaffian $L$-ensemble by the particle-hole involution. With the Pfaffian $L$-ensemble we can construct a new ensemble, Pfaffian $\hat{L}$-ensemble, by new splitting of $\mathfrak{X}, \mathfrak{X}=\hat{X}_{-} \sqcup \hat{X}_{+},\left|\hat{\mathfrak{X}}_{+}\right|=2 N+2 S,\left|\hat{\mathfrak{X}}_{-}\right|=2 M-2 S$, see Section 3.3 . This Pfaffian $\hat{L}$-ensemble induces a discrete symplectic ensemble of $2(M-S)$ point configurations, and a discrete orthogonal ensemble of $2(N+S)$ point configurations. The first one is obtained by the particle-hole involution on $\hat{\mathfrak{X}}_{-}$, and the second one is obtained by the particle-hole involution on $\hat{\mathfrak{X}}_{+}$. Let $\hat{h}$ denotes the weight of the Pfaffian $\hat{L}$-ensemble. Recall that it is given in terms of the weight $h$ of the original Pfaffian $L$-ensemble by formula (3.3.6). The Theorem above says that the Pfaffian $\hat{L}$ ensemble with weight $\hat{h}$, the discrete symplectic ensemble $\triangle_{2(M-S)}\left(\hat{f}^{(4)}\right)$, and the discrete orthogonal ensemble $\triangle_{2(N+S)}\left(\hat{f}^{(1)}\right)$ are equivalent provided the weights are related by formulae (3.7.1)- (3.7.2), with $\mathfrak{X}_{ \pm} \rightarrow \hat{\mathfrak{X}}_{ \pm}$.

Proposition 3.7.2. The discrete symplectic ensembles $\triangle_{2 M}\left(f^{(4)}\right)$ and $\triangle_{2(M-S)}\left(\hat{f}^{(4)}\right)$ have the same weight: $f^{(4)} \equiv \hat{f}^{(4)}$. The discrete orthogonal ensembles $\triangle_{2 N}\left(f^{(1)}\right)$ and $\triangle_{2 N+2 S}\left(\hat{f}^{(1)}\right)$ have the same weight: $f^{(1)} \equiv \hat{f}^{(1)}$.

Proof. Application of the same arguments as in the case of the determinantal point ensembles, see Proposition 2.6.2. 
3.8. Averages of characteristic polynomials: symplectic and orthogonal ensembles. Our goal now is to derive averages of product and ratios for symplectic and orthogonal ensembles. We will use the relation of these ensembles with the Pfaffian $L$-ensembles discussed in the previous Section, and Proposition 3.4.1. We begin with symplectic ensembles.

Given equation (3.4.4) let us rewrite its righthand side in terms of the discrete symplectic ensembles. We proceed as in the determinantal case, see Section 2.7

Proposition 3.8.1. The constant in equation (3.4.4) is the ratio of the normalization constants for the discrete symplectic ensembles of $2 N-2 S$ particles and of $2 N$ particles,

$$
\frac{\operatorname{Pf}(J+\hat{L})}{\operatorname{Pf}(J+L)} h\left(\mathfrak{X}_{0}\right)\left|V\left(\mathfrak{X}_{0}\right)\right|=\frac{c_{N-S}^{(4)}}{c_{N}^{(4)}}
$$

Proof. There are two Pfaffian ensembles involved in Proposition 3.4.1. The first one, the Pfaffian $L$-ensemble, is defined with respect to the splitting $\mathfrak{X}=\mathfrak{X}_{-} \sqcup \mathfrak{X}_{+}$, and the second one, the Pfaffian $\hat{L}$-ensemble, is defined with respect to the splitting $\hat{\mathfrak{X}}=\hat{\mathfrak{X}}_{-} \sqcup \hat{\mathfrak{X}}_{+}$. The expression $[\operatorname{Pf}(J+L)]^{-1}$ is the probability of the empty configuration for the first Pfaffian ensemble, and $[\operatorname{Pf}(J+\hat{L})]^{-1}$ is the probability of the empty configuration for the second Pfaffian ensemble.

By the particle-hole involution on $\mathfrak{X}_{-}$and $\hat{\mathfrak{X}}_{-}$construct the equivalent discrete symplectic ensembles. Then the empty configurations of the Pfaffian ensembles turn into certain configurations of these symplectic ensembles. It is not hard to see that these configurations are $\mathfrak{X}_{-}$and $\hat{\mathfrak{X}}_{-}$. Equate the probabilities:

$$
\frac{1}{\operatorname{Pf}(J+L)}=\frac{1}{c_{N}^{(4)}} f\left(\mathfrak{X}_{-}\right)\left|V\left(\mathfrak{X}_{-}\right)\right|, \quad \frac{1}{\operatorname{Pf}(J+\hat{L})}=\frac{1}{c_{N-S}^{(4)}} f\left(\hat{\mathfrak{X}}_{-}\right)\left|V\left(\hat{\mathfrak{X}}_{-}\right)\right|
$$

These equations, together with the relation between weights, equation (3.7.1), give formula (3.8.1).

Theorem 3.8.2. For any integer $N \geq 1$ take an integer $S$ such that $2-2 N \leq 2 S \leq|\mathfrak{X}|-2-2 N$, complex numbers $\alpha=\left(\alpha_{-}, \alpha_{+}\right), \alpha_{-}=\left(\alpha_{1}^{-}, \ldots \alpha_{m}^{-}\right), \alpha_{+}=\left(\alpha_{1}^{+}, \ldots, \alpha_{k}^{+}\right)$such that $\left|\alpha_{+}\right|-\left|\alpha_{-}\right|=$ $k-m=2 S$, in each set $\alpha_{ \pm}$the numbers are pairwise distinct, and the set $\alpha$ does not intersect $\mathfrak{X}$. Then the average of products and ratios of characteristic polynomials with respect to the discrete symplectic ensemble $\triangle_{2 N}^{(4)}(f)$ is given by the formula:

$$
\left\langle\frac{\prod_{i=1}^{k} d\left(\alpha_{i}^{+}\right)}{\prod_{i=1}^{m} d\left(\alpha_{i}^{-}\right)}\right\rangle_{\triangle_{2 N}^{(4)}(f)}=\frac{c_{N+S}^{(4)}}{c_{N}^{(4)}} \frac{\prod\left(\alpha_{-} ; \alpha_{+}\right)}{V\left(\alpha_{-}\right) V\left(\alpha_{+}\right)} \operatorname{Pf}\left[W_{N}^{(4)}(\alpha \mid \alpha)\right]
$$

where the kernel function $W_{N}^{(4)}$ is defined by

$$
\begin{aligned}
& \text { - } W_{N}^{(4)}\left(\alpha_{i}^{+}, \alpha_{j}^{+}\right)=h_{N+S-1}^{-1}\left(\alpha_{i}^{+}-\alpha_{j}^{+}\right)\left\langle d\left(\alpha_{i}^{+}\right) d\left(\alpha_{j}^{+}\right)\right\rangle_{\triangle_{2 N+2 S-2}^{(4)}(f)} \\
& \text { - } W_{N}^{(4)}\left(\alpha_{i}^{-}, \alpha_{j}^{+}\right)=-W_{N}^{(4)}\left(\alpha_{j}^{+}, \alpha_{i}^{-}\right)=\frac{1}{\alpha_{i}^{-}-\alpha_{j}^{+}}\left\langle\frac{d\left(\alpha_{j}^{+}\right)}{d\left(\alpha_{i}^{-}\right)}\right\rangle_{\triangle_{2 N+2 S}^{(4)}(f)} \\
& \text { - } W_{N}^{(4)}\left(\alpha_{i}^{-}, \alpha_{j}^{-}\right)=h_{N+S}\left(\alpha_{i}^{-}-\alpha_{j}^{-}\right)\left\langle\frac{1}{d\left(\alpha_{i}^{-}\right) d\left(\alpha_{j}^{-}\right)}\right\rangle_{\triangle_{2 N+2 S+2}^{(4)}(f)}
\end{aligned}
$$

Proof. Assume first that $S \geq 0$. We use equation (3.4.4). Denote by $\triangle_{2 N-2 S}^{(4)}\left(f^{(4)}\right)$ the discrete symplectic ensemble obtained by the particle-hole involution on $\hat{\mathfrak{X}}$ from the Pfaffian $\hat{L}$-ensemble involved in equation (3.4.4). We want to rewrite the righthand side of equation (3.4.4) in terms of $\triangle_{2 N-2 S}^{(4)}\left(f^{(4)}\right) . \triangle_{2 N-2 S}^{(4)}\left(f^{(4)}\right)$ and the Pfaffian $\hat{L}$-ensemble involved in equation (3.4.4) are equivalent, the corresponding probability measures are equal to each other. We only need to express 
the functions $E(.,$.$) in terms of characteristic polynomials associated with \triangle_{2 N-2 S}^{(4)}\left(f^{(4)}\right)$. For any finite set $\alpha, \alpha \cap \mathfrak{X}=\varnothing$, we find

$$
E(\alpha, X) \cdot \prod\left(\alpha ; \hat{\mathfrak{X}}_{-}\right)=\prod(\alpha ; Y) \equiv \prod_{i} d\left(\alpha_{i}\right)
$$

where the configurations $X \in \operatorname{Conf}^{\hat{L}}(\mathfrak{X})$ and $Y \in \operatorname{Conf}_{2 N-2 S}^{(4)}(\mathfrak{X})$ are related by the particlehole involution. This immediately follows from the definition of $E(.,$.$) , equation (2.4.2). This$ observation and Proposition 3.8.1 give

$$
\operatorname{Pf}\left[K\left(\alpha^{\prime} \mid \alpha^{\prime}\right)\right]=\frac{c_{N-S}^{(4)}}{c_{N}^{(4)}} h(\alpha) \frac{V\left(\alpha_{-}\right) V\left(\alpha_{+}\right)}{\prod\left(\alpha_{-} ; \alpha_{+}\right)}\left[\frac{\prod\left(\alpha_{+} ; \mathfrak{X}_{-}\right)}{\prod\left(\alpha^{-} ; \mathfrak{X}_{-}\right)}\right]\left\langle\frac{\prod_{i=1}^{k} d\left(\alpha_{i}^{+}\right)}{\prod_{i=1}^{m} d\left(\alpha_{i}^{-}\right)}\right\rangle_{\triangle_{2 N-2 S}^{(4)}(f)}
$$

Considering particular cases corresponding to $k=0, m=2 ; k=1, m=1, k=2, m=0$ determine the kernel function $K$ in this equation, and obtain the formula stated in the Theorem.

The case $S \leq 0$ corresponds to the splitting

$$
\mathfrak{X}=\hat{\mathfrak{X}}_{-} \sqcup \hat{\mathfrak{X}}_{+}, \quad \hat{\mathfrak{X}}_{-}=\mathfrak{X}_{-} \sqcup \mathfrak{X}_{0}, \quad \hat{\mathfrak{X}}_{+}=\mathfrak{X}_{+} \backslash \mathfrak{X}_{0}
$$

and is considered in the same way.

Applying the same arguments we prove the corresponding result for the discrete orthogonal ensembles.

Theorem 3.8.3. For any integer $N \geq 1$ take an integer $S$ such that $2 N+2-2|\mathfrak{X}| \leq 2 S \leq 2 N-2$, complex numbers $\alpha=\left(\alpha_{-}, \alpha_{+}\right), \alpha_{-}=\left(\alpha_{1}^{-}, \ldots \alpha_{m}^{-}\right), \alpha_{+}=\left(\alpha_{1}^{+}, \ldots, \alpha_{k}^{+}\right)$such that $\left|\alpha_{+}\right|-\left|\alpha_{-}\right|=$ $k-m=2 S$, in each set $\alpha_{ \pm}$the numbers are pairwise distinct, and the set $\alpha$ does not intersect $\mathfrak{X}$. Then the average of products and ratios of characteristic polynomials with respect to the discrete orthogonal ensemble $\triangle_{2 N}^{(1)}(f)$ is given by the formula:

$$
\left\langle\frac{\prod_{i=1}^{m} d\left(\alpha_{i}^{-}\right)}{\prod_{i=1}^{k} d\left(\alpha_{i}^{+}\right)}\right\rangle_{\triangle_{2 N}^{(1)}(f)}=\frac{c_{N-S}^{(1)}}{c_{N}^{(1)}} \frac{\prod\left(\alpha_{-} ; \alpha_{+}\right)}{V\left(\alpha_{-}\right) V\left(\alpha_{+}\right)} \operatorname{Pf}\left[W_{N}^{(1)}(\alpha \mid \alpha)\right]
$$

where the kernel function $W_{N}^{(1)}$ is defined by

$$
\begin{aligned}
& \text { - } W_{N}^{(1)}\left(\alpha_{i}^{+}, \alpha_{j}^{+}\right)=h_{N-S}\left(\alpha_{i}^{+}-\alpha_{j}^{+}\right)\left\langle\frac{1}{d\left(\alpha_{i}^{+}\right) d\left(\alpha_{j}^{+}\right)}\right\rangle_{\triangle_{2 N-2 S-2}^{(1)}(f)} \\
& \text { - } W_{N}^{(1)}\left(\alpha_{i}^{-}, \alpha_{j}^{+}\right)=\frac{1}{\alpha_{i}^{-}-\alpha_{j}^{+}}\left\langle\frac{d\left(\alpha_{i}^{-}\right)}{d\left(\alpha_{j}^{+}\right)}\right\rangle_{\triangle_{2 N-2 S}^{(1)}(f)} \\
& \text { - } W_{N}^{(1)}\left(\alpha_{i}^{-}, \alpha_{j}^{-}\right)=\frac{1}{h_{N-S-1}}\left(\alpha_{i}^{-}-\alpha_{j}^{-}\right)\left\langle d\left(\alpha_{i}^{-}\right) d\left(\alpha_{j}^{-}\right)\right\rangle_{\triangle_{2 N-2 S+2}^{(1)}(f)}
\end{aligned}
$$

3.9. Computation of the $m$-point correlation function from the averages of characteristic polynomials. The aim of this Section is to illustrate the convenience and the generality of the averages of the characteristic polynomials. Similary to the case of the polynomial ensembles we extract the standard $m$-point correlation function from this averages.

Proposition 3.9.1. The m-point correlation function of the discrete orthogonal ensemble $\triangle_{2 N}^{(1)}(f)$ is given by the formula:

$$
\varrho_{m}\left(z_{1}, \ldots, z_{m}\right)=(-)^{\frac{m(m-1)}{2}} \operatorname{Pf}\left[\begin{array}{cc}
{\left[D\left(z_{i}, z_{j}\right)\right]_{i, j=1}^{m}} & {\left[S\left(z_{i}, z_{j}\right)\right]_{i, j=1}^{m}} \\
{\left[-S\left(z_{j}, z_{i}\right)\right]_{i, j=1}^{m}} & {\left[I\left(z_{i}, z_{j}\right)\right]_{i, j=1}^{m}}
\end{array}\right]
$$

where

$$
\text { - } D(\zeta, \eta)=\frac{1}{h_{N-1}}(\zeta-\eta)\langle d(\zeta) d(\eta)\rangle_{\triangle_{2 N-2}^{(1)}(f)}
$$


$\begin{aligned} \text { - } I(\zeta, \eta) & =\operatorname{Res}_{\substack{x=\zeta \\ y=\eta}}\left[h_{N}(x-y)\left\langle\frac{1}{d(x) d(y)}\right\rangle_{\triangle_{2 N+2}^{(1)}(f)}\right] \\ \text { - } S(\zeta, \eta) & = \begin{cases}\operatorname{Res}_{y=\eta}\left[\frac{1}{\zeta-y}\left\langle\frac{d(\zeta)}{d(y)}\right\rangle_{\triangle_{2 N}^{(1)}(f)}\right], \quad \zeta \neq \eta \\ \operatorname{Res}_{y=\eta}\left[\left.\frac{d}{d x}\right|_{x=y}\left\langle\frac{d(x)}{d(y)}\right\rangle_{\triangle_{2 N}^{(1)}(f)}\right], \quad \zeta=\eta\end{cases} \end{aligned}$

Proof. Set $\alpha_{-}=\left(x_{1}, \ldots, x_{m}\right), \alpha_{+}=\left(y_{1}, \ldots, y_{m}\right)$ in Theorem 3.8.3. Use the formula for the Cauchy determinant to rewrite equation (3.8.4) as

$$
\left\langle\operatorname{det}\left[\frac{1}{x_{i}-y_{j}} \frac{d\left(x_{i}\right)}{d\left(y_{j}\right)}\right]_{i, j=1}^{m}\right\rangle_{\triangle_{2 N}^{(1)}(f)}=(-)^{\frac{m(m-1)}{2}} \operatorname{Pf}\left[W_{N}^{(1)}(x, y \mid x, y)\right]
$$

Multiply the left-hand side of this equation by the product $\left(x_{1}-y_{1}\right) \ldots\left(x_{m}-y_{m}\right)$. Differentiate the obtained expression by $x_{1}, \ldots x_{m}$ and take parameters $x_{i}$ to be equal to the parameters $y_{i}$. We then obtain some function of $y_{1}, \ldots, y_{m}$. By taking residues at points $y_{1}=z_{1}, \ldots, y_{m}=z_{m}$ from this function we get $\varrho_{m}\left(z_{1}, \ldots, z_{m}\right)$ as we have learned previously in the determinantal case, Proposition 2.8.1.

We want to understand what happens with the righthand side under the same transformations. Decompose the Pfaffian into the sum over permutations. Now we multiply this sum by $\prod_{i=1}^{m}\left(x_{i}-y_{i}\right)$, differentiate and substitute $x_{i}=y_{i}, i=\overline{1, m}$. We observe that all terms of this sum which do not include $W_{N}^{(1)}\left(x_{l}, y_{l}\right)$ remain unchanged, we only replace $x_{i}$ by $y_{i}$. As for the elements like $W_{N}^{(1)}\left(x_{l}, y_{l}\right)$ they turn into the elements

$$
\left.W_{N}^{(1)}\left(x_{l}, y_{l}\right) \rightarrow \frac{d}{d x_{l}}\left\langle\frac{d\left(x_{l}\right)}{d\left(y_{l}\right)}\right\rangle_{\triangle_{2 N}^{(1)}(f)}\right|_{x_{l}=y_{l}}
$$

Thus the obtained sum is equal to

$$
\operatorname{Pf}\left[\begin{array}{cc}
{\left[\tilde{D}\left(y_{i}, y_{j}\right)\right]_{i, j=1}^{m}} & {\left[\tilde{S}\left(y_{i}, y_{j}\right)\right]_{i, j=1}^{m}} \\
{\left[-\tilde{S}\left(y_{j}, y_{i}\right)\right]_{i, j=1}^{m}} & {\left[\tilde{I}\left(y_{i}, y_{j}\right)\right]_{i, j=1}^{m}}
\end{array}\right]
$$

where

- $\tilde{D}\left(y_{i}, y_{j}\right)=\frac{1}{h_{N-1}}\left(y_{i}-y_{j}\right)\left\langle d\left(y_{i}\right) d\left(y_{j}\right)\right\rangle_{\triangle_{2 N-2}^{(1)}(f)}$

- $\tilde{I}\left(y_{i}, y_{j}\right)=h_{N}\left(y_{i}-y_{j}\right)\left\langle\frac{1}{d\left(y_{i}\right) d\left(y_{j}\right)}\right\rangle_{\triangle_{2 N+2}^{(1)}(f)}$

- $\tilde{S}\left(y_{i}, y_{j}\right)= \begin{cases}\frac{1}{y_{i}-y_{j}}\left\langle\frac{d\left(y_{i}\right)}{d\left(y_{j}\right)}\right\rangle_{\triangle_{2 N}^{(1)}(f)}, \quad i \neq j \\ \left.\frac{d}{d x}\right|_{x=y_{i}}\left\langle\frac{d(x)}{d\left(y_{i}\right)}\right\rangle_{\triangle_{2 N}^{(1)}(f)}, \quad i=j\end{cases}$

It remains to take the residues. Rewrite once again the Pfaffian as a sum over permutations. Taking into account the definition of the Pfaffian it is not hard to see that after taking $\underset{y_{1}=z_{1}}{\operatorname{Res}}, \ldots \underset{y_{m}=z_{m}}{\operatorname{Res}}$ the two point functions $\tilde{D}, \tilde{I}$, and $\tilde{S}$ turn into $D, S$ and $I$.

Proposition 3.9.2. The correlation functions of the discrete symplectic ensemble are given by the formulas of Proposition 3.9.1 with averages over $\triangle^{(1)}$ replaced by similar averages over $\triangle^{(4)}$. 
Corollary 3.9.3. The m-point correlation function for the discrete orthogonal ensemble $\triangle_{2 N}^{(1)}(f)$ is given by the quaternion determinant Tdet of the $2 \times 2$ matrix valued kernel $\sigma\left(z_{i}, z_{j}\right), i, j=\overline{1, m}$,

$$
\varrho_{m}\left(z_{1}, \ldots, z_{m}\right)=\operatorname{Tdet} \sigma\left(z_{i}, z_{j}\right)_{i, j=1}^{m}
$$

where

$$
\sigma\left(z_{i}, z_{j}\right)=\left(\begin{array}{cc}
-S\left(z_{i}, z_{j}\right) & I\left(z_{i}, z_{j}\right) \\
-D\left(z_{i}, z_{j}\right) & -S\left(z_{j}, z_{i}\right)
\end{array}\right)
$$

the two-point functions $D, I, S$ are given by

- $D\left(z_{i}, z_{j}\right)=\sum_{l=0}^{N-1} \frac{p_{2 l+1}\left(z_{i}\right) p_{2 l}\left(z_{j}\right)-p_{2 l}\left(z_{i}\right) p_{2 l+1}\left(z_{j}\right)}{h_{l}}$

- $I\left(z_{i}, z_{j}\right)=f\left(z_{i}\right) f\left(z_{j}\right)\left[\sum_{l=0}^{N-1} \frac{q_{2 l+1}\left(z_{i}\right) q_{2 l}\left(z_{j}\right)-q_{2 l}\left(z_{i}\right) q_{2 l+1}\left(z_{j}\right)}{h_{l}}+\epsilon\left(z_{i}, z_{j}\right)\right]$

- $S\left(z_{i}, z_{j}\right)=f\left(z_{j}\right)\left[\sum_{l=0}^{N-1} \frac{p_{2 l+1}\left(z_{i}\right) q_{2 l}\left(z_{j}\right)-p_{2 l}\left(z_{i}\right) q_{2 l+1}\left(z_{j}\right)}{h_{l}}\right]$

and the functions $q_{k}(z)$ are defined by

$$
\underset{\zeta=z}{\operatorname{Res}} h_{k}(\zeta)=f(z) q_{k}(z)
$$

Remark 3.9.4. With the specific choice $\epsilon(x, y)=\epsilon(x-y)$ on a one dimensional lattice,

$$
\epsilon(x, y)= \begin{cases}-1 / 2, & x>y \\ 0, & x=y \\ 1 / 2, & x<y\end{cases}
$$

the formula for the $m$-point correlation function is reduced to that of Nagao and Forrester [46].

Proof. Compute the two point functions $D, I, S$ in Proposition 3.9.1. We use the formulas which express averages of two characteristic polynomials as the Christoffel-Darbyoux type kernels constructed from the skew orthogonal polynomials and their Cauchy type transforms, Proposition 3.6.4. Taking into account equation (3.9.4) we immediately obtain the expressions for $D, I, S$ stated in the Corollary. Thus we have

$$
\varrho_{m}\left(z_{1}, \ldots, z_{m}\right)=(-)^{\frac{m(m-1)}{2}} \operatorname{Pf}\left[\begin{array}{cc}
{\left[D\left(z_{i}, z_{j}\right)\right]_{i, j=1}^{m}} & {\left[S\left(z_{i}, z_{j}\right)\right]_{i, j=1}^{m}} \\
{\left[-S\left(z_{j}, z_{i}\right)\right]_{i, j=1}^{m}} & {\left[I\left(z_{i}, z_{j}\right)\right]_{i, j=1}^{m}}
\end{array}\right]
$$

It remains to rewrite the righthand side of this expression as the quaternion determinant of $2 \times 2$ matrix valued kernel. This is achieved in two steps. The first step is to observe that

$$
\operatorname{Pf}\left[W\left(x_{1}, \ldots, x_{m} ; y_{1}, \ldots, y_{m} \mid x_{1}, \ldots, x_{m} ; y_{1}, \ldots, y_{m}\right)\right]=(-)^{\frac{m(m-1)}{2}} \operatorname{Pf}\left[w\left(z_{i}, z_{j}\right)\right]_{i, j=1}^{m}
$$

where

$$
w\left(z_{i}, z_{j}\right)=\left[\begin{array}{ll}
W\left(x_{i}, x_{j}\right) & W\left(x_{i}, y_{j}\right) \\
W\left(y_{i}, x_{j}\right) & W\left(y_{i}, y_{j}\right)
\end{array}\right]
$$

The second step is to apply the formula

$$
\operatorname{Pf}[Z Q]=\operatorname{Tdet} Q, \quad Z=\left(\begin{array}{cccc}
J & 0 & \ldots & 0 \\
0 & J & \ldots & 0 \\
\vdots & & \ddots & \\
0 & 0 & \ldots & J
\end{array}\right)
$$

where $Q$ is a $2 \times 2$ block antisymmetric matrix, and $J=\left(\begin{array}{cc}0 & 1 \\ -1 & 0\end{array}\right)$

Remark 3.9.5. The statement similar to Corollary 3.9 .3 holds for the discrete symplectic ensembles as well. The proof is just the same. 


\section{Continuous Limit}

4.1. Exact formula for unitary $(\beta=2)$ ensembles. Let $\mu$ be a positive measure on $\mathbb{R}$ with finite moments: $\int_{\mathbb{R}}|x|^{n} \mu(d x)<\infty$ for any $n \geq 0$, such that

$$
C_{N}^{(\beta)}:=\frac{1}{N !} \int_{\mathbb{R}^{N}} \prod_{1 \leq i<j \leq N}\left|x_{i}-x_{j}\right|^{\beta} \mu\left(d x_{1}\right) \cdots \mu\left(d x_{N}\right) \neq 0
$$

for any $N \geq 1$ and some (equivalently, all) $\beta>0$. Denote by $\triangle_{N}^{(\beta)}$ the probability measure on $\mathbb{R}^{N}$ given by

$$
p_{N}^{(\beta)}\left(d x_{1}, \ldots, d x_{N}\right)=\frac{1}{N ! C_{N}^{(\beta)}} \prod_{1 \leq i<j \leq N}\left|x_{i}-x_{j}\right|^{\beta} \mu\left(d x_{1}\right) \otimes \cdots \otimes \mu\left(d x_{N}\right) .
$$

For any symmetric function $g: \mathbb{R}^{N} \rightarrow \mathbb{C}$ set

$$
\langle g\rangle_{\triangle_{N}^{(\beta)}}:=\int_{\mathbb{R}^{N}} g\left(x_{1}, \ldots, x_{N}\right) p_{N}^{(\beta)}\left(d x_{1}, \ldots, d x_{N}\right)
$$

provided that the integral converges. For $\zeta \in \mathbb{C}$ set

$$
D(\zeta)=\prod_{i \geq 1}\left(\zeta-x_{i}\right)
$$

The number of factors in such products will always be clear from the context. Recall that for finite sets $A=\left\{a_{1}, \ldots, a_{p}\right\}$ and $B=\left\{b_{1}, \ldots, b_{q}\right\}$ we use the notation $\prod(A ; B)$ to denote the product of pairwise differences

$$
\prod(A ; B)=\prod_{i=1}^{p} \prod_{j=1}^{q}\left(a_{i}-b_{j}\right)
$$

and $V(A)$ stands for the Vandermonde determinant $\prod_{1 \leq i<j \leq p}\left(a_{i}-a_{j}\right)$ (note that we use an ordering of $a_{i}$ 's here).

Theorem 4.1.1. (Case $\beta=2$ ) For any integers $N \geq 1$ and $S>1-N$, and finite sets of mutually distinct complex numbers

$$
\begin{aligned}
\alpha^{-}=\left\{\alpha_{1}^{-}, \ldots, \alpha_{m_{1}}^{-}\right\}, & \alpha^{+}=\left\{\alpha_{1}^{+}, \ldots, \alpha_{k_{1}}^{-}\right\}, \\
\beta^{-}=\left\{\beta_{1}^{-}, \ldots, \beta_{m_{2}}^{-}\right\}, & \beta^{+}=\left\{\beta_{1}^{+}, \ldots, \beta_{k_{2}}^{+}\right\},
\end{aligned}
$$

with $\left|\alpha^{-}\right|-\left|\alpha^{+}\right|=\left|\beta^{-}\right|-\left|\beta^{+}\right|=S$, such that

$$
\alpha^{-} \cap \alpha^{+}=\varnothing, \quad \beta^{-} \cap \beta^{+}=\varnothing, \quad \alpha^{+} \cap \mathbb{R}=\varnothing, \quad \beta^{+} \cap \mathbb{R}=\varnothing,
$$

one has

$$
\begin{aligned}
& \left\langle\frac{\prod_{i=1}^{m_{1}} D\left(\alpha_{i}^{-}\right) \prod_{i=1}^{m_{2}} D\left(\beta_{i}^{-}\right)}{\prod_{j=1}^{k_{1}} D\left(\alpha_{j}^{+}\right) \prod_{j=1}^{k_{2}} D\left(\beta_{j}^{+}\right)}\right\rangle_{\triangle_{N}^{(2)}}=(-1)^{\frac{1}{2}\left[\left(\left|\alpha^{-}\right|+\left|\beta^{-}\right|\right)^{2}+\left|\beta^{-}\right|-\left|\alpha^{-}\right|\right]} \\
& \times \frac{C_{N+S}^{(2)}}{C_{N}^{(2)}} \frac{\prod\left(\alpha^{-} ; \alpha^{+}\right) \prod\left(\beta^{-} ; \beta^{+}\right)}{V\left(\alpha^{-}\right) V\left(\alpha^{+}\right) V\left(\beta^{-}\right) V\left(\beta^{+}\right)} \operatorname{det}\left[W_{N}^{(2)}\left(\alpha^{-}, \beta^{+} \mid \beta^{-}, \alpha^{+}\right)\right] .
\end{aligned}
$$


Here $W_{N}^{(2)}\left(\alpha^{-}, \beta^{+} \mid \beta^{-}, \alpha^{+}\right)$is a matrix with rows parameterized by elements of $\alpha^{-}$and $\beta^{+}$, columns parameterized by elements of $\beta^{-}$and $\alpha^{+}$, and with matrix elements

$$
\begin{aligned}
& W_{N}^{(2)}\left(\alpha_{i}^{-}, \beta_{j}^{-}\right)=\frac{C_{N+S-1}^{(2)}}{C_{N+S}^{(2)}}\left\langle D\left(\alpha_{i}^{-}\right) D\left(\beta_{j}^{-}\right)\right\rangle_{\triangle_{N+S-1}^{(2)}} \\
& W_{N}^{(2)}\left(\alpha_{i}^{-}, \alpha_{j}^{+}\right)=\frac{1}{\alpha_{i}^{-}-\alpha_{j}^{+}}\left\langle\frac{D\left(\alpha_{i}^{-}\right)}{D\left(\alpha_{j}^{+}\right)}\right\rangle_{\triangle_{N+S}^{(2)}} \\
& W_{N}^{(2)}\left(\beta_{i}^{+}, \beta_{j}^{-}\right)=\frac{1}{\beta_{i}^{+}-\beta_{j}^{-}}\left\langle\frac{D\left(\beta_{j}^{-}\right)}{D\left(\beta_{i}^{+}\right)}\right\rangle_{\triangle_{N+S}^{(2)}} \\
& W_{N}^{(2)}\left(\beta_{i}^{+}, \beta_{j}^{+}\right)=\frac{C_{N+S+1}^{(2)}}{C_{N+S}^{(2)}}\left\langle\frac{1}{D\left(\beta_{i}^{+}\right) D\left(\beta_{j}^{+}\right)}\right\rangle_{\triangle_{N+S+1}^{(2)}}
\end{aligned}
$$

Proof. Since all the moments of $\mu$ are finite, and we are averaging functions of at most polynomial growth, it suffices to prove the theorem for the measures with compact support. Clearly, formula (4.1.1) remains intact under the operation of taking weak limits of measures $\mu$ having the same compact support. On the other hand, formula (4.1.1) was already proved in Theorem 2.7 .2 for all measures $\mu$ supported by a (large enough) finite set of points. Since any compactly supported probability measure can be weakly approximated by finitely supported ones, the proof is complete.

\subsection{Exact formulae for orthogonal $\beta=1$ and symplectic $\beta=4$ ensembles.}

Theorem 4.2.1. (Cases $\beta=1$ and $\beta=4$ ) (i) For any integers $N \geq 1$ and $S>1-N$, and finite sets of mutually distinct complex numbers

$$
\alpha=\left\{\alpha_{1}, \ldots, \alpha_{k}\right\}, \quad \beta=\left\{\beta_{1}, \ldots, \beta_{m}\right\}, \quad k-m=2 S,
$$

such that $\beta \cap \mathbb{R}=\varnothing$, one has

$$
\left\langle\frac{\prod_{i=1}^{k} D\left(\alpha_{i}\right)}{\prod_{i=1}^{m} D\left(\beta_{i}\right)}\right\rangle_{\triangle_{2 N}^{(1)}}=\frac{C_{2 N+2 S}^{(1)}}{C_{2 N}^{(1)}} \frac{\prod(\alpha ; \beta)}{V(\alpha) V(\beta)} \operatorname{Pf}\left[W_{N}^{(1)}(\alpha, \beta \mid \alpha, \beta)\right]
$$

where $W_{N}^{(1)}$ is a skew-symmetric $(k+m) \times(k+m)$ matrix with rows and columns parameterized by elements of $\alpha$ and $\beta$, and with matrix elements given by

$$
\begin{aligned}
& W_{N}^{(1)}\left(\alpha_{i}, \alpha_{j}\right)=\frac{C_{2 N+2 S-2}^{(1)}\left(\alpha_{i}-\alpha_{j}\right)\left\langle D\left(\alpha_{i}\right) D\left(\alpha_{j}\right)\right\rangle_{\triangle_{2 N+2 S-2}^{(1)}}}{C_{2 N+2 S}^{(1)}} \\
& W_{N}^{(1)}\left(\alpha_{i}, \beta_{j}\right)=\frac{1}{\alpha_{i}-\beta_{j}}\left\langle\frac{D\left(\alpha_{i}\right)}{D\left(\beta_{j}\right)}\right\rangle_{\triangle_{2 N+2 S}^{(1)}} \\
& W_{N}^{(1)}\left(\beta_{i}, \beta_{j}\right)=\frac{C_{2 N+2 S+2}^{(1)}}{C_{2 N+2 S}^{(1)}}\left(\beta_{i}-\beta_{j}\right)\left\langle\frac{1}{D\left(\beta_{i}\right) D\left(\beta_{j}\right)}\right\rangle_{\triangle_{2 N+2 S+2}^{(1)}}
\end{aligned}
$$

(ii) For any integers $N \geq 1$ and $S>1-N$, and finite sets of mutually distinct complex numbers 4.2.1) such that $\beta \cap \mathbb{R}=\varnothing$, one has

$$
\left\langle\frac{\prod_{i=1}^{k} D^{2}\left(\alpha_{i}\right)}{\prod_{i=1}^{m} D^{2}\left(\beta_{i}\right)}\right\rangle_{\triangle_{N}^{(4)}}=\frac{C_{N+S}^{(4)}}{C_{N}^{(4)}} \frac{\prod(\alpha ; \beta)}{V(\alpha) V(\beta)} \operatorname{Pf}\left[W_{N}^{(4)}(\alpha, \beta \mid \alpha, \beta)\right]
$$


where $W_{N}^{(4)}$ is a skew-symmetric matrix with rows and columns parameterized by elements of $\alpha$ and $\beta$, and with matrix elements given by

$$
\begin{aligned}
& W_{N}^{(4)}\left(\alpha_{i}, \alpha_{j}\right)=\frac{C_{N+S-1}^{(4)}}{C_{N+S}^{(4)}}\left(\alpha_{i}-\alpha_{j}\right)\left\langle D^{2}\left(\alpha_{i}\right) D^{2}\left(\alpha_{j}\right)\right\rangle_{\triangle_{N+S-1}^{(4)}} \\
& W_{N}^{(4)}\left(\alpha_{i}, \beta_{j}\right)=\frac{1}{\alpha_{i}-\beta_{j}}\left\langle\frac{D^{2}\left(\alpha_{i}\right)}{D^{2}\left(\beta_{j}\right)}\right\rangle_{\triangle_{N+S}^{(4)}} \\
& W_{N}^{(4)}\left(\beta_{i}, \beta_{j}\right)=\frac{C_{N+S+1}^{(4)}}{C_{N+S}^{(4)}}\left(\beta_{i}-\beta_{j}\right)\left\langle\frac{1}{D^{2}\left(\beta_{i}\right) D^{2}\left(\beta_{j}\right)}\right\rangle_{\triangle_{N+S+1}^{(4)}}
\end{aligned}
$$

Proof. Arguing as in the proof of Theorem 4.1.1 we see that it suffices to prove (4.2.2) and (4.2.3) for compactly supported measures $\mu$ with continuous density with respect to the Lebesgue measure:

$$
\mu(d x)=F(x) d x, \quad F \in C(\mathbb{R}), \quad \operatorname{supp}(F) \subset[-A, A], \quad A \in \mathbb{R}_{+} .
$$

Let us consider the case $\beta=1$ first. The idea is to reduce the statement to Theorem 3.8.3. Split the segment $[-A, A]$ into $M>2 N$ intervals of length $2 A / M$ :

$$
[-A, A]=I_{1} \cup \cdots \cup I_{M}, \quad I_{k}=\left[-A+\frac{2(k-1) A}{M},-A+\frac{2 k A}{M}\right],
$$

and take a discrete set $\mathfrak{X}$ with $2 M$ points such that each of the intervals $I_{k}$ contains exactly two points of $\mathfrak{X}$. One may take for example

$$
\mathfrak{X}=\left\{-A+\frac{A}{2 M},-A+\frac{3 A}{2 M} ;-A+\frac{5 A}{2 M},-A+\frac{7 A}{2 M} ; \ldots ; A-\frac{3 A}{2 M}, A-\frac{A}{2 M}\right\} .
$$

Then for any choice of $2 N$ intervals $I_{k_{j}}, j=1, \ldots, 2 N$, with $k_{1}<k_{2}<\cdots<k_{2 N}$, there exists a unique way to choose a point of $\mathfrak{X}$ in each of these intervals so that the chosen $2 N$ points of $\mathfrak{X}$ form a point configuration from $\operatorname{Con} f_{2 N}^{(1)}(\mathfrak{X})$. Conversely, any element of $\operatorname{Con} f_{2 N}^{(1)}(\mathfrak{X})$ produces $2 N$ intervals $I_{k_{j}}$ which contain the points of the configuration. Thus, for any continuous function $g:[-A, A]^{2 N} \rightarrow \mathbb{C}$,

$$
\left(\frac{M}{2 A}\right)^{2 N} \sum_{\left(x_{1}<\cdots<x_{2 N}\right) \in \operatorname{Conf} f_{2 N}^{(1)}(\mathfrak{X})} g\left(x_{1}, \ldots, x_{2 N}\right)
$$

can be viewed as a Riemannian sum for the integral

$$
\int_{-A<x_{1}<\cdots<x_{2 N}<A} g\left(x_{1}, \ldots, x_{2 N}\right) d x_{1} \cdots d x_{2 N} .
$$

Taking $M$ to $\infty$ brings the diameter of the partition $[-A, A]=I_{1} \cup \cdots \cup I_{M}$ to zero, and formula (4.2.2) directly follows from Theorem 3.8.3. The proof of (i) is complete.

Let consider the case $\beta=4$ now. We want to derive (4.2.3) from Theorem 3.8.2 Take $M>2 N$ and

$$
\mathfrak{X}=\left\{-A+\frac{(2 k-1) A}{M}\right\}_{k=1}^{M} \subset[-A, A] .
$$

Then for any continuous function $g:[-A, A]^{N} \rightarrow \mathbb{C}$,

$$
\begin{gathered}
\sum_{X=\left({ }^{l} x_{1}<x_{1}<\cdots<{ }^{l} x_{N}<x_{N}\right) \in C o n f_{2 N}^{(4)}(\mathfrak{X})} g\left(x_{1}, \ldots, x_{N}\right)|V(X)|=\left(\frac{M}{2 A}\right)^{N} \\
\times \sum_{\substack{x_{i} \in \mathfrak{X} \\
-A+\frac{A}{M}<x_{1}<\cdots<x_{N}}} g\left(x_{1}, \ldots, x_{N}\right) \prod_{1 \leq i<j \leq N}\left(x_{i}-x_{j}\right)^{2}\left(x_{i}-x_{j}-\frac{2 A}{M}\right)\left(x_{i}-x_{j}+\frac{2 A}{M}\right) \\
=\int_{-A<x_{1}<\cdots<x_{N}<A} g\left(x_{1}, \ldots, x_{N}\right) \prod_{1 \leq i<j \leq N}\left(x_{i}-x_{j}\right)^{4} d x_{1} \cdots d x_{N}+O\left(M^{-1}\right) .
\end{gathered}
$$


Thus, Theorem 3.8 .2 implies 4.2.3).

Remark 4.2.2. The conditions imposed on the measure $\mu$ at the beginning of this section can be relaxed. Namely, instead of finiteness of all the moments it suffices to require the convergence of the averages involved in (4.2.2) and (4.2.3), and instead of requiring all $C_{N}^{(\beta)}$ to be nonzero it also suffices to require that only of the constants involved in these formulas. This allows $\mu$ to have finite support.

Remark 4.2.3. The statements of Theorems 4.1.1 and 4.2.1 admit an interpretation in terms of random matrices. Indeed, the measures $\triangle_{N}^{(2)}, \triangle_{2 N}^{(1)}$, and $\triangle_{N}^{(4)}$ are radial parts (i.e., projections onto (different) eigenvalues) of the measures

$$
P(d H)=\text { const } \cdot \exp (-Q(H)) d H,
$$

where $Q(x)$ is an even degree polynomial with positive highest coefficient, and $H$ belongs to the linear space of $N \times N$ Hermitian, $2 N \times 2 N$ real symmetric, or $N \times N$ quaternion real Hermitian matrices, respectively. This corresponds to our measure $\mu$ being equal $\mu(d x)=\exp (-Q(x)) d x$ for $\beta=1$ and 2 , and $\mu(d x)=\exp (-2 Q(x)) d x$ for $\beta=4$. In the cases $\beta=1,2$ the characteristic polynomial of the random matrix $H$, $\operatorname{det}(\zeta-H)$, is exactly our product-function $D(\zeta)$, while for $\beta=4$ we have $\operatorname{det}(\zeta-H)=D^{2}(\zeta)$. Thus, in Theorems 1,2 we deal with average values of products and ratios of characteristic polynomials with respect to the measures on matrices introduced above. In the case $Q(x)=$ const $x^{2}$ these matrix probability spaces are called Gaussian Unitary $(\beta=2)$, Orthogonal $(\beta=1)$, and Symplectic $(\beta=4)$ Ensembles (GUE, GOE, GSE, for short).

Remark 4.2.4. Formulas (4.1.1), (4.2.2), 44.2.3) require that the total number of factors in the left-hand side is even (we count factors in both the numerator and the denominator). These formulas can be easily extended to the situation when the total number of factors is odd. In order to do that, one needs to take the even case and send one of the parameters $\alpha_{i}^{ \pm}, \beta_{j}^{ \pm}$in (4.1.1) and $\alpha_{i}, \beta_{j}$ in (4.2.2), (4.2.3) to $\infty$. Given that $D(\zeta) \sim \zeta^{N}$ in $\triangle_{N}^{(\beta)}$, the limiting formulas are readily obtained. After the limit transition, some of the two-point averages in the matrix elements of $W_{N}^{(\beta)}$ in the right-hand side turn into one-point averages of the form $\langle D(\gamma)\rangle,\langle 1 / D(\gamma)\rangle$ in the case $\beta=1,2$, or $\left\langle D^{2}(\gamma)\right\rangle,\left\langle 1 / D^{2}(\gamma)\right\rangle$ in the case $\beta=4$.

4.3. Correlation functions. Theorems 4.1.1 and 4.2.1 allow to compute the correlation functions of $\triangle_{N}^{(\beta)}$ for $\beta=1,2,4$ and reproduce fundamental results of the Random Matrix Theory.

Define the $n$th correlation measure of $\triangle_{N}^{(\beta)}$ by

$$
\rho_{n, N}^{(\beta)}\left(d x_{1}, \ldots, d x_{n}\right)=\frac{N !}{(N-n) !} \int_{x_{n+1}, x_{n+2}, \ldots, x_{N}} p_{N}^{(\beta)}\left(d x_{1}, \ldots, d x_{N}\right) .
$$

For a function $f(z)$ of a complex variable $\zeta$, which is continuous in both half-planes $\operatorname{Im} \zeta>0$ and $\operatorname{Im} \zeta<0$ up to the real axis, we will denote by $[f(\zeta)]_{\zeta=x}, x \in \mathbb{R}$, the difference of the limit values of $f(\zeta)$ as $\zeta \rightarrow x$ from bottom and from top divided by $2 \pi i$ :

$$
[f(\zeta)]_{x}=\frac{1}{2 \pi i}(f(x-i 0)-f(x+i 0)) .
$$

Theorem 4.3.1. Take any $n \geq 1$ and assume that near $n$ points $x_{1}, \ldots, x_{n} \in \mathbb{R}$ the measure $\mu$ is absolutely continuous with respect to the Lebesgue measure, and its density their is uniformly Hölder continuous. Then the nth correlation measure of $\triangle_{N}^{(\beta)}$ (or $\triangle_{2 N}^{(\beta)}$ for $\beta=1$ ) has a continuous density near $\left(x_{1}, \ldots, x_{n}\right)$ which is given by

- For $\beta=2$

$$
\rho_{n, N}^{(2)}\left(x_{1}, \ldots, x_{n}\right)=\operatorname{det}\left[K_{N}^{(2)}\left(x_{i}, x_{j}\right)\right]_{i, j=1}^{n}
$$

where for $x \neq y$ the kernel is given by

$$
K_{N}^{(2)}(x, y)=\frac{1}{x-y}\left[\left\langle\frac{D(x)}{D(\zeta)}\right\rangle_{\triangle_{N}^{(2)}}\right]_{\zeta=y},
$$


and for $x=y$ the kernel is defined by continuity.

- For $\beta=1$

$$
\rho_{n, 2 N}^{(1)}\left(x_{1}, \ldots, x_{n}\right)=\operatorname{Pf}\left[K_{2 N}^{(1)}\left(x_{i}, x_{j}\right)\right]_{i, j=1}^{n}
$$

where the skew-symmetric $2 \times 2$ matrix kernel for $x \neq y$ is given by

$$
K_{2 N}^{(1)}(x, y)=\left[\begin{array}{cc}
\frac{C_{2 N-2}^{(1)}}{C_{2 N}^{(1)}}(x-y)\langle D(x) D(y)\rangle_{\triangle_{2 N-2}^{(1)}} & \frac{1}{x-y}\left[\left\langle\frac{D(x)}{D(\zeta)}\right\rangle_{\triangle_{2 N}^{(1)}}\right]_{\zeta=y} \\
-\frac{1}{x-y}\left[\left\langle\frac{D(x)}{D(\zeta)}\right\rangle_{\triangle_{2 N}^{(1)}}\right]_{\zeta=y} & \frac{C_{2 N+2}^{(1)}}{C_{2 N}^{(1)}}(x-y)\left[\left\langle\frac{1}{D(\zeta) D(\eta)}\right\rangle_{\triangle_{2 N+2}^{(1)}}\right]_{\zeta=x, \eta=y}
\end{array}\right]
$$

and for $x=y$ the kernel is defined by continuity.

- For $\beta=4$

$$
\rho_{n, N}^{(4)}\left(x_{1}, \ldots, x_{n}\right)=\operatorname{Pf}\left[K_{N}^{(4)}\left(x_{i}, x_{j}\right)\right]_{i, j=1}^{n}
$$

where the skew-symmetric $2 \times 2$ matrix kernel for $x \neq y$ is given by

$$
K_{N}^{(4)}(x, y)=\left[\begin{array}{cc}
\frac{C_{N-1}^{(4)}}{2 C_{N}^{(4)}}(x-y)\left\langle D^{2}(x) D^{2}(y)\right\rangle_{\triangle_{N+1}^{(4)}} & \frac{1}{2(x-y)}\left[\left\langle\frac{D^{2}(x)}{D^{2}(\zeta)}\right\rangle_{\triangle_{N}^{(4)}}\right]_{\zeta=y} \\
-\frac{1}{2(x-y)}\left[\left\langle\frac{D^{2}(x)}{D^{2}(\zeta)}\right\rangle_{\triangle_{N}^{(4)}}\right]_{\zeta=y} & \frac{C_{N+1}^{(4)}}{2 C_{N}^{(4)}}(x-y)\left[\left\langle\frac{1}{D^{2}(\zeta) D^{2}(\eta)}\right\rangle_{\triangle_{N-1}^{(4)}}\right]_{\zeta=x, \eta=y}
\end{array}\right]
$$

and for $x=y$ the kernel is defined by continuity.

Proof. The Sokhotski-Plemelj formula implies that

$$
\begin{gathered}
\rho_{n, N}^{(\beta)}\left(x_{1}, \ldots, x_{n}\right)=\left[\left.\frac{\partial^{n}}{\partial u_{1} \cdots \partial u_{n}}\right|_{u=v}\left\langle\frac{D\left(u_{1}\right) \cdots D\left(u_{n}\right)}{D\left(v_{1}\right) \cdots D\left(v_{n}\right)}\right\rangle_{\triangle_{N}^{(\beta)}}\right]_{v_{1}=x_{1}, \ldots, v_{n}=x_{n}} \\
\rho_{n, N}^{(\beta)}\left(x_{1}, \ldots, x_{n}\right)=\frac{1}{2^{n}}\left[\left.\frac{\partial^{n}}{\partial u_{1} \cdots \partial u_{n}}\right|_{u=v}\left\langle\frac{D^{2}\left(u_{1}\right) \cdots D^{2}\left(u_{n}\right)}{D^{2}\left(v_{1}\right) \cdots D^{2}\left(v_{n}\right)}\right\rangle_{\triangle_{N}^{(\beta)}}\right]_{v_{1}=x_{1}, \ldots, v_{n}=x_{n}}
\end{gathered}
$$

We use the first formula for $\beta=1,2$ and the second one for $\beta=4$, and then proceed similarly to Propositions 2.8.3 and 3.9.1

Remark 4.3.2. If instead of asking for the measure $\mu$ to have nice density near $x_{1}, \ldots, x_{n}$, we require that $\mu$ is purely atomic near these points, then the formulas of Theorem 4.3.1 will continue to hold if we understand the symbol $[f(\zeta)]_{x}$ as the residue of the meromorphic function $f(\zeta)$ at the point $x$. The proof is very similar.

The one- and two-point averages

$$
\langle D(x)\rangle,\left\langle\frac{1}{D(x)}\right\rangle, \quad\langle D(x) D(y)\rangle,\left\langle\frac{D(x)}{D(y)}\right\rangle,\left\langle\frac{1}{D(x) D(y)}\right\rangle
$$

for $\beta=1,2$, and similar expressions with $D^{2}$ instead of $D$ for $\beta=4$, can all be evaluated in terms of (skew)-orthogonal polynomials associated with the measure $\mu$.

For $\beta=2$ one needs the usual orthogonal polynomials associated with $\mu$. The computation has been done by Strahov and Fyodorov [53], see also [5].

For $\beta=1$ and $\beta=4$ the skew-orthogonal polynomials associated to the skew-symmetric inner products

$$
\langle f, g\rangle_{1}=\frac{1}{2} \iint_{\mathbb{R}^{2}} \operatorname{sgn}(x-y) f(x) g(y) \mu(d x) \mu(d y),\langle f, g\rangle_{4}=\frac{1}{2} \int_{\mathbb{R}}\left(f(x) g^{\prime}(x)-f^{\prime}(x) g(x)\right) \mu(d x)
$$

need to be constructed. The expression of the one- and two-point functions in terms of these polynomials are exactly the same as in $\beta=2$ case modulo the change of the inner product. Since the proofs are essentially the same, we omit them here.

If one substitutes the expressions in terms of the (skew)-orthogonal polynomials into the formulas for the correlation kernels in Theorem 4.3.1 one recovers the classical formulas of Random Matrix Theory, see e.g. [56]. 


\section{Asymptotics}

This Section presents the asymptotic analysis of kernels and correlation functions of characteristic polynomials. The three cases are considered: the case of the Gaussian Unitary ensemble (GUE), the case of the Gaussian Orthogonal Ensemble (GOE), and the case of the Gaussian Symplectic Ensemble (GSE).

\subsection{Statement of Asymptotic Results.}

5.1.1. The Gaussian Unitary Ensemble. In the notation of Section 4, we consider the case of $\beta=2$, $\mu(d x)=e^{-x^{2}} d x$. The computations of correlation functions for GUE involve monic orthogonal polynomials defined by the following inner product:

$$
\langle f, g\rangle_{2}=\int_{-\infty}^{+\infty} d x e^{-x^{2}} f(x) g(x),
$$

and the relevant family of (monic) orthogonal polynomials $\left\{\pi_{n}\right\}, n=0,1,2, \ldots$, has the property

$$
\left\langle\pi_{l}, \pi_{k}\right\rangle_{2}=h_{k}^{(2)} \delta_{k l} .
$$

Remark 5.1.1. The inner products as well as the averages over ensembles are denoted by the same symbol \langle\rangle throughout the paper. These notations should not lead to a confusion.

In Section 4 we have found that averages of products and ratios of characteristic polynomials can be expressed in terms of kernels. In the case of GUE these kernels are

- $W_{N, I}^{(2)}(\zeta, \eta)=\frac{1}{h_{N}^{(2)}}(\zeta-\eta)\langle D(\zeta) D(\eta)\rangle_{\triangle_{N}^{(2)}} \quad \zeta \in \mathbb{C}, \eta \in \mathbb{C}$

- $W_{N, I I}^{(2)}(\zeta, \eta)=\frac{1}{\zeta-\eta}\left\langle\frac{D(\zeta)}{D(\eta)}\right\rangle_{\triangle_{N}^{(2)}}, \quad \zeta \in \mathbb{C}, \eta \in \mathbb{C} \backslash \mathbb{R}$

- $W_{N, I I I}^{(2)}(\zeta, \eta)=h_{N-1}^{(2)}(\zeta-\eta)\left\langle\frac{1}{D(\zeta) D(\eta)}\right\rangle_{\triangle_{N}^{(2)}} \zeta, \eta \in \mathbb{C} \backslash \mathbb{R}$

The following statement was proved in Ref. [53].

Theorem 5.1.2. (Scaling limits of kernels at the origin of the spectrum). The following limit relations hold:

$$
\begin{gathered}
\lim _{N \rightarrow \infty} \frac{1}{\sqrt{2 N}} W_{N, I}^{(2)}\left(\frac{\zeta}{\sqrt{2 N}}, \frac{\eta}{\sqrt{2 N}}\right)=\mathbb{S}_{G U E}^{I}(\zeta, \eta), \quad \lim _{N \rightarrow \infty} \frac{1}{\sqrt{2 N}} W_{N, I I}^{(2)}\left(\frac{\zeta}{\sqrt{2 N}}, \frac{\eta}{\sqrt{2 N}}\right)=\mathbb{S}_{G U E}^{I I}(\zeta, \eta) \\
\lim _{N \rightarrow \infty} \frac{1}{\sqrt{2 N}} W_{N, I I I}^{(2)}\left(\frac{\zeta}{\sqrt{2 N}}, \frac{\eta}{\sqrt{2 N}}\right)=\mathbb{S}_{G U E}^{I I I}(\zeta, \eta) .
\end{gathered}
$$

where

$$
\begin{aligned}
& \text { - } \mathbb{S}_{G U E}^{I}(\zeta, \eta)=\frac{1}{\pi}\left[\frac{\sin (\zeta-\eta)}{\zeta-\eta}\right], \quad \zeta \in \mathbb{C}, \eta \in \mathbb{C} . \\
& \text { - } \mathbb{S}_{G U E}^{I I}(\zeta, \eta)=\left\{\begin{array}{ll}
\frac{e^{i(\eta-\zeta)}}{\zeta-\eta} & \operatorname{Im} \eta>0, \\
\frac{e^{-i(\eta-\zeta)}}{\zeta-\eta} & \operatorname{Im} \eta<0 .
\end{array}, \quad \zeta \in \mathbb{C}, \quad \eta \in \mathbb{C} \backslash \mathbb{R} .\right. \\
& \text { - } \mathbb{S}_{G U E}^{I I I}(\zeta, \eta)=2 \pi i\left\{\begin{array}{cl}
\frac{e^{i(\zeta-\eta)}}{\zeta-\eta} & \operatorname{Im} \zeta>0, \operatorname{Im} \eta<0 \\
-\frac{e^{-i(\zeta-\eta)}}{\zeta-\eta} & \operatorname{Im} \zeta<0, \operatorname{Im} \eta>0 \\
0 & \text { otherwise. }
\end{array}\right.
\end{aligned}
$$


Theorem 5.1.3. For any integers $N \geq 1$ and $S>1-N$, and finite sets of mutually distinct complex numbers

$$
\begin{aligned}
\alpha^{-}=\left\{\alpha_{1}^{-}, \ldots, \alpha_{m_{1}}^{-}\right\}, & \alpha^{+}=\left\{\alpha_{1}^{+}, \ldots, \alpha_{k_{1}}^{-}\right\}, \\
\beta^{-}=\left\{\beta_{1}^{-}, \ldots, \beta_{m_{2}}^{-}\right\}, & \beta^{+}=\left\{\beta_{1}^{+}, \ldots, \beta_{k_{2}}^{+}\right\},
\end{aligned}
$$

with $\left|\alpha^{-}\right|-\left|\alpha^{+}\right|=\left|\beta^{-}\right|-\left|\beta^{+}\right|=S$ and

$$
\alpha^{-} \cap \alpha^{+}=\varnothing, \quad \alpha^{+} \cap \mathbb{R}=\beta^{+} \cap \mathbb{R}=\varnothing, \quad \beta^{-} \cap \beta^{+}=\varnothing,
$$

one has

$$
\begin{aligned}
& \lim _{N \rightarrow \infty}\left\{\frac{1}{\mathrm{~T}^{(2)}(N)}\left\langle\frac{\prod_{i=1}^{m_{1}} D\left(\frac{\alpha_{i}^{-}}{\sqrt{2 N}}\right) \prod_{i=1}^{m_{2}} D\left(\frac{\beta_{i}^{-}}{\sqrt{2 N}}\right)}{\prod_{j=1}^{k_{1}} D\left(\frac{\alpha_{i}^{+}}{\sqrt{2 N}}\right) \prod_{j=1}^{k_{2}} D\left(\frac{\beta_{j}^{+}}{\sqrt{2 N}}\right)}\right\rangle_{\triangle_{N}^{(2)}}\right\} \\
& =(-1)^{\frac{\left(\left|\beta^{-}\right|+\left|\alpha^{-}\right|\right)^{2}+\left(\left|\beta^{-}\right|-\left|\alpha^{-}\right|\right)}{2}} \frac{\prod\left(\alpha^{-} ; \alpha^{+}\right) \prod\left(\beta^{-} ; \beta^{+}\right)}{V\left(\alpha^{-}\right) V\left(\alpha^{+}\right) V\left(\beta^{-}\right) V\left(\beta^{+}\right)} \operatorname{det}\left[\mathbb{S}_{G U E}\left(\alpha^{-}, \beta^{+} \mid \beta^{-}, \alpha^{+}\right)\right],
\end{aligned}
$$

where

$$
\frac{1}{\mathrm{~T}^{(2)}(N)}=\frac{C_{N}^{(2)}}{C_{N+S}^{(2)}}(2 N)^{\frac{S^{2}}{2}},
$$

$\mathbb{S}_{G U E}\left(\alpha^{-}, \beta^{+} \mid \beta^{-}, \alpha^{+}\right)$is a matrix with rows parameterized by elements of $\alpha^{-}$and $\beta^{+}$, columns parameterized by $\beta^{-}, \alpha^{+}$, and with matrix elements:

- $\mathbb{S}_{G U E}\left(\alpha_{i}^{-}, \beta_{j}^{-}\right)=\mathbb{S}_{G U E}^{I}\left(\alpha_{i}^{-}, \beta_{j}^{-}\right)$

- $\mathbb{S}_{G U E}\left(\alpha_{i}^{-}, \alpha_{j}^{+}\right)=\mathbb{S}_{G U E}^{I I}\left(\alpha_{i}^{-}, \alpha_{j}^{+}\right)$

- $\mathbb{S}_{G U E}\left(\beta_{i}^{+}, \beta_{j}^{-}\right)=\mathbb{S}_{G U E}^{I I}\left(\beta_{j}^{-}, \beta_{i}^{+}\right)$

- $\mathbb{S}_{G U E}\left(\beta_{i}^{+}, \alpha_{j}^{+}\right)=\mathbb{S}_{G U E}^{I I I}\left(\beta_{i}^{+}, \alpha_{j}^{+}\right)$

The constant $C_{N}^{(2)}$ can be explicitly computed, see Mehta [44]:

$$
C_{N}^{(2)}=\pi^{\frac{N}{2}} 2^{-\frac{N(N-1)}{2}} \cdot \prod_{j=1}^{N} j !
$$

5.1.2. The Gaussian Orthogonal Ensemble. Let us now consider the case $\beta=1, \mu(d x)=e^{-\frac{x^{2}}{2}} d x$. The computations of correlation functions for GOE involve skew orthogonal polynomials. They are defined by the following skew symmetric inner product:

$$
\langle f, g\rangle_{1}=\frac{1}{2} \int_{-\infty}^{+\infty} d x e^{-\frac{x^{2}}{2}} \int_{-\infty}^{+\infty} d y e^{-\frac{y^{2}}{2}} \operatorname{sgn}(y-x) f(x) g(y) .
$$

Specifically, the relevant family of (monic) skew orthogonal polynomials $\left\{p_{n}\right\}, n=0,1,2, \ldots$, is given by

$$
\left\langle p_{2 k}, p_{2 l+1}\right\rangle_{1}=-\left\langle p_{2 l+1}, p_{2 k}\right\rangle_{1}=h_{k}^{(1)} \delta_{k l} .
$$

and by the condition that all other skew symmetric products between these polynomials are zeros.

The averages of products and ratios of characteristic polynomials can be expressed in terms of kernels. In the case of GOE these kernels are

$$
\begin{aligned}
& \text { - } W_{N, I}^{(1)}(\zeta, \eta)=\frac{1}{h_{N}^{(1)}}(\zeta-\eta)\langle d(\zeta) d(\eta)\rangle_{\triangle_{2 N}^{(1)}} \quad \zeta \in \mathbb{C}, \eta \in \mathbb{C} . \\
& \text { - } W_{N, I I}^{(1)}(\zeta, \eta)=\frac{1}{\zeta-\eta}\left\langle\frac{d(\zeta)}{d(\eta)}\right\rangle_{\triangle_{2 N}^{(1)}}, \quad \zeta \in \mathbb{C}, \eta \in \mathbb{C} \backslash \mathbb{R} . \\
& \text { - } W_{N, I I I}^{(1)}(\zeta, \eta)=h_{N-1}^{(1)}(\zeta-\eta)\left\langle\frac{1}{d(\zeta) d(\eta)}\right\rangle_{\triangle_{2 N}^{(1)}} \quad \zeta, \eta \in \mathbb{C} \backslash \mathbb{R} .
\end{aligned}
$$


The kernels $W_{N, I}^{(1)}(\zeta, \eta), W_{N, I I}^{(1)}(\zeta, \eta)$, and $W_{N, I I I}^{(1)}(\zeta, \eta)$ admit representations as Christoffel-Darboux type sums. Namely,

$$
\begin{gathered}
W_{N, I}^{(1)}(\zeta, \eta)=\sum_{i=0}^{N} \frac{p_{2 i+1}(\zeta) p_{2 i}(\eta)-p_{2 i}(\zeta) p_{2 i+1}(\eta)}{h_{i}^{(1)}} \\
W_{N, I I}^{(1)}(\zeta, \eta)=\frac{1}{\zeta-\eta}+\sum_{i=0}^{N-1} \frac{p_{2 i+1}(\zeta) h_{2 i}(\eta)-p_{2 i}(\zeta) h_{2 i+1}(\eta)}{h_{i}^{(1)}} \\
W_{N, I I I}^{(1)}(\zeta, \eta)=\sum_{i=0}^{N-2} \frac{h_{2 i+1}(\zeta) h_{2 i}(\eta)-h_{2 i}(\zeta) h_{2 i+1}(\eta)}{h_{i}^{(1)}}+\left\langle R_{\zeta}, R_{\eta}\right\rangle_{1} .
\end{gathered}
$$

Here $R_{\zeta} \equiv(\zeta-x)^{-1}$, and the functions $h_{k}$ are the skew symmetric products between skew orthogonal polynomials and $R_{\zeta}$ :

$$
h_{k}(\eta)=\left\langle p_{k}, R_{\eta}\right\rangle_{1}, \quad \eta \in \mathbb{C} \backslash \mathbb{R} .
$$

Theorem 5.1.4. (Scaling limits of kernels at the origin of the spectrum). The following limit relations hold:

$$
\begin{gathered}
\lim _{N \rightarrow \infty} \frac{1}{2 N} W_{N, I}^{(1)}\left(\frac{\zeta}{\sqrt{2 N}}, \frac{\eta}{\sqrt{2 N}}\right)=\mathbb{S}_{G O E}^{I}(\zeta, \eta), \quad \lim _{N \rightarrow \infty} \frac{1}{\sqrt{2 N}} W_{N, I I}^{(1)}\left(\frac{\zeta}{\sqrt{2 N}}, \frac{\eta}{\sqrt{2 N}}\right)=\mathbb{S}_{G O E}^{I I}(\zeta, \eta), \\
\lim _{N \rightarrow \infty} W_{N, I I I}^{(1)}\left(\frac{\zeta}{\sqrt{2 N}}, \frac{\eta}{\sqrt{2 N}}\right)=\mathbb{S}_{G O E}^{I I I}(\zeta, \eta) .
\end{gathered}
$$

where

$$
\begin{aligned}
& \text { - } \mathbb{S}_{G O E}^{I}(\zeta, \eta)=-\frac{1}{\pi} \frac{d}{d \zeta}\left[\frac{\sin (\zeta-\eta)}{\zeta-\eta}\right], \quad \zeta \in \mathbb{C}, \eta \in \mathbb{C} . \\
& \text { - } \mathbb{S}_{G O E}^{I I}(\zeta, \eta)= \begin{cases}\frac{e^{i(\eta-\zeta)}}{\zeta-\eta} & \operatorname{Im} \eta>0, \\
\frac{e^{-i(\eta-\zeta)}}{\zeta-\eta} & \operatorname{Im} \eta<0 .\end{cases} \\
& \text { - } \mathbb{S}_{G O E}^{I I I}(\zeta, \eta)=2 \pi i\left\{\begin{array}{cl}
\int_{1}^{+\infty} \frac{e^{i(\zeta-\eta) t} d t}{t} & \operatorname{Im} \zeta>0, \operatorname{Im} \eta<0 \\
-\int_{1}^{+\infty} \frac{e^{-i(\zeta-\eta) t} d t}{t} & \operatorname{Im} \zeta<0, \operatorname{Im} \eta>0 \\
0 & \text { otherwise. }
\end{array}\right.
\end{aligned}
$$

Theorem 5.1.5. For any integers $N \geq 1$ and $S>1-N$, and finite sets of mutually distinct complex numbers

such that

$$
\alpha=\left\{\alpha_{1}, \ldots, \alpha_{k}\right\}, \quad \beta=\left\{\beta_{1}, \ldots, \beta_{m}\right\}, \quad k-m=2 S
$$

one has

$$
\beta \cap \mathbb{R}=\varnothing
$$

$$
\lim _{N \rightarrow \infty}\left\{\frac{1}{\mathrm{~T}^{(1)}(N)}\left\langle\frac{\prod_{i=1}^{k} D\left(\frac{\alpha_{i}}{\sqrt{2 N}}\right)}{\prod_{i=1}^{m} D\left(\frac{\beta_{i}}{\sqrt{2 N}}\right)}\right\rangle_{\triangle_{2 N}^{(1)}}\right\}=\frac{\prod(\alpha ; \beta)}{V(\alpha) V(\beta)} \operatorname{Pf}\left[\mathbb{S}_{G O E}(\alpha, \beta \mid \alpha, \beta)\right]
$$

where

$$
\frac{1}{\mathrm{~T}^{(1)}(N)}=\frac{C_{2 N}^{(1)}}{C_{2 N+2 S}^{(1)}}(2 N)^{\frac{k m}{2}-\frac{k(k+1)}{4}-\frac{m(m-1)}{4}}
$$

$\mathbb{S}_{G O E}(\alpha, \beta \mid \alpha, \beta)$ is a skew symmetric $(k+m) \times(k+m)$ matrix with rows and columns parameterized by elements of $\alpha$ and $\beta$, and with matrix elements: 
- $\mathbb{S}_{G O E}\left(\alpha_{i}, \alpha_{j}\right)=\mathbb{S}_{G O E}^{I}\left(\alpha_{i}, \alpha_{j}\right)$

- $\mathbb{S}_{G O E}\left(\alpha_{i}, \beta_{j}\right)=\mathbb{S}_{G O E}^{I I}\left(\alpha_{i}, \beta_{j}\right)$

- $\mathbb{S}_{G O E}\left(\beta_{i}, \beta_{j}\right)=\mathbb{S}_{G O E}^{I I I}\left(\beta_{i}, \beta_{j}\right)$

The constants $C_{2 N}^{(1)}$ can be explicitly computed, see Mehta [44]:

$$
(2 N) ! C_{2 N}^{(1)}=(2 \pi)^{N} \prod_{j=0}^{2 N-1} \frac{\Gamma\left(\frac{3}{2}+\frac{j}{2}\right)}{\Gamma\left(\frac{3}{2}\right)}
$$

5.1.3. The Gaussian Symplectic Ensemble. Finally, let us take $\beta=4, \mu(d x)=e^{-x^{2}} d x$. Given two functions $f, g$ the skew symmetric inner product for the Gaussian Symplectic Ensemble (GSE) is defined by

$$
\langle f, g\rangle_{4}=\frac{1}{2} \int_{-\infty}^{+\infty} d x e^{-x^{2}}\left(f(x) g^{\prime}(x)-f^{\prime}(x) g(x)\right) .
$$

The family of monic skew orthogonal polynomials associated with GSE is introduced by

$$
\left\langle p_{2 k}, p_{2 l+1}\right\rangle_{4}=-\left\langle p_{2 l+1}, p_{2 k}\right\rangle_{4}=h_{k}^{(4)} \delta_{k l} .
$$

All other brackets are zeros. In the case of GSE the kernels that determine averages of characteristic polynomials are:

$$
\begin{aligned}
& \text { - } W_{N, I}^{(4)}(\zeta, \eta)=\frac{1}{h_{N}^{(4)}}(\zeta-\eta)\left\langle D^{2}(\zeta) D^{2}(\eta)\right\rangle_{\triangle_{N}^{(4)}}, \zeta \in \mathbb{C}, \eta \in \mathbb{C} . \\
& \text { - } W_{N, I I}^{(4)}(\zeta, \eta)=\frac{1}{\zeta-\eta}\left\langle\frac{D^{2}(\zeta)}{D^{2}(\eta)}\right\rangle_{\triangle_{N}^{(4)}}, \zeta \in \mathbb{C}, \eta \in \mathbb{C} \backslash \mathbb{R} . \\
& \text { - } W_{N, I I I}^{(4)}(\zeta, \eta)=h_{N-1}^{(4)}(\zeta-\eta)\left\langle\frac{1}{D^{2}(\zeta) D^{2}(\eta)}\right\rangle_{\triangle_{N}^{(4)}}, \zeta \in \mathbb{C} \backslash \mathbb{R}, \eta \in \mathbb{C} \backslash \mathbb{R} .
\end{aligned}
$$

Similar to the case of $G O E$ the following expressions were obtained:

$$
\begin{gathered}
W_{N, I}^{(4)}(\zeta, \eta)=\frac{1}{h_{N}^{(4)}} \sum_{i=0}^{N} \frac{p_{2 i+1}(\zeta) p_{2 i}(\eta)-p_{2 i}(\zeta) p_{2 i+1}(\eta)}{h_{i}^{(4)}} \\
W_{N, I I}^{(4)}(\zeta, \eta)=\frac{1}{\zeta-\eta}+\sum_{i=0}^{N-1} \frac{p_{2 i+1}(\zeta) h_{2 i}(\eta)-p_{2 i}(\zeta) h_{2 i+1}(\eta)}{h_{i}^{(4)}} \\
W_{N, I I I}^{(4)}(\zeta, \eta)=h_{N-1}^{(4)}\left[\sum_{i=0}^{N-2} \frac{h_{2 i+1}(\zeta) h_{2 i}(\eta)-h_{2 i}(\zeta) h_{2 i+1}(\eta)}{h_{i}^{(4)}}+\left\langle R_{\zeta}, R_{\eta}\right\rangle_{4}\right]
\end{gathered}
$$

Theorem 5.1.6. (Scaling limits of kernels at the origin of the spectrum). The following limit relations hold

$$
\begin{gathered}
\lim _{N \rightarrow \infty} W_{N, I}^{(4)}\left(\frac{\zeta}{\sqrt{2 N}}, \frac{\eta}{\sqrt{2 N}}\right)=\mathbb{S}_{G S E}^{I}(\zeta, \eta), \quad \lim _{N \rightarrow \infty} \frac{1}{\sqrt{2 N}} W_{N, I I}^{(4)}\left(\frac{\zeta}{\sqrt{2 N}}, \frac{\eta}{\sqrt{2 N}}\right)=\mathbb{S}_{G S E}^{I I}(\zeta, \eta), \\
\lim _{N \rightarrow \infty} \frac{1}{4 N} W_{N, I I I}^{(4)}\left(\frac{\zeta}{2 \sqrt{N}}, \frac{\eta}{2 \sqrt{N}}\right)=\mathbb{S}_{G S E}^{I I I}(\zeta, \eta) .
\end{gathered}
$$

where

- $\mathbb{S}_{G S E}^{I}(\zeta, \eta)=\frac{1}{\pi} \int_{0}^{1} d x \frac{\sin (\zeta-\eta) x}{x}, \quad \zeta \in \mathbb{C}, \quad \eta \in \mathbb{C}$.

- $\mathbb{S}_{G S E}^{I I}(\zeta, \eta)=\left\{\begin{array}{ll}\frac{e^{i(\eta-\zeta)}}{\zeta-\eta} & \operatorname{Im} \eta>0, \\ \frac{e^{-i(\eta-\zeta)}}{\zeta-\eta} & \operatorname{Im} \eta<0 .\end{array}, \zeta \in \mathbb{C}, \quad \eta \in \mathbb{C} \backslash \mathbb{R}\right.$. 


$$
\text { - } \mathbb{S}_{G S E}^{I I I}(\zeta, \eta)=2 \pi i\left\{\begin{array}{ll}
-\frac{d}{d \zeta}\left[\frac{e^{-i(\zeta-\eta)}}{\zeta-\eta}\right] & \operatorname{Im} \zeta<0, \operatorname{Im} \eta>0 \\
\frac{d}{d \zeta}\left[\frac{e^{i(\zeta-\eta)}}{\zeta-\eta}\right] & \operatorname{Im} \zeta>0, \operatorname{Im} \eta<0 \\
0 & \text { otherwise. }
\end{array}, \zeta, \eta \in \mathbb{C} \backslash \mathbb{R} .\right.
$$

Theorem 5.1.7. For any integers $N \geq 1$ and $S>1-N$, and finite sets of mutually distinct complex numbers

$$
\alpha=\left\{\alpha_{1}, \ldots, \alpha_{k}\right\}, \quad \beta=\left\{\beta_{1}, \ldots, \beta_{m}\right\}, \quad k-m=2 S
$$

such that

$$
\beta \cap \mathbb{R}=\varnothing
$$

one has

$$
\lim _{N \rightarrow \infty}\left\{\frac{1}{\mathrm{~T}^{(4)}(N)}\left\langle\frac{\prod_{i=1}^{k} d\left(\frac{\alpha_{i}}{\sqrt{2 N}}\right)}{\prod_{i=1}^{m} d\left(\frac{\beta_{i}}{\sqrt{2 N}}\right)}\right\rangle_{\triangle_{N}^{(4)}\left(e^{-x^{2} / 2}\right)}\right\}=\frac{\prod(\alpha ; \beta)}{V(\alpha) V(\beta)} \operatorname{Pf}\left[\mathbb{S}_{G S E}(\alpha, \beta \mid \alpha, \beta)\right]
$$

where

$$
\frac{1}{\mathrm{~T}^{(4)}(N)}=\frac{C_{N}^{(4)}}{C_{N+S}^{(4)}}(2 N)^{\frac{k m}{2}-\frac{k(k-1)}{4}-\frac{m(m+1)}{4}}
$$

$\mathbb{S}_{G S E}(\alpha, \beta \mid \alpha, \beta)$ is a skew symmetric $(k+m) \times(k+m)$ matrix with rows and columns parameterized by elements of $\alpha$ and $\beta$, and with matrix elements:

- $\mathbb{S}_{G S E}\left(\alpha_{i}, \alpha_{j}\right)=\mathbb{S}_{G S E}^{I}\left(\alpha_{i}, \alpha_{j}\right)$

- $\mathbb{S}_{G S E}\left(\alpha_{i}, \beta_{j}\right)=\mathbb{S}_{G S E}^{I I}\left(\alpha_{i}, \beta_{j}\right)$

- $\mathbb{S}_{G S E}\left(\beta_{i}, \beta_{j}\right)=\mathbb{S}_{G S E}^{I I I}\left(\beta_{i}, \beta_{j}\right)$

The constants $C_{N}^{(4)}$ can be explicitly computed, see Mehta [44]:

$$
N ! C_{N}^{(4)}=\frac{(2 \pi)^{N(N+1 / 2)}}{2^{N(N+1 / 2)}}\left[\prod_{j=0}^{N-1}(2 j+2) !\right]
$$

The following Sections include the proofs of Theorems 5.1.4 and 5.1.6

Remark 5.1.8. As we have seen in Sections 3.9, 4.3 the correlation functions of GUE, GOE, GSE can be expressed through 2-point averages of characteristic polynomials, see Theorem 4.3.1, If we insert the asymptotic expressions for $\mathbb{S}_{G U E}, \mathbb{S}_{G O E}, \mathbb{S}_{G S E}$ to the formulas of Theorem 4.3.1 we obtain the following asymptotic formulas for the correlation kernels:

$$
\begin{gathered}
\lim _{N \rightarrow \infty} \frac{1}{\sqrt{2 N}} K_{N}^{(2)}\left(\frac{x}{\sqrt{2 N}}, \frac{y}{\sqrt{2 N}}\right)=\frac{\sin (x-y)}{\pi(x-y)} \\
\lim _{N \rightarrow \infty}\left(\begin{array}{cc}
\frac{1}{\sqrt{2 N}} & 0 \\
0 & 1
\end{array}\right) K_{N}^{(1)}\left(\frac{x}{\sqrt{2 N}}, \frac{y}{\sqrt{2 N}}\right)\left(\begin{array}{cc}
\frac{1}{\sqrt{2 N}} & 0 \\
0 & 1
\end{array}\right)=\frac{1}{\pi}\left(\begin{array}{cc}
-\frac{d}{d x} \frac{\sin (x-y)}{x-y} & \frac{\sin (x-y)}{x-y} \\
-\frac{\sin (x-y)}{x-y} & -\int_{1}^{+\infty} \frac{d t \sin (x-y) t}{t}
\end{array}\right) \\
\lim _{N \rightarrow \infty}\left(\begin{array}{cc}
\frac{1}{\sqrt{2 N}} & 0 \\
0 & 1
\end{array}\right) K_{N}^{(4)}\left(\frac{x}{\sqrt{2 N}}, \frac{y}{\sqrt{2 N}}\right)\left(\begin{array}{cc}
\frac{1}{\sqrt{2 N}} & 0 \\
0 & 1
\end{array}\right)=\frac{1}{2 \pi}\left(\begin{array}{cc}
\int_{0}^{1} \frac{d t \sin (x-y) t}{t} & \frac{\sin (x-y)}{x-y} \\
-\frac{\sin (x-y)}{x-y} & -\frac{d}{d x} \frac{\sin (x-y)}{x-y}
\end{array}\right)
\end{gathered}
$$


The expression in the righthand sides are well known ${ }^{3}$, see e. g. Forrester [27, Chapter 5. To derive these expressions we observe that

$$
\begin{gathered}
{\left[\mathbb{S}_{G U E}^{I I}(x, \eta)\right]_{\eta=y}=\left[\mathbb{S}_{G O E}^{I I}(x, \eta)\right]_{\eta=y}=\left[\mathbb{S}_{G S E}^{I I}(x, \eta)\right]_{\eta=y}=\frac{\sin (x-y)}{\pi(x-y)}} \\
{\left[\mathbb{S}_{G O E}^{I I I}(\zeta, \eta)\right]_{\zeta=x, \eta=y}=-\frac{1}{\pi} \int_{1}^{+\infty} \frac{d t \sin (x-y) t}{t}} \\
{\left[\mathbb{S}_{G S E}^{I I I}(\zeta, \eta)\right]_{\zeta=x, \eta=y}=-\frac{1}{\pi} \frac{d}{d x} \frac{\sin (x-y)}{x-y}}
\end{gathered}
$$

\subsection{Summation formulae for kernels.}

\subsubsection{Summation formulae for kernels in the GOE case.}

Proposition 5.2.1. The Christoffel-Darboux type sum constructed from the skew orthogonal polynomials $\left\{p_{n}\right\}, n=0,1,2, \ldots$, is representable in terms of the orthonormal functions associated with the Hermite polynomials:

$$
\begin{aligned}
& \sum_{i=0}^{N-1} \frac{p_{2 i}(x) p_{2 i+1}(y)-p_{2 i}(x) p_{2 i+1}(y)}{h_{i}}= \\
& \quad e^{\frac{x^{2}+y^{2}}{2}} \frac{c_{N}}{c_{N-1}}\left[\frac{d}{d x}\left(\frac{\psi_{N}(x) \psi_{N-1}(y)-\psi_{N-1}(x) \psi_{N}(y)}{x-y}\right)+\psi_{N-1}(y) \psi_{N}(x)\right]
\end{aligned}
$$

where the functions $\psi_{n}(x)$ are orthonormal functions associated with the Hermite polynomials. Namely,

$$
\psi_{n}(x)=\frac{\pi_{n}(x) e^{-\frac{x^{2}}{2}}}{c_{n}}=\frac{H_{n}(x) e^{-\frac{x^{2}}{2}}}{2^{n} c_{n}}, \quad \int \psi_{n}(x) \psi_{m}(x) d x=\delta_{n m} .
$$

Here $H_{n}(x)$ are the Hermite polynomials, $\pi_{n}$ are the monic orthogonal polynomials defined by the weight $w(x)=e^{-x^{2}}$, and the coefficients $c_{n}$ are their norms,

$$
c_{n}=\pi^{1 / 4} 2^{-n / 2} \sqrt{n !}
$$

This result is well-known and can be found in the literature on Random Matrix Theory (see, for example, Forrester, Honner and Nagao [29], Adler, Forrester, Nagao, and van Moerbeke [1, Widom [58]).

Proposition 5.2.1 lead us to the representations for kernels which are suitable for the asymptotic analysis.

Proposition 5.2.2. The kernels $W_{N, I}^{(1)}(\zeta, \eta), W_{N, I I}^{(1)}(\zeta, \eta)$, and $W_{N, I I I}^{(1)}(\zeta, \eta)$ are representable in terms of the orthonomal functions $\psi_{n}(x)$ defined by equation (5.2.2), and by their Cauchy type transforms $\Psi_{n}(\zeta)$,

$$
\Psi_{n}(\zeta)=\int \frac{d x e^{-x^{2} / 2} \psi_{n}(x)}{x-\zeta} ; \quad \zeta \in \mathbb{C} \backslash \mathbb{R}
$$

Namely,

$$
W_{N, I}^{(1)}(\zeta, \eta)=-\frac{c_{N+1}}{c_{N}} e^{\frac{\zeta^{2}+\eta^{2}}{2}}\left[\frac{d}{d \zeta}\left(\frac{\psi_{N+1}(\zeta) \psi_{N}(\eta)-\psi_{N}(\zeta) \psi_{N+1}(\eta)}{\zeta-\eta}\right)+\psi_{N}(\eta) \psi_{N+1}(\zeta)\right]
$$

\footnotetext{
${ }^{3}$ The correlation functions in $\beta=1,4$ cases are usually written as quaternion determinants while we use pfaffian representations. The relation between them is explained in Section 3.9]
} 


$$
\begin{gathered}
W_{N, I I}^{(1)}(\zeta, \eta)=\frac{c_{N}}{c_{N-1}} e^{\zeta^{2} / 2}\left[\frac{\Psi_{N}(\eta) \psi_{N-1}(\zeta)-\Psi_{N-1}(\eta) \psi_{N}(\zeta)}{\zeta-\eta}\right. \\
\left.+\frac{1}{2} \psi_{N-1}(\zeta) \iint \frac{d x d y \psi_{N}(x) e^{-y^{2} / 2} \operatorname{sgn}(y-x)}{\eta-y}\right] \\
W_{N, I I I}^{(1)}(\zeta, \eta)=\frac{1}{2} \frac{c_{N-1}}{c_{N-2}}\left[\Psi_{N-1}(\zeta) \iint \frac{d x d y e^{-y^{2} / 2} \operatorname{sgn}(y-x) \psi_{N-2}(x)}{(y-\eta)(x-\zeta)}\right. \\
-\Psi_{N-2}(\zeta) \iint \frac{d x d y e^{-y^{2} / 2} \operatorname{sgn}(y-x) \psi_{N-1}(x)}{(y-\eta)(x-\zeta)} \\
\left.-1 / 2 \iint \frac{d x d y e^{-y^{2} / 2} \operatorname{sgn}(y-x) \psi_{N-1}(x)}{(y-\zeta)} \iint \frac{d x d y e^{-y^{2} / 2} \operatorname{sgn}(y-x) \psi_{N-2}(x)}{(y-\eta)}\right] .
\end{gathered}
$$

Proof. Equation (5.2.5) is obtained immediately from equations (5.2.1) and (5.1.1). Let us derive formula (5.2.6). We start from the representation of the second kernel as the Christoffel-Darboux type sum, equation (5.1.2). In this representation we rewrite the functions $h_{2 i}(\eta), h_{2 i+1}(\eta)$ explicitly as integral transformations (equation (5.1.4) ) of the monic skew orthogonal polynomials $p_{2 i}$ and $p_{2 i+1}$. Then we apply formula (5.2.1) and obtain

$$
\begin{aligned}
& W_{N, I I}^{(1)}(\zeta, \eta)=\frac{1}{\zeta-\eta}+\frac{1}{2} e^{\zeta^{2} / 2} \frac{c_{N}}{c_{N-1}}[ \\
& \quad \iint \frac{d x d y e^{-y^{2} / 2} \operatorname{sgn}(y-x)}{\eta-y} \frac{d}{d x}\left(\frac{\psi_{N}(x) \psi_{N-1}(\zeta)-\psi_{N-1}(x) \psi_{N}(\zeta)}{x-\zeta}\right) \\
& \left.\quad+\psi_{N-1}(\zeta) \iint \frac{d x d y \psi_{N}(x) e^{-y^{2} / 2} \operatorname{sgn}(y-x)}{\eta-y}\right]
\end{aligned}
$$

The first term in the brackets is simplified if we perform the integration by parts. After that we use

$$
\frac{1}{(y-\zeta)(y-\eta)}=\frac{1}{\zeta-\eta}\left(\frac{1}{y-\zeta}-\frac{1}{y-\eta}\right)
$$

and decompose the resulting integral into two terms. In each term the integrals are the Cauchy type transforms $\Psi_{n}$ of functions $\psi_{n}$. Now we apply the well-known formula

$$
\Psi_{N}(\zeta) \psi_{N-1}(\zeta)-\Psi_{N-1}(\zeta) \psi_{N}(\zeta)=\frac{c_{N-1}}{c_{N}} e^{-\zeta^{2} / 2}
$$

This formula follows, for example, from the fact that the determinant of the solution of the Riemann-Hilbert problem for orthogonal polynomials is identically 1, see Deift [22], page 44. Then one term (the first term in equation (5.2.8) ) is cancelled with $\frac{1}{\zeta-\eta}$ and equation (5.2.6) is obtained. Equation (5.2.7) is derived from equations (5.1.3) and (5.2.1) by the same procedure.

It is convenient to introduce two functions,

$$
I_{N}(\eta)=-\iint \frac{d x d y e^{-y^{2} / 2} \operatorname{sgn}(y-x) \psi_{N-1}(x)}{y-\eta},
$$

and

$$
E_{N}(\zeta, \eta)=-\iint \frac{d x d y e^{-y^{2} / 2} \operatorname{sgn}(y-x) \psi_{N-1}(x)}{(y-\eta)(x-\zeta)}
$$

Then the kernels $W_{N, I I}^{(1)}(\zeta, \eta)$ and $W_{N, I I I}^{(1)}(\zeta, \eta)$ can be rewritten as follows,

$$
W_{N, I I}^{(1)}(\zeta, \eta)=\frac{c_{N}}{c_{N-1}} e^{\zeta^{2} / 2}\left[\frac{\Psi_{N}(\eta) \psi_{N-1}(\zeta)-\Psi_{N-1}(\eta) \psi_{N}(\zeta)}{\zeta-\eta}+\frac{1}{2} \psi_{N-1}(\zeta) I_{N}(\eta)\right],
$$


and

$$
W_{N, I I I}^{(1)}(\zeta, \eta)=\frac{1}{2} \frac{c_{N-1}}{c_{N-2}}\left[E_{N-1}(\zeta, \eta) \Psi_{N-2}(\zeta)-E_{N-2}(\zeta, \eta) \Psi_{N-1}(\zeta)-\frac{1}{2} I_{N-1}(\zeta) I_{N-2}(\eta)\right] .
$$

5.2.2. Summation formulae for kernels in the GSE case. Given two functions, $f(x)$ and $g(x)$, introduce new skew symmetric brackets $($,$) by$

$$
(f, g)=\frac{1}{2} \int d x\left(f(x) g^{\prime}(x)-f^{\prime}(x) g(x)\right)
$$

It is easy to check that

$$
\langle f, g\rangle_{4}=\left(f(x) e^{-x^{2} / 2}, g(x) e^{-x^{2} / 2}\right) .
$$

Orthonormal functions associated with the skew orthogonal polynomials are defined by

$$
\phi_{2 i}(x)=\frac{1}{\sqrt{h_{i}^{(4)}}} e^{-\frac{x^{2}}{2}} p_{2 i}(x), \quad \phi_{2 i+1}(x)=\frac{1}{\sqrt{h_{i}^{(4)}}} e^{-\frac{x^{2}}{2}} p_{2 i+1}(x)
$$

Relation (5.2.12) implies that

$$
\left(\phi_{2 i}, \phi_{2 j+1}\right) \equiv \frac{1}{2} \int d x\left(\phi_{2 i}(x) \phi_{2 j+1}^{\prime}(x)-\phi_{2 i+1}(x) \phi_{2 j}^{\prime}(x)\right)=\delta_{i j},
$$

and all other possible brackets are zeros. The following formula is well known, see Forrester, Nagao and Honner [29], Widom [58, and Adler, Forrester, Nagao, and van Moerbeke [1]

$$
\begin{aligned}
& \sum_{i=0}^{N-1}\left[\phi_{2 i}(x) \phi_{2 i+1}^{\prime}(y)-\phi_{2 i+1}(x) \phi_{2 i}^{\prime}(y)\right]= \\
& \frac{c_{2 N}}{c_{2 N-1}}\left[\frac{\psi_{2 N}(x) \psi_{2 N-1}(y)-\psi_{2 N-1}(x) \psi_{2 N}(y)}{x-y}+\psi_{2 N}(y) \int_{-\infty}^{x} \psi_{2 N-1}(t) d t\right],
\end{aligned}
$$

where the functions $\psi_{n}(x)$ are orthonormal functions associated with the Hermite polynomials (see equation (5.2.2)).

Proposition 5.2.3. The summation formula for the first kernel, $W_{N, I}^{(4)}(\zeta, \eta)$, is

$$
\begin{aligned}
W_{N, I}^{(4)}(\zeta, \eta) & =\frac{c_{2 N+2}}{c_{2 N+1}} e^{\frac{\zeta^{2}+\eta^{2}}{2}} \\
& \times\left[\int_{\eta}^{\zeta} d x \frac{\psi_{2 N+2}(\eta) \psi_{2 N+1}(x)-\psi_{2 N+1}(\eta) \psi_{2 N+2}(x)}{\eta-x}+\int_{\eta}^{\zeta} \psi_{2 N}(x) d x \int_{-\infty}^{\eta} \psi_{2 N-1}(t) d t\right]
\end{aligned}
$$

Proof. Application of basic summation formula (5.2.13) together with expression (5.1.5) for the kernel.

Proposition 5.2.4. It is possible to rewrite the functions $h_{2 i}(\eta), h_{2 i+1}(\eta)$ as follows

$$
\begin{aligned}
h_{2 i}(\eta) & =\sqrt{h_{i}^{(4)}} \int \frac{d x}{x-\eta} e^{-x^{2} / 2} \phi_{2 i}^{\prime}(x) \\
h_{2 i+1}(\eta) & =\sqrt{h_{i}^{(4)}} \int \frac{d x}{x-\eta} e^{-x^{2} / 2} \phi_{2 i+1}^{\prime}(x)
\end{aligned}
$$

Proof. Apply relation (5.2.12) and integrate by parts. 
Proposition 5.2.5. The second kernel, $W_{N, I I}^{(4)}(\zeta, \eta)$, can be rewritten as follows

$$
W_{N, I I}^{(4)}(\zeta, \eta)=e^{\zeta^{2} / 2} \frac{c_{2 N}}{c_{2 N-1}}\left[\frac{\Psi_{2 N}(\eta) \psi_{2 N-1}(\zeta)-\Psi_{2 N-1}(\eta) \psi_{2 N}(\zeta)}{\zeta-\eta}+\Psi_{2 N}(\eta) \int_{-\infty}^{\zeta} \psi_{2 N-1}(t) d t\right]
$$

where $\Psi_{N}$ was introduced by equation 5.2.4

Proof. We insert the expressions for the functions $h_{2 i}(\eta), h_{2 i+1}(\eta)$ obtained in Proposition 5.2.4 into the formula for the kernel $W_{N, I I}^{(4)}(\zeta, \eta)$, equation (5.1.6). After that we use

$$
\frac{1}{(y-\zeta)(y-\eta)}=\frac{1}{\zeta-\eta}\left(\frac{1}{y-\zeta}-\frac{1}{y-\eta}\right)
$$

and decompose the first resulting integral into two parts. Then we apply the well-known formula

$$
\Psi_{2 N}(\zeta) \psi_{2 N-1}(\zeta)-\Psi_{2 N-1}(\zeta) \psi_{2 N}(\zeta)=\frac{c_{2 N-1}}{c_{2 N}} e^{-\zeta^{2} / 2}
$$

and derive equation (5.2.17).

It is worth noting that the first term in the brackets of equation (5.2.17) is exactly the same as in the case of GOE, see (5.2.9).

Proposition 5.2.6. Let $\zeta \in \mathbb{C} \backslash \mathbb{R}, \eta \in \mathbb{C} \backslash \mathbb{R}$. The summation formula for the third kernel, $W_{N, I I I}^{(4)}(\zeta, \eta)$, is

$$
\begin{gathered}
W_{N, I I I}^{(4)}(\zeta, \eta)=\frac{c_{2 N-2}}{c_{2 N-3}}\left[\Psi_{2 N-3}(\eta) F_{2 N-2}(\eta, \zeta)-\Psi_{2 N-2}(\eta) F_{2 N-3}(\eta, \zeta)\right. \\
\left.-\Psi_{2 N-2}(\eta) \Psi_{2 N-3}(\zeta)\right]
\end{gathered}
$$

where we have introduced:

$$
F_{N}(\eta, \zeta)=\int \frac{d x \psi_{N}(x)}{\eta-x} \frac{d}{d x} \frac{e^{-x^{2} / 2}}{\zeta-x} .
$$

Proof. Equation (5.1.7) shows that we need to compute the following kernel:

$$
S_{N}^{I I I}(\zeta, \eta)=\sum_{i=0}^{N-2} \frac{h_{2 i+1}(\zeta) h_{2 i}(\eta)-h_{2 i}(\zeta) h_{2 i+1}(\eta)}{h_{i}^{(4)}}
$$

We rewrite the functions $h_{2 i+1}(\zeta), h_{2 i}(\zeta), h_{2 i+1}(\eta), h_{2 i}(\eta)$ explicitly using equation (5.2.15) and (5.2.16),

$$
\begin{aligned}
& h_{2 i}(\zeta)=\sqrt{h_{i}^{(4)}} \int d x \phi_{2 i}(x) \frac{d}{d x} \frac{e^{-x^{2} / 2}}{\zeta-x}, \quad h_{2 i+1}(\zeta)=\sqrt{h_{i}^{(4)}} \int d x \phi_{2 i+1}(x) \frac{d}{d x} \frac{e^{-x^{2} / 2}}{\zeta-x} \\
& h_{2 i}(\eta)=\sqrt{h_{i}^{(4)}} \int \frac{d x}{x-\eta} e^{-x^{2} / 2} \phi_{2 i}^{\prime}(x), \quad h_{2 i+1}(\eta)=\sqrt{h_{i}^{(4)}} \int \frac{d x}{x-\eta} e^{-x^{2} / 2} \phi_{2 i+1}^{\prime}(x) .
\end{aligned}
$$

Insert the above expressions to equation (5.2.21), and obtain

$$
S_{N}^{I I I}(\zeta, \eta)=\sum_{i=0}^{N-2} \iint d x d y\left[\frac{e^{-y^{2} / 2}}{\eta-y} \frac{d}{d x} \frac{e^{-x^{2} / 2}}{\zeta-x}\right]\left(\phi_{2 i+1}^{\prime}(y) \phi_{2 i}(x)-\phi_{2 i}^{\prime}(y) \phi_{2 i+1}(x)\right)
$$


Now we apply basic summation formula (5.2.13) and find

$$
\begin{array}{r}
S_{N}^{I I I}(\zeta, \eta)=\frac{c_{2 N-2}}{c_{2 N-3}}\left\{\iint d x d y\left[\frac{e^{-y^{2} / 2}}{\eta-y} \frac{d}{d x} \frac{e^{-x^{2} / 2}}{\zeta-x}\right]\left[\frac{\psi_{2 N-2}(x) \psi_{2 N-3}(y)-\psi_{2 N-3}(x) \psi_{2 N-2}(y)}{x-y}\right]\right. \\
\left.+\iint d x d y\left[\frac{e^{-y^{2} / 2}}{\eta-y} \frac{d}{d x} \frac{e^{-x^{2} / 2}}{\zeta-x}\right]\left[\psi_{2 N-2}(y) \int_{-\infty}^{x} \psi_{2 N-3}(t) d t\right]\right\} \\
\equiv \frac{c_{2 N-2}}{c_{2 N-3}}\left[I_{1}(\zeta, \eta)+I_{2}(\zeta, \eta)\right]
\end{array}
$$

Rewrite $I_{1}(\zeta, \eta)$ as a repeated integral

$$
I_{1}(\zeta, \eta)=\int d x \frac{d}{d x} \frac{e^{-x^{2} / 2}}{\zeta-x} \int d y \frac{e^{-y^{2} / 2}}{\eta-y} \frac{\psi_{2 N-2}(x) \psi_{2 N-3}(y)-\psi_{2 N-3}(x) \psi_{2 N-2}(y)}{x-y}
$$

Apply identity

$$
\frac{1}{(\eta-y)(x-y)}=\frac{1}{\eta-x}\left(\frac{1}{x-y}-\frac{1}{\eta-y}\right)
$$

in the inner integral of equation (5.2.22). Then we obtain, using equation (5.2.18),

$$
\begin{array}{r}
\int d y \frac{e^{-y^{2} / 2}}{\eta-y} \frac{\psi_{2 N-2}(x) \psi_{2 N-3}(y)-\psi_{2 N-3}(x) \psi_{2 N-2}(y)}{x-y} \\
=\frac{1}{\eta-x} \int d y e^{-y^{2} / 2} \frac{\psi_{2 N-2}(x) \psi_{2 N-3}(y)-\psi_{2 N-3}(x) \psi_{2 N-2}(y)}{x-y} \\
-\frac{1}{\eta-x} \int d y e^{-y^{2} / 2} \frac{\psi_{2 N-2}(x) \psi_{2 N-3}(y)-\psi_{2 N-3}(x) \psi_{2 N-2}(y)}{\eta-y} \\
=\frac{1}{\eta-x} e^{-x^{2} / 2}+\frac{1}{\eta-x}\left(\psi_{2 N-2}(x) \Psi_{2 N-3}(\eta)-\psi_{2 N-3}(x) \Psi_{2 N-2}(\eta)\right)
\end{array}
$$

Therefore,

or

$$
\begin{aligned}
I_{1}(\zeta, \eta) & =\int d x \frac{d}{d x} \frac{e^{-x^{2} / 2}}{\zeta-x} \frac{1}{\eta-x} e^{-x^{2} / 2}+ \\
& +\int d x \frac{d}{d x} \frac{e^{-x^{2} / 2}}{\zeta-x} \frac{1}{\eta-x}\left(\psi_{2 N-2}(x) \Psi_{2 N-3}(\eta)-\psi_{2 N-3}(x) \Psi_{2 N-2}(\eta)\right)
\end{aligned}
$$

$$
I_{1}(\zeta, \eta)=\left\langle\frac{1}{\eta-x}, \frac{1}{\zeta-x}\right\rangle+\Psi_{2 N-3}(\eta) F_{2 N-2}(\eta, \zeta)-\Psi_{2 N-2}(\eta) F_{2 N-3}(\eta, \zeta)
$$

We simplify $I_{2}(\zeta, \eta)$ by partial integration, obtain an expression for $S_{N}^{I I I}(\zeta, \eta)$, insert this to formula (5.1.7), and obtain (5.2.19).

\subsection{Asymptotics of functions associated with Hermite polynomials.}

5.3.1. Asymptotics of $\psi_{N}(x)$. The large $N$ asymptotics of the functions $\psi_{N}(x)$ in different regions of the real line can be found in Szegö [55], formula (8.22.14). However, the asymtotic expression near the edges of the spectrum is valid only in infinitesimally small neighborhoods (as $N \rightarrow \infty$ ) of the edges. In what follows we need uniform asymptotics of functions $\psi_{N}(x)$ on the entire real line. Theorem 2.2 in Deift, Kriecherbauer, McLaughlin and Zhou [23] provides the needed asymptotics of orthogonal polynomials with respect to the weights $w(x) d x=e^{-Q(x)} d x$ on the real line, where $Q(x)=\sum_{k=0}^{2 m} q_{k} x^{k}, q_{2 m}>0$ (see also Deift, Kriecherbauer, McLaughlin and Zhou [24]). The expressions in Theorem 2.2 are valid in the entire complex plane. We adopt this result to the particular case of $Q(x)=x^{2}$.

The parity of functions $\psi_{N}(x)$ defined by equation (5.2.2) corresponds to the parity of $N$, i. e. if $N$ is even, $\psi_{N}(x)$ is an even function, and if $N$ is odd, $\psi_{N}(x)$ is an odd function. Thus it is enough 
to consider the asymptotics of functions $\psi_{N}(x)$ on $\mathbb{R}_{+}$. We decompose $\mathbb{R}_{+}$into three regions: $B_{\delta}, C_{\delta}$

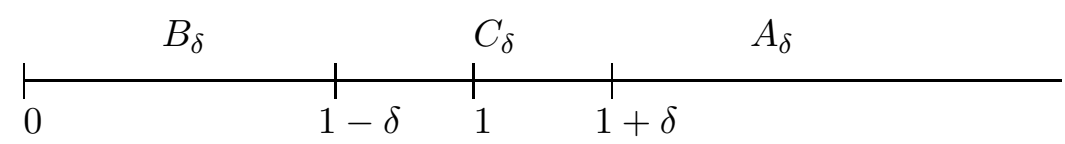

Fig. 4. Different asymptotic regions for $\psi_{N}(\sqrt{2 N} x)$

and $A_{\delta}$, see Fig. 4.

Theorem 5.3.1. (Plancherel-Rotach asymptotics of $\psi_{N}(\sqrt{2 N} x)$ on the real line.) There exists a $\delta_{0}$ such that for all $0<\delta \leq \delta_{0}$ the following asymptotic formulae hold:

(i) For $x \in A_{\delta}$

$$
\begin{aligned}
\psi_{N}(\sqrt{2 N} x) & =\frac{1}{2^{3 / 4} \pi^{1 / 2} N^{1 / 4}}\left[\left(\frac{x-1}{x+1}\right)^{1 / 4}+\left(\frac{x+1}{x-1}\right)^{1 / 4}\right] \\
& \times \exp \left[-\frac{\pi N}{2}\left[x \sqrt{x^{2}-1}-\ln \left(x+\sqrt{x^{2}-1}\right)\right]\right]\left(1+\mathcal{O}\left(\frac{1}{N}\right)\right) .
\end{aligned}
$$

(ii) For $x \in B_{\delta}$

$$
\begin{aligned}
\psi_{N}(\sqrt{2 N} x)= & \frac{2^{1 / 4}}{\pi^{1 / 2} N^{1 / 4}} \frac{1}{\left(1-x^{2}\right)^{1 / 4}}\left\{\cos \left(N x \sqrt{1-x^{2}}+(N+1 / 2) \arcsin x-\frac{N \pi}{2}\right)\right. \\
& \left.\times\left(1+\mathcal{O}\left(\frac{1}{N}\right)\right)+\sin \left(N x \sqrt{1-x^{2}}+(N-1 / 2) \arcsin x-\frac{N \pi}{2}\right) \mathcal{O}\left(\frac{1}{N}\right)\right\} .
\end{aligned}
$$

(iii) For $x \in C_{\delta}$

$$
\begin{aligned}
\psi_{N}(\sqrt{2 N} x)= & \frac{1}{(2 N)^{1 / 4}}\left\{\frac{(x+1)^{1 / 4}}{(x-1)^{1 / 4}}\left(f_{N}(x)\right)^{1 / 4} \operatorname{Ai}\left(f_{N}(x)\right)\left(1+\mathcal{O}\left(\frac{1}{N}\right)\right)-\right. \\
& \left.\frac{(x-1)^{1 / 4}}{(x+1)^{1 / 4}}\left(f_{N}(x)\right)^{-1 / 4} \operatorname{Ai}^{\prime}\left(f_{N}(x)\right)\left(1+\mathcal{O}\left(\frac{1}{N}\right)\right)\right\} .
\end{aligned}
$$

where the function $f_{N}(x)$ is given by

$$
f_{N}(x)=\left(\frac{3 N}{2}\right)^{2 / 3}\left[x \sqrt{x^{2}-1}-\ln \left(x+\sqrt{x^{2}-1}\right)\right]^{2 / 3},
$$

and $\mathrm{Ai}$ denotes the Airy function determined as the solution of

$$
\operatorname{Ai}^{\prime \prime}(x)=x \operatorname{Ai}(x)
$$

satisfying

$$
\lim _{x \rightarrow \infty} \operatorname{Ai}(x) \sqrt{4 \pi} x^{1 / 4} e^{\frac{2}{3} x^{3 / 2}}=1
$$


All the error terms are uniform for $\delta \in$ compact subsets of $\left(0, \delta_{0}\right]$ and for $x \in X_{\delta}$, where $X=$ $A, B, C$.

Remark 5.3.2. When $|x-1|<\epsilon, \epsilon$ is a small parameter, $f_{N}(x)=(x-1)\left(\phi_{N}(x)\right)^{2 / 3}$, where $\phi_{N}(x)$ is a strictly positive function at $x=1$. Therefore the function $\psi_{N}(\sqrt{2 N} x)$ is finite at $x=1$.

Corollary 5.3.3. $\sup _{x \in \mathbb{R}}\left|\psi_{N}(x)\right| \leq$ const $\cdot N^{-\frac{1}{12}}$.

Proof. This is evident from the asymptotic expressions above for $\psi_{N}(x)$, and from the asymptotic expansion of the Airy function and its derivative.

Proposition 5.3.4. Assume that $x \in[-M, M] ; M \rightarrow \infty$ as $N \rightarrow \infty, \frac{M}{\sqrt{N}} \rightarrow 0$ as $N \rightarrow \infty$. Then

$$
\psi_{N}(x)=\frac{2^{1 / 4}}{\pi^{1 / 2} N^{1 / 4}}\left[\cos \left(x \sqrt{2 N+1}-\frac{\pi N}{2}\right)+\mathcal{O}\left(\frac{M^{3}}{\sqrt{N}}\right)\right] .
$$

The error term is uniform for $x \in[-M, M]$.

Proof. This formula follows directly from equation (5.3.2), where we replace $x$ by $\frac{x}{\sqrt{2 N}}$ and expand in powers of $\frac{x}{\sqrt{2 N}}$ the fraction $\frac{1}{\left(1-\frac{x^{2}}{2 N}\right)^{1 / 4}}$, and the expression inside the cosine.

5.3.2. Asymptotics of $\Psi_{N}(\zeta)$. Recall that the Cauchy type transform $\Psi_{N}(\zeta)$ of the function $\psi_{N}(x)$ was defined by equation (5.2.4).

Proposition 5.3.5. Assume that $\zeta \in \mathbb{C} \backslash \mathbb{R}, M \rightarrow \infty$ as $N \rightarrow \infty, \ln N \ll M \ll \sqrt{N}$ as $N \rightarrow \infty$. Then the following asymptotic expression for $\Psi_{N}(\zeta)$ holds:

$$
\Psi_{N}(\zeta)=\frac{2^{1 / 4}}{\pi^{1 / 2} N^{1 / 4}} \int_{-M}^{M} \frac{d x e^{-x^{2} / 2}}{x-\zeta} \cos \left[x \sqrt{2 N+1}-\frac{\pi N}{2}\right]+\mathcal{O}\left(\frac{M^{3} \ln M}{N^{3 / 4}}\right) .
$$

The error term is uniform for all $\zeta$ taken from a compact subset of $\mathbb{C} \backslash \mathbb{R}$.

Proof. We decompose the integral in the definition of the function $\Psi_{N}(\zeta)$ (equation (5.2.4) ) into three parts:

$$
\int \frac{d x e^{-x^{2} / 2} \psi_{n}(x)}{x-\zeta}=\int_{-M}^{M} \frac{d x e^{-x^{2} / 2} \psi_{n}(x)}{x-\zeta}+\int_{M}^{+\infty} \frac{d x e^{-x^{2} / 2} \psi_{n}(x)}{x-\zeta}+\int_{-\infty}^{M} \frac{d x e^{-x^{2} / 2} \psi_{n}(x)}{x-\zeta} .
$$

By Corollary $5.3 .3 \psi_{N}(x)$ is uniformly bounded by const $\cdot N^{-1 / 12}$. This enables us to estimate the second and the third integrals in the expression above. Namely,

$$
\begin{aligned}
\left|\int_{M}^{+\infty} \frac{d x e^{-x^{2} / 2} \psi_{n}(x)}{x-\zeta}\right| & \leq \text { const } \cdot N^{-1 / 12} \int_{M}^{+\infty} d x e^{-x^{2} / 2} x \\
& =\text { const } \cdot N^{-1 / 12} e^{-M^{2} / 2}
\end{aligned}
$$

This shows that the second and third integrals in (5.3.9) are small. To obtain formula (5.3.8) we insert the asymptotic formula for $\psi_{N}(x)$ (equation (5.3.2) ) into the first integral in (5.3.9), and note that

$$
\int_{-M}^{M} \frac{d x e^{-x^{2} / 2}}{|x-\zeta|} \leq \int_{-M}^{M} \frac{d x}{|x-\zeta|}=\mathcal{O}(\ln M)
$$


Proposition 5.3.6. Under the same assumptions as in Proposition 5.3 .5 the asymptotic formula for the Cauchy type transform $\Psi_{N}(\zeta)$ of $\psi_{N}(x)$ is

$$
\Psi_{N}\left(\frac{\zeta}{\sqrt{2 N}}\right)=\frac{2^{1 / 4} \pi^{1 / 2}}{N^{1 / 4}}\left\{\begin{array}{ll}
i e^{i\left(\zeta-\frac{\pi N}{2}\right)}, & \operatorname{Im} \zeta>0, \\
-i e^{-i\left(\zeta-\frac{\pi N}{2}\right)}, & \operatorname{Im} \zeta<0
\end{array}+\mathcal{O}\left(\frac{M^{3} \ln M}{N^{3 / 4}}\right)\right.
$$

The error term is uniform for all $\zeta$ taken from a compact subset of $\mathbb{C} \backslash \mathbb{R}$.

Proof. We replace $x$ by $\frac{x}{\sqrt{2 N}}$ in the integral in equation (5.3.8). The obtained integral is finite as $N \rightarrow \infty$ and has the following limiting value

$$
\int_{-\infty}^{+\infty} \frac{d x}{x-\zeta} \cos \left[x-\frac{\pi N}{2}\right]
$$

We compute this integral by the standard residue calculations and get formula (5.3.11).

5.3.3. Asymptotics of $I_{N}\left(\frac{\eta}{\sqrt{2 N}}\right)$. The aim of this subsection is to prove the following Proposition:

Proposition 5.3.7. Let $\eta$ be taken from a compact subset $K$ of $\mathbb{C} \backslash \mathbb{R}$. Then the following estimate is valid

$$
\sup _{\eta \in K}\left|I_{N}\left(\frac{\eta}{\sqrt{2 N}}\right)\right| \leq \text { const } \cdot N^{-1 / 2}
$$

Proof. The function $I_{N}(\eta)$ can be rewritten as

$$
I_{N}(\eta)=\int_{-\infty}^{+\infty} d x \psi_{N}(x) \phi_{\eta}(x)
$$

where

$$
\phi_{\eta}(x)=\int_{-\infty}^{+\infty} d y e^{-y^{2} / 2} \operatorname{sgn}(y-x) \frac{1}{\eta-y} .
$$

Let $M \rightarrow \infty$ as $N \rightarrow \infty, \frac{M}{\sqrt{2 N}} \rightarrow 0$ as $N \rightarrow \infty$. Then the integral in the expression for $I_{N}(\eta)$ can be restricted to the domain $[-M, M]$, with an exponentially small error:

$$
I_{N}(\eta)=\int_{-M}^{M} d x \psi_{N}(x) \phi_{\eta}(x)+\mathcal{O}\left(e^{-M}\right) .
$$

In order to show this we note that the integral can be rewritten as follows

$$
I_{N}(\eta)=\int_{-\infty}^{+\infty} d y e^{-y^{2} / 2} \frac{1}{\eta-y} \int_{-\infty}^{+\infty} \operatorname{sgn}(y-x) \psi_{N}(x) d x
$$

We define

$$
\epsilon \psi_{N}(y)=\int_{-\infty}^{+\infty} \operatorname{sgn}(y-x) \psi_{N}(x) d x .
$$

Decompose the integral $I_{N}(\eta)$ into three parts:

$$
\begin{aligned}
I_{N}(\eta) & =\int_{-M}^{M} d y e^{-y^{2} / 2} \frac{1}{\eta-y} \epsilon \psi_{N}(y)+\int_{-\infty}^{-M} d y e^{-y^{2} / 2} \frac{1}{\eta-y} \epsilon \psi_{N}(y) \\
& +\int_{M}^{+\infty} d y e^{-y^{2} / 2} \frac{1}{\eta-y} \epsilon \psi_{N}(y) .
\end{aligned}
$$

The global estimate for $\epsilon \psi_{N}(y)$ is known (see, Nagao and Wadati [46], equation (A.1)):

$$
\sup _{y \in \mathbb{R}}\left|\epsilon \psi_{N}(y)\right| \leq \text { const } \cdot N^{-1 / 4}
$$


We use this estimate to evaluate the second and third integrals in equation (5.3.13). For example, for the third integral we obtain :

$$
\left|\int_{M}^{+\infty} d y e^{-y^{2} / 2} \frac{1}{\eta-y} \epsilon \psi_{N}(y)\right| \leq \frac{\text { const }}{N^{1 / 4}} \int_{M}^{+\infty} e^{-y^{2} / 2} y d y=\mathcal{O}\left(e^{-M}\right) .
$$

The same estimate is valid for the second integral in equation (5.3.13). By equation (5.3.12) the problem of evaluation of $I_{N}\left(\frac{\eta}{\sqrt{2 N}}\right)$ is reduced to the evaluation of the following integral:

$$
I_{N, M}(\eta)=\int_{-M}^{M} d x \psi_{N}(x) \phi_{\eta}(x)
$$

We change the variables of the integration, $x=t / \sqrt{2 N}, y=s / \sqrt{2 N}$, and find

$$
I_{N, M}\left(\frac{\eta}{\sqrt{2 N}}\right)=\frac{1}{\sqrt{2 N}} \int_{-M \sqrt{2 N}}^{M \sqrt{2 N}} d t \psi_{N}\left(\frac{t}{\sqrt{2 N}}\right) \int_{-\infty}^{+\infty} d s e^{-s^{2} / 4 N} \operatorname{sgn}(s-t) \frac{1}{\eta-s} .
$$

The last integral can be rewritten as

$$
I_{N, M}\left(\frac{\eta}{\sqrt{2 N}}\right)=\frac{1}{\sqrt{2 N}} \int_{-\infty}^{+\infty} d s e^{-s^{2} / 4 N} \frac{1}{\eta-s} \int_{-M \sqrt{2 N}}^{M \sqrt{2 N}} d t \psi_{N}\left(\frac{t}{\sqrt{2 N}}\right) \operatorname{sgn}(s-t) .
$$

Now we can insert the asymptotic expression for $\psi_{N}\left(\frac{t}{(2 N)^{1 / 2}}\right)$ into the second integral and obtain

$$
\int_{-M \sqrt{2 N}}^{M \sqrt{2 N}} d t \psi_{N}\left(\frac{t}{\sqrt{2 N}}\right) \operatorname{sgn}(s-t)=\frac{2^{1 / 4}}{\pi^{1 / 2} N^{1 / 4}} \int_{-M \sqrt{2 N}}^{M \sqrt{2 N}} d t \operatorname{sgn}(s-t)\left(\cos \left[t-\frac{\pi N}{2}\right]+\mathcal{O}\left(\frac{M^{3}}{\sqrt{2 N}}\right)\right) .
$$

This shows that the integral

$$
\int_{-M \sqrt{2 N}}^{M \sqrt{2 N}} d t \psi_{N}\left(\frac{t}{\sqrt{2 N}}\right) \operatorname{sgn}(s-t)
$$

is bounded by $\frac{\text { const } \cdot M^{4}}{N^{1 / 4}}$. The integral $\int_{-\infty}^{+\infty} d s e^{-s^{2} / 4 N} \frac{1}{|\eta-s|}$ is bounded by const $\cdot \log N$ uniformly for all $\eta \in K$, where $K$ is a compact subset of $\mathbb{C} \backslash \mathbb{R}$. Choosing $M=M(N)$ appropriately (such that $\frac{M^{4}(N) \log N}{N^{1 / 4}} \leq 1$ as $\left.N \rightarrow \infty\right)$ we prove the Proposition.

5.3.4. Estimate of $\int_{M}^{+\infty} \frac{\psi_{N}(x) d x}{x}$.

Proposition 5.3.8. Assume that $M \ll \sqrt{N}$. Then the following estimate is valid:

$$
\left|\int_{M}^{+\infty} \frac{\psi_{N}(x) d x}{x}\right| \leq \operatorname{Max}\left[\frac{\text { const }}{M^{2} N^{1 / 12}}, \frac{\text { const } M}{N^{3 / 4}}\right]
$$

where the constants do not depend on $N, M$.

Proof. We use representation of $\psi_{N}(x)$ in terms of the Hermite polynomial $H_{N}(x)$ (see equation (5.2.2) $)$, the relation $H_{N}^{\prime}(x)=2 N H_{N-1}(x)$, and take the integral $\int_{M}^{+\infty} \frac{\psi_{N}(x) d x}{x}$ by parts. As a 
result we obtain

$$
\int_{M}^{+\infty} \frac{\psi_{N}(x) d x}{x}=\frac{c_{N+1}}{(N+1) c_{N}}\left[-\frac{\psi_{N+1}(M)}{M^{2}}+\int_{M}^{+\infty} \psi_{N+1}(x) d x+\int_{M}^{+\infty} \frac{\psi_{N+1}(x) d x}{x^{2}}\right] .
$$

Since $\left|\psi_{N+1}(M)\right| \leq$ const $\cdot N^{-1 / 12}$ (Corollary [5.3.3) the first term in the brackets is estimated by const $\cdot N^{-1 / 12} M^{-2}$. As for the second term we note that

$$
\begin{aligned}
\left|\int_{M}^{+\infty} \psi_{N+1}(x) d x\right| & \leq\left|\int_{0}^{+\infty} \psi_{N+1}(x) d x\right|+\left|\int_{0}^{M} \psi_{N+1}(x) d x\right| \\
& \leq \operatorname{const} \cdot N^{-1 / 4}+\mathrm{const} \cdot N^{-1 / 4} M \leq \mathrm{const} \cdot N^{-1 / 4} M
\end{aligned}
$$

where we have used the estimate proved in Nagao an Wadati [46], equation (A.1):

$$
\left|\int_{0}^{+\infty} \psi_{N}(x) d x\right| \leq \mathrm{const} \cdot N^{-1 / 4}
$$

The third integral is evaluated as follows:

$$
\left|\int_{M}^{+\infty} \frac{\psi_{N+1}(x) d x}{x^{2}}\right| \leq \frac{1}{M^{2}} \int_{M}^{+\infty}\left|\psi_{N+1}(x)\right| d x \leq \frac{\operatorname{const} \sqrt{2 N}}{N^{1 / 12} M^{2}}
$$

Noting that the factor $\frac{c_{N+1}}{(N+1) c_{N}} \sim \frac{1}{\sqrt{2 N}}$, as $N \rightarrow \infty$, we obtain the estimate in the statement of the Proposition.

\subsubsection{Asymptotics of $E_{N}(\zeta, \eta)$.}

Proposition 5.3.9. Take $\zeta, \eta \in \mathbb{C} \backslash \mathbb{R}$. Then

$$
\begin{aligned}
E_{N}\left(\frac{\zeta}{\sqrt{2 N}}, \frac{\eta}{\sqrt{2 N}}\right)= & -\frac{2^{1 / 4}}{\pi^{1 / 2} N^{1 / 4}} \int_{\mathbb{R}} \frac{d y e^{-y^{2} / 4 N}}{\zeta-y} \int_{\mathbb{R}} \frac{d x \cos \left(x-\frac{\pi N}{2}\right) \operatorname{sgn}(y-x)}{\eta-x} \\
& +\mathcal{O}\left(\operatorname{Max}\left[\frac{M^{3} \log ^{2} N}{N^{3 / 4}}, \frac{\log N}{M^{2} N^{1 / 12}}\right]\right)
\end{aligned}
$$

as $N \rightarrow \infty$. The error term, $\mathcal{O}\left(\operatorname{Max}\left[\frac{M^{3} \log ^{2} N}{N^{3 / 4}}, \frac{\log N}{M^{2} N^{1 / 12}}\right]\right)$, is uniform for $\zeta, \eta$ taken from a compact subset of $\mathbb{C} \backslash \mathbb{R}$.

Remark 5.3.10. The error term $\mathcal{O}\left(\operatorname{Max}\left[\frac{M^{3} \log ^{2} N}{N^{3 / 4}}, \frac{\log N}{M^{2} N^{1 / 12}}\right]\right)$ in Proposition [5.3.9] is negligible in comparison with the main term,

$$
-\frac{2^{1 / 4}}{\pi^{1 / 2} N^{1 / 4}} \int_{\mathbb{R}} \frac{d y e^{-y^{2} / 4 N}}{\zeta-y} \int_{\mathbb{R}} \frac{d x \cos \left(x-\frac{\pi N}{2}\right) \operatorname{sgn}(y-x)}{\eta-x}
$$

if

$$
\frac{M^{3} \log ^{2} N}{N^{3 / 4}} \leq \frac{1}{N^{1 / 4}}, \quad \frac{\log N}{M^{2} N^{1 / 12}} \leq \frac{1}{N^{1 / 4}}
$$

Once $M=M(N)$ is chosen such that $N^{1 / 12}(\log N)^{1 / 2} \ll M \ll N^{1 / 6}(\log N)^{-2 / 3}$ the conditions above (equation (5.3.15) ) are satisfied. 
Proof. It is convenient to rewrite $E_{N}\left(\frac{\zeta}{\sqrt{2 N}}, \frac{\eta}{\sqrt{2 N}}\right)$ as

$$
E_{N}\left(\frac{\zeta}{\sqrt{2 N}}, \frac{\eta}{\sqrt{2 N}}\right)=\int \frac{e^{-y^{2} / 4 N} g_{N}\left(\frac{y}{\sqrt{2 N}}, \frac{\eta}{\sqrt{2 N}}\right) d y}{\zeta-y}
$$

where

$$
g_{N}\left(\frac{y}{\sqrt{2 N}}, \frac{\eta}{\sqrt{2 N}}\right)=\int \frac{\operatorname{sgn}(y-x) \psi_{N}\left(\frac{x}{\sqrt{2 N}}\right) d x}{x-\eta} .
$$

Equation (5.3.14) means that the function $\psi_{N-1}(x)$ in expression (5.3.16) can be replaced by its asymptotics in the center of the spectrum if we are interested in the scaling limit of $E_{N}(\zeta, \eta)$. In order to prove that such replacement is allowed we proceed as follows. First we will show that the integration over $y$ in equation (5.3.16) can be resticted to a domain on which the asymptotics of $\psi_{N-1}(x)$ is given by Proposition [5.3.4. Second we will observe that the integration over $x$ in equation (5.3.17) can be restricted to the same domain as well.

Let $K$ denote a compact subset of $\mathbb{C} \backslash \mathbb{R}$. The function $g_{N}\left(y, \frac{\eta}{\sqrt{2 N}}\right)$ has the following bound which is uniform in $y \in \mathbb{R}$ and in $\eta \in K$,

$$
\sup _{\eta \in \mathbb{K}}\left\{g_{N}\left(y, \frac{\eta}{\sqrt{2 N}}\right)\right\} \leq \text { const } \cdot N^{\alpha}, \quad \alpha-\text { real positive. }
$$

In order to prove this inequality we rewrite the integral in equation (5.3.17) as a sum of three integrals:

$$
\begin{aligned}
g_{N}\left(y, \frac{\eta}{\sqrt{2 N}}\right)= & \int_{-\sqrt{2 N}(1+\epsilon)}^{\sqrt{2 N}(1+\epsilon)} \frac{\operatorname{sgn}(y-x) \psi_{N}(x) d x}{x-\frac{\eta}{\sqrt{2 N}}}+\int_{\sqrt{2 N}(1+\epsilon)}^{+\infty} \frac{\operatorname{sgn}(y-x) \psi_{N}(x) d x}{x-\frac{\eta}{\sqrt{2 N}}} \\
& +\int_{-\infty}^{-\sqrt{2 N}(1+\epsilon)} \frac{\operatorname{sgn}(y-x) \psi_{N}(x) d x}{x-\frac{\eta}{\sqrt{2 N}}},
\end{aligned}
$$

where $\epsilon>0$. The following estimate is evident from the asymptotic expressions for the function $\psi_{N}(x)$ (see Section 5.3.1),

$$
\left|\psi_{N}(x)\right|<e^{-\operatorname{const} \sqrt{N}|x|}, \frac{|x|}{\sqrt{2 N}}>1+\epsilon, \epsilon>0
$$

Using this estimate we obtain that the second and the third integral in equation (5.3.19) are exponentially small. As for the first integral in equation (5.3.19) it is clearly polynomially bounded since $\left|x-\frac{\eta}{\sqrt{2 N}}\right| \geq \frac{\text { const }}{\sqrt{2 N}},(\operatorname{Im} \eta \neq 0)$, and $\sup _{x \in \mathbb{R}}\left|\psi_{N}(x)\right| \leq \frac{\text { const }}{N^{1 / 12}}$ (see Corollary [5.3.3). Thus inequality (5.3.18) is proved.

Let $M \rightarrow \infty$ as $N \rightarrow \infty$, but $\frac{M}{\sqrt{N}} \rightarrow 0$ as $N \rightarrow \infty$. Then we claim that

$$
E_{N}\left(\frac{\zeta}{\sqrt{2 N}}, \frac{\eta}{\sqrt{2 N}}\right)=\int_{-M}^{M} \frac{e^{-y^{2} / 2} g_{N}\left(y, \frac{\eta}{\sqrt{2 N}}\right) d y}{\frac{\zeta}{\sqrt{2 N}}-y}+\mathcal{O}\left(N^{\alpha} e^{-M^{2} / 2}\right)
$$


where the error term, $\mathcal{O}\left(N^{\alpha} e^{-M^{2} / 2}\right)$, is uniform for $\zeta, \eta$ taken from a compact subset of $\mathbb{C} \backslash \mathbb{R}$. Indeed, let us take $M$ as described above and rewrite $E_{N}\left(\frac{\zeta}{\sqrt{2 N}}, \frac{\eta}{\sqrt{2 N}}\right)$ as

$$
\begin{aligned}
E_{N}\left(\frac{\zeta}{\sqrt{2 N}}, \frac{\eta}{\sqrt{2 N}}\right)= & \int_{-M}^{M} \frac{e^{-y^{2} / 2} g_{N}\left(y, \frac{\eta}{\sqrt{2 N}}\right) d y}{\frac{\zeta}{\sqrt{2 N}}-y}+\int_{M}^{+\infty} \frac{e^{-y^{2} / 2} g_{N}\left(y, \frac{\eta}{\sqrt{2 N}}\right) d y}{\frac{\zeta}{\sqrt{2 N}}-y} \\
& +\int_{-\infty}^{-M} \frac{e^{-y^{2} / 2} g_{N}\left(y, \frac{\eta}{\sqrt{2 N}}\right) d y}{\frac{\zeta}{\sqrt{2 N}}-y}
\end{aligned}
$$

and estimate the second and the third integrals. Namely,

$$
\begin{aligned}
\left|\int_{M}^{+\infty} \frac{e^{-y^{2} / 2} g_{N}\left(y, \frac{\eta}{\sqrt{2 N}}\right) d y}{\frac{\zeta}{\sqrt{2 N}}-y}\right| & \leq\left|\int_{M}^{+\infty} e^{-y^{2} / 2} g_{N}\left(y, \frac{\eta}{\sqrt{2 N}}\right) y d y\right| \\
& \leq \operatorname{const} \cdot N^{\alpha} \int_{M}^{+\infty} e^{-y^{2} / 2} y d y=\text { const } \cdot N^{\alpha} \cdot e^{-M^{2} / 2} .
\end{aligned}
$$

where equation (5.3.18) was used. This shows that the integral over $y$ in the expression for $E_{N}\left(\frac{\zeta}{\sqrt{2 N}}, \frac{\eta}{\sqrt{2 N}}\right)$ can be restricted to the domain $[-M, M]$ with a small error term.

Now assume that $y$ is taken from the interval $[-M, M]$, and $M=M(N)$ is chosen such that $M \rightarrow \infty$ as $N \rightarrow \infty$, but $\frac{M}{\sqrt{N}} \rightarrow 0$ as $N \rightarrow \infty$. Then

$$
\int_{-\infty}^{+\infty} \frac{\operatorname{sgn}(y-x) \psi_{N}(x) d x}{x-\frac{\eta}{\sqrt{2 N}}}=\int_{-M}^{M} \frac{\operatorname{sgn}(y-x) \psi_{N}(x) d x}{x-\frac{\eta}{\sqrt{2 N}}}+\mathcal{O}\left\{\operatorname{Max}\left[M N^{-3 / 4}, M^{-2} N^{-1 / 12}\right]\right\}
$$

as $N \rightarrow \infty$. The error term, $\mathcal{O}\left\{\operatorname{Max}\left[M N^{-3 / 4}, M^{-2} N^{-1 / 12}\right]\right\}$, is uniform on $y \in \mathbb{R}, \eta \in \mathbb{C} \backslash \mathbb{R}$, where $\eta$ is taken from a compact subset of $\mathbb{C} \backslash \mathbb{R}$. In order to prove (5.3.20) we decompose the integral in the left-hand side of equation (5.3.20) into three integrals on intervals $[-M, M],(-\infty,-M)$, and $(M,+\infty)$. The integrals on intervals $(-\infty,-M)$ and $(M,+\infty)$ are evaluated using Proposition 5.3 .8 .

To summarize we have proved that

$$
\begin{aligned}
E_{N}\left(\frac{\zeta}{\sqrt{2 N}}, \frac{\eta}{\sqrt{2 N}}\right)= & \int_{-M}^{M} \frac{e^{-y^{2} / 2} d y}{\frac{\zeta}{\sqrt{2 N}}-y}\left\{\int_{-M}^{M} \frac{\operatorname{sgn}(y-x) \psi_{N}(x) d x}{x-\frac{\eta}{\sqrt{2 N}}}+\mathcal{O}\left[\operatorname{Max}\left[M N^{-3 / 4}, M^{-2} N^{-1 / 12}\right]\right]\right\} \\
& +\mathcal{O}\left(N^{\alpha} e^{-M^{2} / 2}\right) .
\end{aligned}
$$


The error term $\mathcal{O}\left(N^{\alpha} e^{-M^{2} / 2}\right)$ is exponentially small. Integrating the error term $\mathcal{O}\left[\operatorname{Max}\left[\frac{M}{N^{3 / 4}}, \frac{1}{M^{2} N^{1 / 12}}\right]\right]$ we obtain:

$$
\begin{aligned}
& \mid \int_{-M}^{M} \frac{e^{-y^{2} / 2} d y}{\frac{\zeta}{\sqrt{2 N}}-y} \mathcal{O}\left[\operatorname{Max}\left[M N^{-3 / 4}, M^{-2} N^{-1 / 12}\right]\right]= \\
& =\left|\int_{-M \sqrt{2 N}}^{M \sqrt{2 N}} \frac{e^{-y^{2} / 4 N} d y}{\zeta-y} \mathcal{O}\left[\operatorname{Max}\left[M N^{-3 / 4}, M^{-2} N^{-1 / 12}\right]\right]\right| \leq \\
& \leq \mathcal{O}\left[\operatorname{Max}\left[M \log N N^{-3 / 4}, M^{-2} \log N N^{-1 / 12}\right]\right] .
\end{aligned}
$$

Another error term is due to the replacement of $\psi_{N}(x)$ by its asymptotics taken from Proposition 5.3.4. This error term is evaluated as follows:

$$
\left|\int_{-M \sqrt{2 N}}^{M \sqrt{2 N}} \frac{e^{-y^{2} / 4 N} d y}{\zeta-y} \int_{-M \sqrt{2 N}}^{M \sqrt{2 N}} \frac{d x}{x-\eta} \mathcal{O}\left[\frac{M^{3}}{N^{3 / 4}}\right]\right| \leq \mathcal{O}\left[\frac{M^{3}(\log N)^{2}}{N^{3 / 4}}\right] .
$$

This completes the proof.

\subsubsection{Asymptotics of $F_{N}(\zeta, \eta)$.}

Proposition 5.3.11. Let $\zeta, \eta$ be chosen from a compact subset of $\mathbb{C} \backslash \mathbb{R}$. Then

$$
\begin{aligned}
F_{N}\left(\frac{\eta}{2 \sqrt{N}}, \frac{\zeta}{2 \sqrt{N}}\right)=2 N\left[\frac{2^{1 / 4}}{\pi^{1 / 2} N^{1 / 4}}\right. & \int_{-M}^{M} d t \cos \left(t-\frac{\pi N}{2}\right)\left[\frac{1}{\eta-t} \frac{d}{d t} \frac{e^{-t^{2} / 4 N}}{\zeta-t}\right] \\
& \left.+\mathcal{O}\left(\operatorname{Max}\left[\frac{M^{4}}{N^{3 / 4}}, \frac{1}{N^{1 / 12} M^{2}}\right]\right)\right]
\end{aligned}
$$

where $M=M(N)$ is chosen such that $M(N) \rightarrow \infty$ as $N \rightarrow \infty$, but $\frac{M(N)}{\sqrt{N}} \rightarrow 0$ as $N \rightarrow \infty$.

Proof. Recall that $F_{N}(\zeta, \eta)$ was defined by an integral in equation (5.2.20). Change the variable of integration in this integral, and decompose it into three integrals. The first one runs from $-\infty$ to $-M$, the second one runs from $-M$ to $M$, and the third one runs from $M$ to $+\infty$. In the second integral replace the function $\psi_{N}(x)$ by its asymptotics in the bulk of the spectrum (see Proposition 5.3.4). The integration of the error term gives $\mathcal{O}\left(\frac{M^{4}}{N^{3 / 4}}\right)$. The first and the third integrals are of $\operatorname{order} \mathcal{O}\left(\frac{1}{M^{2} N^{1 / 12}}\right)$. It can be seen rewriting the expression $\frac{1}{\eta-t} \frac{d}{d t} \frac{e^{-t^{2} / 4 N}}{\zeta-t}$ explicitly, and using the fact that $\sup _{t \in R}\left|\psi_{N}(t)\right| \leq \mathrm{const} \cdot N^{-1 / 12}$ (Corollary [5.3.3).

Remark 5.3.12. The error term, $\mathcal{O}\left(\operatorname{Max}\left[\frac{M^{4}}{N^{3 / 4}}, \frac{1}{N^{1 / 12} M^{2}}\right]\right)$, is negligible in comparison with the first term in the brackets of equation (5.3.21), if the following conditions are satisfied:

$$
\frac{M^{4}}{N^{3 / 4}} \ll \frac{1}{N^{1 / 4}}, \frac{1}{N^{1 / 12} M^{2}} \ll \frac{1}{N^{1 / 4}} .
$$

The first condition implies that $M \ll N^{1 / 8}$, and the second equation implies that $N^{1 / 12} \ll M$. Therefore if $M(N)$ is chosen such that $N^{1 / 12} \ll M \ll N^{1 / 8}$ the error term is negligible in comparison with the main term.

\subsection{Asymptotics of the kernels: GOE case.}


5.4.1. Asymptotics of $W_{N, I}^{(1)}\left(\frac{\zeta}{\sqrt{2 N}}, \frac{\eta}{\sqrt{2 N}}\right)$.

Theorem 5.4.1. (Scaling limit of the first kernel) For $\zeta \in \mathbb{C}, \eta \in \mathbb{C}$,

$$
\lim _{N \rightarrow \infty}\left\{\frac{1}{2 N} W_{N, I}^{(1)}\left(\frac{\zeta}{\sqrt{2 N}}, \frac{\eta}{\sqrt{2 N}}\right)\right\}=-\frac{1}{\pi} \frac{d}{d \zeta}\left[\frac{\sin (\zeta-\eta)}{\zeta-\eta}\right] .
$$

Proof. Use formula (5.2.5), the following relation which follows immediately from the properties of the Hermite polynomials,

$$
\frac{d}{d x} \psi_{n}(x)=n \frac{c_{n-1}}{c_{n}} \psi_{n-1}(x)-x \psi_{n}(x)
$$

together with the asymptotic formula for the functions $\psi_{n}(x)$, Proposition 5.3.6.

5.4.2. Asymptotics of $W_{N, I I}^{(1)}\left(\frac{\zeta}{\sqrt{2 N}}, \frac{\eta}{\sqrt{2 N}}\right)$.

Proposition 5.4.2. Take $\zeta, \eta \in \mathbb{C} \backslash \mathbb{R}$. Then

$$
\lim _{N \rightarrow \infty}\left\{\sqrt{2 N} \frac{\Psi_{N}\left(\frac{\eta}{\sqrt{2 N}}\right) \psi_{N-1}\left(\frac{\zeta}{\sqrt{2 N}}\right)-\Psi_{N-1}\left(\frac{\eta}{\sqrt{2 N}}\right) \psi_{N}\left(\frac{\zeta}{\sqrt{2 N}}\right)}{\zeta-\eta}\right\}= \begin{cases}\frac{2}{\zeta-\eta} e^{i(\eta-\zeta)}, & \operatorname{Im} \eta>0, \\ \frac{2}{\zeta-\eta} e^{-i(\eta-\zeta)}, & \operatorname{Im} \eta<0\end{cases}
$$

Proof. Apply Propositions 5.3.4 and 5.3.6.

Theorem 5.4.3. (Scaling limit of the second kernel) Let $\zeta \in \mathbb{C}, \eta \in \mathbb{C} \backslash \mathbb{R}$. Then

$$
\lim _{N \rightarrow \infty} \frac{1}{\sqrt{2 N}} W_{N, I I}^{(1)}\left(\frac{\zeta}{\sqrt{2 N}}, \frac{\eta}{\sqrt{2 N}}\right)= \begin{cases}\frac{e^{i(\eta-\zeta)}}{\zeta-\eta} & \operatorname{Im} \eta>0 \\ \frac{e^{-i(\eta-\zeta)}}{\zeta-\eta} & \operatorname{Im} \eta<0\end{cases}
$$

Proof. This follows immediately from equation (5.2.9), Propositions 5.4.2, 5.3.7, and from the fact that $\frac{c_{N}}{c_{N-1}} \sim \sqrt{\frac{N}{2}}$ as $N \rightarrow \infty$.

5.4.3. Asymptotics of $W_{N, I I I}^{(1)}\left(\frac{\zeta}{\sqrt{2 N}}, \frac{\eta}{\sqrt{2 N}}\right)$.

Theorem 5.4.4. (Scaling limit of the third kernel) Take $\zeta \in \mathbb{C} \backslash \mathbb{R}, \eta \in \mathbb{C} \backslash \mathbb{R}$. Then

$\lim _{N \rightarrow \infty} W_{N, I I I}^{(1)}\left(\frac{\zeta}{\sqrt{2 N}}, \frac{\eta}{\sqrt{2 N}}\right)=2 \pi i \begin{cases}\int_{1}^{+\infty} \frac{e^{i(\zeta-\eta) t} d t}{t} & \operatorname{Im} \zeta>0, \operatorname{Im} \eta<0 \\ -\int_{1}^{+\infty} \frac{e^{-i(\zeta-\eta) t} d t}{t} & \operatorname{Im} \zeta<0, \operatorname{Im} \eta>0, \quad \zeta \in \mathbb{C} \backslash \mathbb{R}, \quad \eta \in \mathbb{C} \backslash \mathbb{R} . \\ 0 & \text { otherwise. }\end{cases}$

Proof. Recall that the kernel $W_{N, I I I}^{(1)}(\zeta, \eta)$ is given by equation (5.2.10). In order to determine the large $N$ asymptotics of $W_{N, I I I}^{(1)}(\zeta, \eta)$ consider the expression:

$$
A_{N}(\zeta, \eta) \equiv E_{N-1}(\zeta, \eta) \Psi_{N-2}(\zeta)-E_{N-2}(\zeta, \eta) \Psi_{N-1}(\zeta)
$$

The asymptotics for $E_{N}(\zeta, \eta)$ (equation (5.3.14)), and the asymptotics for $\Psi_{N}(\zeta)$ (equation (5.3.11)) give us the leading term of $A_{N}(\zeta, \eta)$ :

$$
A_{N}(\zeta, \eta) \simeq \begin{cases}\sqrt{\frac{2}{N}} \int_{\mathbb{R}} \frac{d y e^{-y^{2} / 4 N}}{\eta-y} \int_{\mathbb{R}} \frac{e^{i(\zeta-x)} \operatorname{sgn}(y-x)}{\zeta-x}, & \operatorname{Im} \zeta>0 \\ -\sqrt{\frac{2}{N}} \int_{\mathbb{R}} \frac{d y e^{-y^{2} / 4 N}}{\eta-y} \int_{\mathbb{R}} \frac{e^{-i(\zeta-x)} \operatorname{sgn}(y-x)}{\zeta-x}, & \operatorname{Im} \zeta<0\end{cases}
$$


In what follows the integral representation for $\operatorname{sgn}(y-x)$ will play a role,

$$
\operatorname{sgn}(y-x)=\frac{1}{i \pi} \int_{\mathbb{R}} \frac{e^{i(y-x) t} d t}{t} .
$$

If we insert this expression to equation (5.4.2), and perform the computations formally (i. e. changing the order of integrations, and replacing $e^{-y^{2} / 4 N}$ by 1$)$ we obtain:

$$
A_{N}\left(\frac{\zeta}{\sqrt{2 N}}, \frac{\eta}{\sqrt{2 N}}\right) \simeq 2 \pi i\left\{\begin{array}{cl}
\frac{4}{\sqrt{2 N}} \int_{1}^{+\infty} \frac{e^{i(\zeta-\eta) t} d t}{t} & \operatorname{Im} \zeta>0, \operatorname{Im} \eta<0 \\
-\frac{4}{\sqrt{2 N}} \int_{1}^{+\infty} \frac{e^{-i(\zeta-\eta) t} d t}{t} & \operatorname{Im} \zeta<0, \operatorname{Im} \eta>0 \\
0 & \text { otherwise. }
\end{array}\right.
$$

Since the factor $\frac{1}{2} \frac{c_{N-1}}{c_{N-2}} \simeq \frac{\sqrt{N}}{2 \sqrt{2}}$, as $N \rightarrow \infty$, and the term $I_{N-1}\left(\frac{\zeta}{\sqrt{2 N}}\right) I_{N-2}\left(\frac{\zeta}{\sqrt{2 N}}\right)$ is negligible in the large $N$ limit in comparison with $A_{N}\left(\frac{\zeta}{\sqrt{2 N}}, \frac{\eta}{\sqrt{2 N}}\right)$ (see Proposition [5.3.7), formula (55.4.1) follows.

However these computations involve the integrals which are not absolutely convergent, so formula (5.4.3) must be justified. In order to prove this formula rigorously we observe that the following holds:

$$
\int_{\mathbb{R}} \frac{e^{i(\zeta-x)} \operatorname{sgn}(y-x)}{\zeta-x}=2 \int_{1}^{+\infty} \frac{e^{-i(y-\zeta) t} d t}{t}, \quad \operatorname{Im} \zeta>0
$$

Indeed, the integral in the left-hand side of equation (5.4.4) is equal to $2 \int_{y}^{+\infty} \frac{d x e^{i(\zeta-x)}}{x-\zeta}$ (since $\int_{-\infty}^{+\infty} \frac{d x e^{i(\zeta-x)}}{x-\zeta}=0$, when $\left.\operatorname{Im} \zeta>0\right)$. To check that $\int_{y}^{+\infty} \frac{d x e^{i(\zeta-x)}}{x-\zeta}$ is equal to $\int_{1}^{+\infty} \frac{e^{-i(y-\zeta) t} d t}{t}$ we first observe that the derivatives with respect to $y$ are equal to each other. Second, it is evident that the first integral tends to zero as $y \rightarrow+\infty$. The fact that the second integral tends to zero when $y \rightarrow+\infty$ can be proved by partial integration. Thus it remains to compute the large $N$ limit of the expression

$$
\int_{\mathbb{R}} \frac{d y e^{-y^{2} / 4 N}}{\eta-y} \int_{1}^{+\infty} \frac{e^{-i(y-\zeta) t} d t}{t}, \operatorname{Im} \zeta>0 .
$$

The integrand, as a function in $(y, t)$, decays exponentially as $\max (|y|,|t|) \rightarrow \infty$ Therefore we are allowed to change the order of integration, and expression (5.4.5) is equal to

$$
\int_{1}^{+\infty} \frac{e^{i \zeta t} d t}{t} \int_{\mathbb{R}} \frac{d y e^{-i y t-y^{2} / 4 N}}{\eta-y}, \operatorname{Im} \zeta>0 .
$$

The large $N$ limit of the inner integral can be computed as follows. Take $M=M(N)$ such that $M(N) \rightarrow \infty$ as $N \rightarrow \infty$, but $M^{2}(N) / N \rightarrow 0$ as $N \rightarrow \infty$. Decompose the inner integral into three integrals: the first one runs from $-\infty$ to $-M$, the second one runs from $-M$ to $M$, and the third one runs from $M$ to $+\infty$. Then the first and the third integrals are of order $\mathcal{O}\left(\frac{1}{M}\right)$. As for the second integral, it asymptotically equals to $(2 \pi i) e^{-i \eta t}$, when $\operatorname{Im} \eta<0$, and to zero, when $\operatorname{Im} \eta>0$. It can be observed considering the semicircle contour $C_{M} \cup[-M, M]$, where $C_{M}$ is a semicircle from the point $M$ to the point $-M$ passing through lower half of the complex plane. The Jordan Lemma implies that

$$
\left|\int_{C_{M}} d z \frac{e^{-z^{2} / 4 N} e^{-i z t}}{\eta-z}\right| \leq \frac{\text { const } e^{-M^{2} / 4 N}}{M \cdot t}
$$


Since $t \geq 1$ we obtain that

$$
\int_{C_{M}} d z \frac{e^{-z^{2} / 4 N} e^{-i z t}}{\eta-z}=\mathcal{O}\left(\frac{1}{M}\right)
$$

where the estimate is uniform for $t \in[1,+\infty)$. Thus the inner integral in equation (5.4.6) is determined by the residue at $\eta$ of the integrand (uniformly for $t \in[1,+\infty$ ) with error of order $\mathcal{O}\left(\frac{1}{M}\right)$ ). Replacing the inner integral by this residue we again arrive to formula (5.4.3).

\subsection{Asymptotics of the kernels: GSE case.}

\subsubsection{Asymptotic of the first kernel.}

Theorem 5.5.1. (Scaling limit of the first kernel) For complex $\zeta, \eta$

$$
\lim _{N \rightarrow \infty} W_{N, I}^{(4)}\left(\frac{\zeta}{2 \sqrt{N}}, \frac{\eta}{2 \sqrt{N}}\right)=\frac{1}{\pi} \int_{0}^{1} d t \frac{\sin (\zeta-\eta) t}{t}
$$

Proof. Propositions 5.2.3 gives exact formula for the kernel $W_{N, I}^{(4)}$ (equation (5.2.14)). The second term in the brackets in equation (5.2.14) is the product of two integrals. The first integral is of order $\mathcal{O}\left(N^{-3 / 4}\right)$, since $\left|\psi_{2 N}\left(\frac{u}{2 \sqrt{N}}\right)\right| \leq$ const $\cdot N^{-1 / 4}$ uniformly for $u$ chosen from a compact real interval. (Note that the asymptotics of functions $\psi_{N}$ in a real neighborhood of zero can be extended to a complex neighborhood of zero, see Szegö [55]; Deift, Kriecherbauer, McLaughlin, Venakides and Zhou [23], such that the bound $\left|\psi_{2 N}\left(\frac{u}{2 \sqrt{N}}\right)\right| \leq$ const $\cdot N^{-1 / 4}$ remains valid). The second integral is bounded by const $\cdot N^{-1 / 4}$ (see Nagao and Wadati [46], equation (A.1)). Thus the second term in the brackets in equation (5.2.14) (where $\zeta$ is replaced by $\frac{\zeta}{2 \sqrt{N}}$, and $\eta$ is replaced by $\frac{\eta}{2 \sqrt{N}}$ ) is of order $\mathcal{O}\left(N^{-1}\right)$. As for the first term in the brackets of equation (5.2.14) it is of order $\mathcal{O}\left(N^{-1 / 2}\right)$. Indeed, this term is an integral over a compact interval, so the functions $\psi_{2 N+2}, \psi_{2 N+1}$ can be replaced by their asymptotic expressions ( Proposition 5.3.4) in the bulk of the spectrum. Thus we observe that the second term in the brackets in equation (5.2.14) is negligible with respect to the first term, and formula (5.5.1) is obtained.

\subsubsection{Asymptotics of the second kernel.}

Theorem 5.5.2. (Scaling limit of the second kernel) Let $\zeta \in \mathbb{C}$. Then

$$
\lim _{N \rightarrow \infty} \frac{1}{2 \sqrt{N}} W_{N, I I}^{(4)}\left(\frac{\zeta}{2 \sqrt{N}}, \frac{\eta}{2 \sqrt{N}}\right)= \begin{cases}\frac{e^{i(\eta-\zeta)}}{\zeta-\eta} & \operatorname{Im} \eta>0 \\ \frac{e^{-i(\eta-\zeta)}}{\zeta-\eta} & \operatorname{Im} \eta<0\end{cases}
$$

Proof. The exact expression for the second kernel in the case of GSE is essentially the same as in the case of GOE. Thus the asymptotics is obtained by the same method.

\subsubsection{Asymptotics of the third kernel.}

Theorem 5.5.3. (Scaling limit of the third kernel) Let $\zeta, \eta$ are chosen from a compact subset of $\mathbb{C} \backslash \mathbb{R}$. Then

$$
\lim _{N \rightarrow \infty}\left[\frac{1}{4 N} W_{N, I I I}^{(4)}\left(\frac{\zeta}{2 \sqrt{N}}, \frac{\eta}{2 \sqrt{N}}\right)\right]=2 \pi i\left\{\begin{array}{cl}
e^{i \eta} \frac{d}{d \zeta}\left(\frac{e^{-i \zeta}}{\eta-\zeta}\right) & \operatorname{Im} \zeta<0, \operatorname{Im} \eta>0 \\
-e^{-i \eta} \frac{d}{d \zeta}\left(\frac{e^{i \zeta}}{\eta-\zeta}\right) & \operatorname{Im} \zeta>0, \operatorname{Im} \eta<0 \\
0 & \text { otherwise. }
\end{array}\right.
$$


Proof. Consider equation (5.2.19). Replace the functions $\Psi_{2 N-2}, \Psi_{2 N-3}, F_{2 N-2}, F_{2 N-3}$ by their asymptotics given by equations (5.3.11) and (5.3.21). The first two terms in the brackets of equation (5.2.19) are then represented by an integral which can be directly computed by residue calculations. As for the third term in the brackets of equation (5.2.19) it is asymptotically negligible in comparison with two first terms.

Remark 5.5.4. The asymptotics of the kernels $W_{N, I}^{(2)}(\alpha, \beta), W_{N, I I}^{(2)}(\alpha, \beta), W_{N, I I I}^{(2)}(\alpha, \beta)$ is determined in Ref. [53. Note that this asymptotics can be obtained exploiting the asymptotic expressions for $\psi_{N}$ ( Proposition 5.3.4) and for $\Psi_{N}$ (Proposition 5.3.6).

\section{Appendix. Some useful facts from the Linear Algebra}

6.1. The formula for the Cauchy determinant. Let $A=\left(a_{1}, \ldots, a_{k}\right), B=\left(b_{1}, \ldots, b_{k}\right)$ be two non-intersecting sets. Then the following formula is valid:

$$
\operatorname{det}\left(\frac{1}{a_{i}-b_{j}}\right)_{i, j=1}^{k}=(-)^{\frac{k(k-1)}{2}} \frac{V(A) V(B)}{\prod(A ; B)}
$$

6.2. Determinant of the block matrix. If the matrix $H$ has the following block structure,

$$
H=\left(\begin{array}{cc}
0 & A \\
B & 0
\end{array}\right)
$$

with square matrices $A$ and $B$, then

$$
\operatorname{det} H=(-)^{|A| \cdot|B|} \operatorname{det} A \cdot \operatorname{det} B .
$$

6.3. Minors of the inverse matrix. Assume that two $N \times N$ matrices $A$ and $B$ are inverse to each other,

$$
A=B^{-1}
$$

Then an arbitrary minor of the matrix $A$ corresponding to the rows $\alpha_{1}, \ldots, \alpha_{r}$ and columns $\beta_{1}, \ldots, \beta_{r}$ can be expressed as follows:

$$
\begin{gathered}
\operatorname{det} A\left(\alpha_{1}, \ldots, \alpha_{r} \mid \beta_{1}, \ldots, \beta_{r}\right)= \\
=\left(-\sum_{i=1}^{\sum_{i=1}^{r} \alpha_{i}+\beta_{i}} \frac{\operatorname{det} B\left(1, \ldots, \check{\beta_{1}}, \ldots, \check{\beta}_{r}, \ldots, N \mid 1, \ldots, \check{\alpha_{1}}, \ldots, \check{\alpha_{r}}, \ldots, N\right)}{\operatorname{det} B}\right.
\end{gathered}
$$

Here $\check{k}$ denotes the $k^{\text {th }}$ row (or column) which is removed from the matrix.

6.4. The expansion of the minors of the matrix $I+A$. Let $\check{\alpha}=\left(\check{\alpha_{1}}, \ldots, \check{\alpha_{r}}\right), \check{\beta}=\left(\check{\beta_{1}}, \ldots, \check{\beta_{r}}\right)$ denote removed columns or rows. Assume that $\check{\alpha} \cap \breve{\beta}$ is empty. Then the following expansion of the minor of the matrix $I+A$ can be obtained:

$$
\begin{gathered}
\operatorname{det}(I+A)\left(1, \ldots, \check{\alpha_{1}}, \ldots, \check{\alpha_{r}}, \ldots, N \mid 1, \ldots, \check{\beta_{1}}, \ldots, \check{\beta_{r}}, \ldots, N\right)= \\
(-)^{\sum_{i=1}^{r} \alpha_{i}+\beta_{i}+r} \sum_{X} \operatorname{det} A\left(\beta_{1}, \ldots, \beta_{r}, X \mid \alpha_{1}, \ldots, \alpha_{r}, X\right)
\end{gathered}
$$

Here the sum is over all subsets $X$ of the set $[1, \ldots, N]$, which do not intersect $\alpha$ and $\beta$. 
6.5. The minors of the matrix $K$. Applying two previous expressions it is easy to relate the minors of the matrix $K$ defined in terms of the $L$ matrix of the $L$-ensemble as

$$
K=I-\frac{I}{I+L}
$$

with minors of the matrix $L$. Namely we have

$$
\operatorname{det} K(\alpha \mid \beta)=\sum_{X} \frac{\operatorname{det} L(\alpha, X \mid \beta, X)}{\operatorname{det}(I+L)}
$$

where $\alpha=\left(\alpha_{1}, \ldots, \alpha_{r}\right), \beta=\left(\beta_{1}, \ldots, \beta_{r}\right), \alpha_{i} \neq \beta_{j}$. Here again the sum is over all subsets $X$ of the set $[1, \ldots, N]$.

6.6. Definition of Pfaffian. The Pfaffian of a $2 N \times 2 N$ antisymmetric matrix $A=\left\|A_{j k}\right\|_{j, k=1}^{2 N}$ is defined as

$$
\operatorname{Pf} A=\sum_{\substack{\sigma=\left(i_{1}, \ldots, i_{2 N}\right) \in S_{2 N} \\ i_{1}<i_{2}, \ldots, i_{2 N-1}<i_{2 N} \\ i_{1}<i_{3}<\ldots<i_{2 N-1}}} \operatorname{sgn}(\sigma) A_{i_{1} i_{2}} \ldots A_{i_{2 N-1} i_{2 N}}
$$

6.7. Pfaffian of the block matrix. The following formula is valid

$$
\operatorname{Pf}\left[\begin{array}{cc}
0 & A \\
-A^{\dagger} & 0
\end{array}\right]=\operatorname{det} A(-)^{\frac{|A|(|A|-1)}{2}}
$$

6.8. The expansion of Pfaffians of submatrices of the matrix $J+A$. Let A be a $2 N \times 2 N$ antisymmetric matrix,

$$
\begin{aligned}
& A\left[1^{\prime}, 1^{\prime \prime}, \ldots, N^{\prime}, N^{\prime \prime} \mid 1^{\prime}, 1^{\prime \prime}, \ldots, N^{\prime}, N^{\prime \prime}\right]= \\
& \left.\qquad \begin{array}{ccccc}
0 & A\left(1^{\prime}, 1^{\prime \prime}\right) & \ldots & A\left(1^{\prime}, N^{\prime}\right) & A\left(1^{\prime}, N^{\prime \prime}\right) \\
-A\left(1^{\prime}, 1^{\prime \prime}\right) & 0 & & A\left(1^{\prime \prime}, N^{\prime}\right) & A\left(1^{\prime \prime}, N^{\prime \prime}\right) \\
\vdots & & & & \\
-A\left(1^{\prime}, N^{\prime}\right) & -A\left(1^{\prime}, N^{\prime \prime}\right) & 0 & A\left(N^{\prime}, N^{\prime \prime}\right) \\
-A\left(1^{\prime}, N^{\prime \prime}\right) & -A\left(1^{\prime \prime}, N^{\prime \prime}\right) & -A\left(N^{\prime}, N^{\prime \prime}\right) & 0
\end{array}\right]
\end{aligned}
$$

and $J$ be the antisymmetric matrix of format $2 N \times 2 N$ defined by

$$
J=\left[\begin{array}{ccccccc}
0 & 1 & 0 & 0 & \ldots & 0 & 0 \\
-1 & 0 & 0 & 0 & & 0 & 0 \\
0 & 0 & 0 & 1 & & 0 & 0 \\
0 & 0 & -1 & 0 & & 0 & 0 \\
\vdots & & & & & & \\
0 & 0 & 0 & 0 & \ldots & 0 & 1 \\
0 & 0 & 0 & 0 & \ldots & -1 & 0
\end{array}\right]
$$

then the following expansion is valid $(2 \leq 2 k \leq N)$ :

$$
\begin{aligned}
& \operatorname{Pf}(J+A)\left[1^{\prime}, 2^{\prime}, \ldots, 2 k^{\prime} \mid 1^{\prime}, 2^{\prime}, \ldots, 2 k^{\prime}\right]= \\
& \sum_{\substack{X=\left(x_{1}^{\prime}, x_{1}^{\prime \prime}, \ldots, x_{d}^{\prime}, x_{d}^{\prime \prime}\right) \\
\left(x_{1}, \ldots x_{d}\right) \subset(2 k+1, \ldots, N)}} \operatorname{Pf} A\left[1^{\prime \prime}, 2^{\prime \prime}, \ldots, 2 k^{\prime \prime}, X \mid 1^{\prime \prime}, 2^{\prime \prime}, \ldots, 2 k^{\prime \prime}, X\right]
\end{aligned}
$$


6.9. The Pfaffian of a submatrix of the inverse matrix. With $A$ defined by equation (6.8.1) consider the inverse matrix $B=A^{-1}$. For $2 \leq 2 m \leq N$ we find

$$
\begin{aligned}
& \operatorname{Pf} B\left[1^{\prime}, 2^{\prime}, \ldots, 2 m^{\prime} \mid 1^{\prime}, 2^{\prime}, \ldots, 2 m^{\prime}\right]=\frac{1}{\operatorname{Pf} A} \\
& \times \operatorname{Pf} A\left[1^{\prime \prime}, \ldots, 2 m^{\prime \prime}, 2 m+1^{\prime}, 2 m+1^{\prime \prime}, \ldots, N^{\prime}, N^{\prime \prime} \mid\right. \\
& \left.1^{\prime \prime}, \ldots, 2 m^{\prime \prime}, 2 m+1^{\prime}, 2 m+1^{\prime \prime}, \ldots, N^{\prime}, N^{\prime \prime}\right]
\end{aligned}
$$

\section{REFERENCES}

[1] Adler, M.; Forrester, P.J.; Nagao, T.; van Moerbeke, P. Classical skew orthogonal polynomials and random matrices. J. Stat. Phys. 99 (1-2), (2000) 141-170.

[2] Akemann, G.; Fyodorov, Y. V. Universal random matrix correlations of ratios of characteristic polynomials at the spectral edges. Nucl. Phys. B 664, (2003) 457-476.

[3] Akemann, G.; Vernizzi, G. Characteristic polynomials of complex random matrix models. Nucl. Phys. B 660 (2003) 532-556.

[4] Andreev, A. V.; Simons, B. D. Correlators of spectral determinants in Quantum Chaos. Phys. Rev. Lett. 75 (12), (1995) 2304-2307.

[5] Baik, J.; Deift, P.; Strahov, E. Products and ratios of characteristic polynomials of random hermitian matrices. J. Math. Phys. 44, (2003) 3657-3670.

[6] Baik, J.; Kriecherbauer, T.; McLaughlin, K. T-R.; Miller, P. D. Uniform asymptotics for polynomials orthogonal with respect to a general class of discrete weights and universality results for associated ensembles: Announcement of results. International Mathematical Research Notices 15, (2003) 821-858. math.CA/0310278

[7] Baik, J.; Rains, E. Algebraic aspects of increasing subsequences. Duke Math. J.,109, (2001) 1-65.

[8] Bleher, P.; Its, A. Semiclassical asymptotics of orthogonal polynomials, Riemann-Hilbert problem, and universality in the matrix model. Ann. Math. 150(2), (1999) 185-266.

[9] Borodin, A. Duality of orthogonal polynomials on a finite set. J. Stat. Phys. 109(5-6), (2002) 1109-1120.

[10] Borodin, A.; Okounkov, A.; Olshanski, G. Asymptotics of Plansherel measures for symmetric groups. J. Amer. Math. Soc. 13 (2000), 491-515.

[11] Borodin, A.; Olshanski, G. Distributions on partitions, point processes and the hypergeometric kernel. Comm. Math. Phys. 211 (2000), no.2, 335-358.

[12] Borodin, A.; Olshanski, G. Harmonic analysis on the infinite-dimensional unitary group and determinantal point processes, math/0109194 (to appear in Ann. Math.)

[13] Borodin, A.; Olshanski, G. Z-measures on partitions and their scaling limits. math-ph/0210048 (to appear in Europ. Jour. Comb. )

[14] Borodin, A.; Olshanski, G. Random partitions and the Gamma kernel. math-ph/0305043 (to appear in Adv. Math.)

[15] Brezin, E.; Hikami, S. Characteristic polynomials of random matrices. Comm. Math. Phys. 214 (1), (2000) 111-135.

[16] Brezin, E.; Hikami, S. Charcteristic polynomials of random matrices at edge singularities. Phys. Rev. E 62(3), (2000) 3558-3567.

[17] Brezin E.; Hikami, S. Characteristic polynomials of real symmetric random matrices. Comm. Math. Phys. 223 (2), (2001) 363-382.

[18] Brezin E.; Hikami, S. New correlation functions for random matrices and integrals over supergroups. J. Phys. A. 36 (3), (2003) 711-751.

[19] de Bruijn N., G.; On some multiple integrals involving determinants. J. Indian. Math. Soc. (N.S.), 19 (1955) 1333-151.

[20] Conrey, J., B.; Farmer, D.,W.; Keating, J.,P.; Rubinshtein, M., O.; Snaith, N. C. Autocorrelation of random matrix polynomials. Comm. Math. Phys. 237 (3), (2003) 365-395.

[21] Daley, D., J.; Vere-Jones, D. An introduction to the theory of point processes. Springer series in statistics, Springer, 1988.

[22] Deift, P. Orthogonal Polynomials and Random Matrices: A Riemann-Hilbert Approach. Courant lecture notes, 3 New York: Courant Institute of Mathematical sciences, New York University 2000.

[23] Deift, P.; Kriecherbauer, T.; McLaughlin, K. T-R.; Venakides, S.; Zhou, X., A. Strong asymptotics of orthogonal polynomials with respect to exponential weights. Comm. Pure Appl. Math. 52 (12), (1999) 1491-1552.

[24] Deift, P.; Kriecherbauer, T.; McLaughlin, K. T-R.; Venakides, S.; Zhou, X., A. Uniform asymptotics for polynomials orthogonal with respect to varying exponential weights and applications to universality questions in random matrix theory. Comm. Pure Appl. Math. 52 (11), (1999) 1335-1425.

[25] Deift, P.; Gioev, D. (in preparation) 
[26] Eynard, B. Asymptotics of skew orthogonal polynomials. J. Phys. A. 34, (2001) 7591-7605.

[27] Forrester, P., J. Log Gases and Random Matrices. (http //:www.ms.unimelb.edu.au/ matpjfmatpjf.html).

[28] Forrester, P., J.; Keating, J., P. Singularity dominated strong fluctuations for some random matrix averages. math-ph/0402001

[29] Forrester, P., J.; Nagao, T.; Honner, G. Correlations for the orthogonal-unitary and symplectic-unitary transitions at the hard and soft edges. Nucl. Phys. B 553 (3), 601-643 1999.

[30] Fyodorov, Y., V. Negative moments of characteristic polynomials of random matrices: Ingham-Siegel integral as an alternative to Hubbard-Stratonovich transformation. Nucl. Phys. B 621 (3), (2002) 643-674.

[31] Fyodorov, Y., V. Complexity of Random Energy Landscapes, Glass Transition and Absolute Value of Spectral Determinant of Random Matrices. Phys. Rev. Lett. 92 (2004), 240601.

[32] Fyodorov, Y., V.; Akemann, G. On the supersymmetric partition function in QCD-inspired random matrix models. JETP. Lett. 77 (8), (2003) 438-441.

[33] Fyodorov, Y., V.; Keating, J., P. Negative moments of characteristic polynomials of random GOE matrices and singularity-dominated strong fluctuations. J. Phys. A. 36 (2003) 4035-4046.

[34] Fyodorov, Y., V.; Strahov, E. An exact formula for general correlation function of random Hermitian matrices. J. Phys. A. 36, (2003) $3203-3213$.

[35] Fyodorov, Y., V.; Strahov, E. On correlation functions of characteristic polynomials for chiral Gaussian unitary ensemble Nucl. Phys. B 647 (3), (2002) 581-597.

[36] Fyodorov, Y., V.; Strahov, E. Characteristic polynomials of random Hermitian matrices and DuistermaatHeckman localisation on non-compact Kahler manifolds. Nucl. Phys. B 630 (3), (2002) 453-491.

[37] Gronqvist, J.; Guhr, T.; Kohler, H. The k-point random matrix kernels obtained from one-point supermatrix models. J. Phys. A. 37 (6), (2004) 2331-2344.

[38] Hughes, C., P.; Keating, J., P., O'Connell, N. On the characteristic polynomial of a random unitary matrix. Comm. Math. Phys. 220 (2), (2001) 429-451.

[39] Johansson, K. Non-intersecting paths, random tilings and random matrices. Probability Theory and Related Fields. 123(2), (2002) 225-280.

[40] Keating, J., P.; Snaith, N., C. Random Matrix Theory and zeta(1/2+it). Comm. Math. Phys. 214 (1), (2000) $57-89$.

[41] Keating, J., P.; Snaith, N., C. Random matrices and L-functions. J. Phys. A. 36 (12), (2003) $2859-2881$.

[42] Keating, J., P.; Snaith, N., C. Random matrix theory and L-functions at s=1/2. Comm. Math. Phys. 214 (1), (2000) 91-110.

[43] Kerov, S.; Okounkov, A.; Olshanski, G. The boundary of the Young graph with Jack edge multiplicities. Int. Math. Res. Notes. 4, (1998) 173-199.

[44] Mehta, M. Random Matrices, 2nd ed. (Academic, San Diego, 1991)

[45] Mehta, M., L.; Normand, J., M. Moments of the characteristic polynomial in the three ensembles of random matrices. J. Phys. A. 34(22), (2001) 4627-4639.

[46] Nagao, T.; Forrester, P., J. Vicious random walkers and a discretization of Gaussian random matrix ensembles. Nucl. Phys. B. 620 (3) (2002) 551-565.

[47] Nagao, T.,; Wadati, M: Correlations Functions of random Matrix Ensembles Related to Classical Orthogonal Polynomials. III J. Phys. Soc. Japan, 61 (1992) 1910-1918.

[48] Olshanski, G. Pfaffian processes, unpublished note (2002)

[49] Rains, E. Correlation functions for symmetrized increasing subsequences, math. CO/0006097.

[50] Soshnikov, A. Determinantal random point fields. Russian Math. Surveys 55, (2000) 923-975

[51] Soshnikov, A. Janossy densities. II. Pfaffian ensembles. J. Stat. Phys. 113 (3-4), (2003) 611-622.

[52] Splittorff, K.; Verbaarschot, J., J., M. Replica limit of the Toda lattice equation. Phys. Rev. Lett. 90(4), (2003) art. no. 041601.

[53] Strahov, E.; Fyodorov, Y., V.Universal Results for Correlations of Characteristic Polynomials: Riemann-Hilbert Approach. Comm. Math. Phys. 241, (2003) 343-382.

[54] Szabo, R., J. Microscopic spectrum of the QCD Dirac operator in three dimensions. Nucl. Phys. B 598 (1-2), (2001) 309-347.

[55] Szegö, G. Orthogonal Polynomials, Vol.23 of American Mathematical Society, Colloquium Publications, 4th ed. (AMS, Providence, RI, 1975).

[56] Tracy, C., A.; Widom, H. Correlation functions, claster functions, and spacing distributions of random matrices. J. Stat. Phys. 92, (1998) 809-835.

[57] Vanlessen, M.,. Universal behaviour for averages of characteristic polynomials at the origin of the spectrum. math-ph/0306078

[58] Widom, H. On the relation between orthogonal, symplectic and unitary random matrices. J. Stat. Phys. 94, (1999) 347-364.

[59] Zirnbauer, M., R. Dual pairs in Random Matrix Theory. Talk given at LMS Workshop: Zeta Functions, Random Matrices and Quantum Chaos. September 13-14, 2001.

[60] Zirnbauer, M., R. Random Matrices, Symmetry Classes, and Dual Pairs. Talk given at James H. Simons Workshop on Random Matrix Theory. Stony Brook, February 20-23, 2002. 


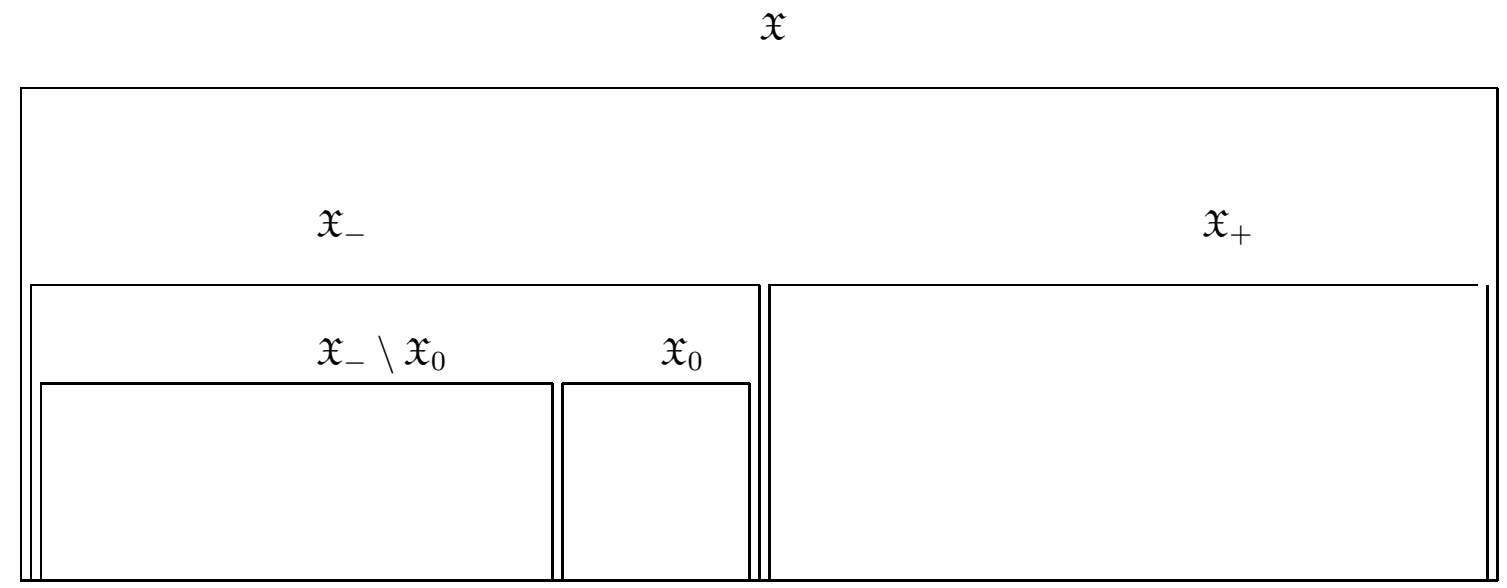

Fig 1. The decomposition of the discrete set $\mathfrak{X}$ 


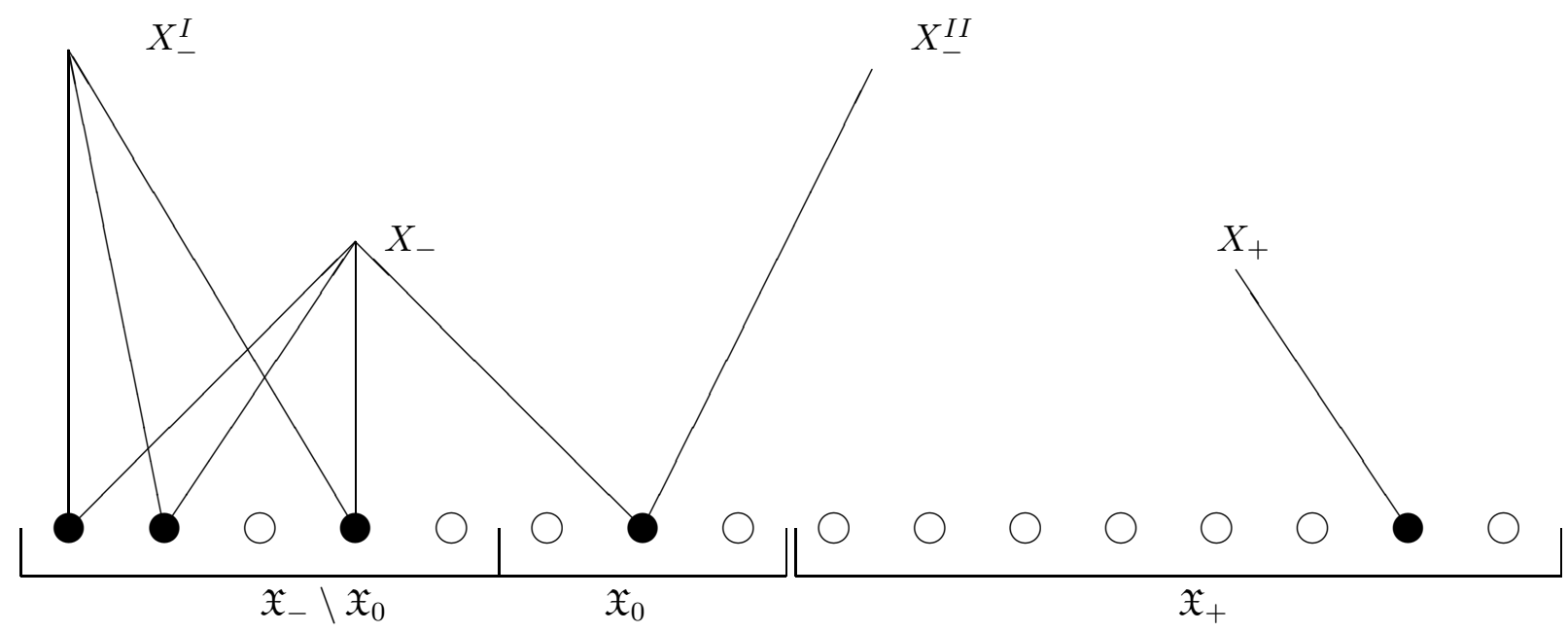

Fig 2. Unbalanced particle-particle configurations. The configuration $X$ consists of four negative particles and one positive particle. The set $\mathfrak{X}_{0}$ is chosen such that $\left|\mathfrak{X}_{0}\right|=\left|X_{-}\right|-\left|X_{+}\right|$. 


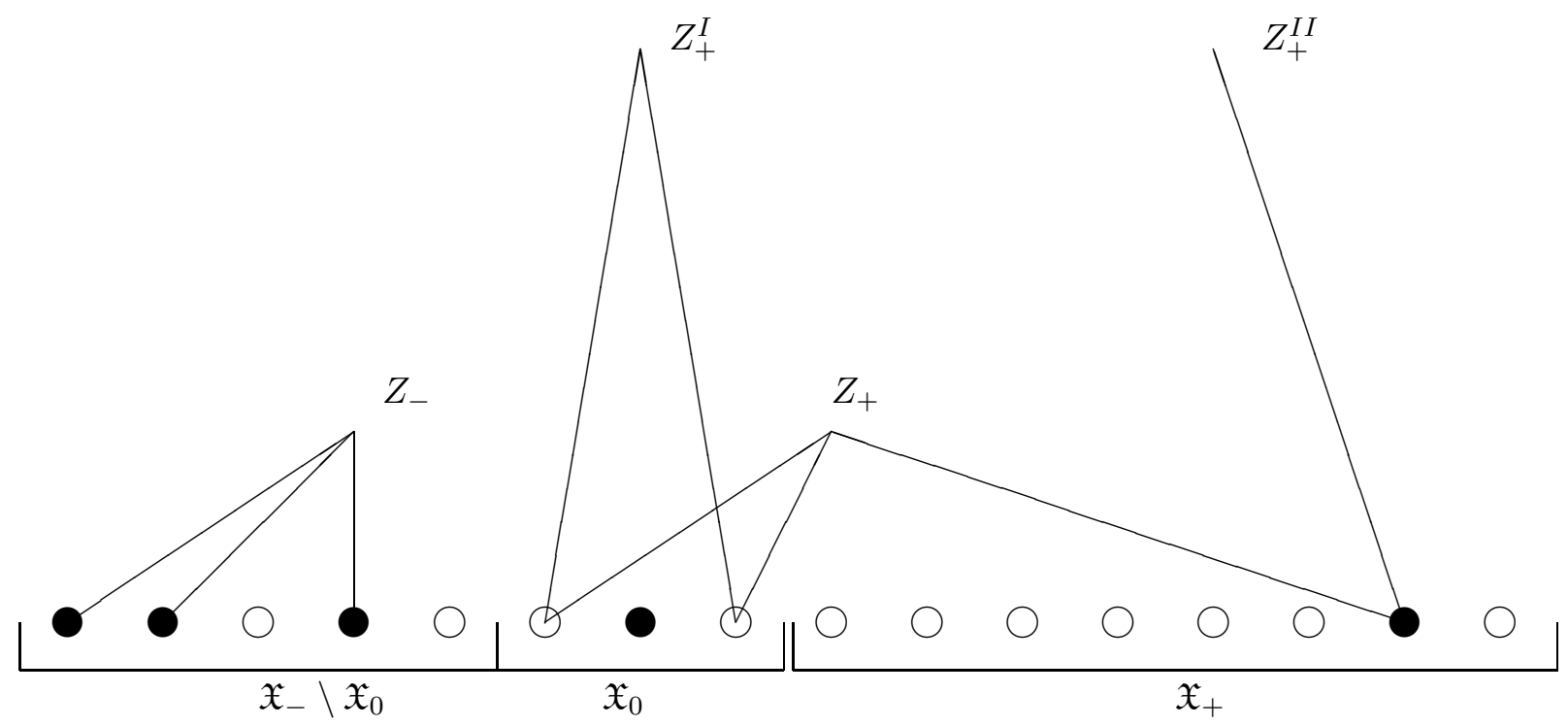

Fig 3. Balanced particle-hole configurations. $Z_{-}$consists of three negative particles, $\left|Z_{-}\right|=3$. $Z_{+}$ consists of one positive particle, $\left|Z_{+}^{I I}\right|=1$, and two holes in $\mathfrak{X}_{0},\left|Z_{+}^{I}\right|=2$ 


\begin{tabular}{|c|c|c|}
\hline Splitting of $\mathfrak{X}$ & $\begin{array}{c}\mathfrak{X}=\left(\mathfrak{X}_{-} \mid \mathfrak{X}_{+}\right)=\left(\mathfrak{X}_{-} \backslash \mathfrak{X}_{0} \sqcup \mathfrak{X}_{0} \mid \mathfrak{X}_{+}\right) \\
\left|\mathfrak{X}_{0}\right|=S\end{array}$ & $\mathfrak{X}=\left(\hat{\mathfrak{X}}_{-} \mid \hat{\mathfrak{X}}_{+}\right)=\left(\mathfrak{X}_{-} \backslash \mathfrak{X}_{0} \mid \mathfrak{X}_{0} \sqcup \mathfrak{X}_{+}\right)$ \\
\hline Point Configurations & $\begin{array}{c}X=\left(X_{-} \mid X_{+}\right)=\left(X_{-}^{I} \sqcup X_{-}^{I I} \mid X_{+}\right) \\
X_{-}^{I}=X_{-} \cap\left(\mathfrak{X}_{-} \backslash \mathfrak{X}_{0}\right), \quad X_{-}^{I I}=X_{-} \cap \mathfrak{X}_{0}\end{array}$ & $\begin{array}{l}Z=\left(Z_{-} \mid Z_{+}\right)=\left(Z_{-} \mid Z_{+}^{I} \sqcup Z_{+}^{I I}\right) \\
Z_{+}^{I}=Z_{+} \cap \mathfrak{X}_{0}, Z_{+}^{I I}=Z_{+} \cap \mathfrak{X}_{+}\end{array}$ \\
\hline$L$-ensembles & $\begin{array}{c}L=\left(\begin{array}{cc}0 & A \\
-A^{T} & 0\end{array}\right) \\
A(x, y)=\frac{h(x) h(y)}{x-y}\end{array}$ & $\begin{array}{c}\hat{L}=\left(\begin{array}{cc}0 & \hat{A} \\
-\hat{A}^{T} & 0\end{array}\right) \\
\hat{A}(x, y)=\frac{\hat{h}(x) \hat{h}(y)}{x-y}\end{array}$ \\
\hline $\begin{array}{c}\text { Relations } \\
\text { between configurations }\end{array}$ & $\begin{array}{c}X_{-}^{I}=Z_{-} \\
X_{-}^{I I}=\mathfrak{X}_{0} \backslash Z_{+}^{I} \\
\quad X_{+}=Z_{+}^{I I}\end{array}$ & $\begin{array}{l}Z_{-}=X_{-}^{I} \\
Z_{+}^{I}=\mathfrak{X}_{0} \backslash X_{-}^{I I} \\
\quad Z_{+}^{I I}=X_{+}\end{array}$ \\
\hline
\end{tabular}

TABLE $1 . L$-ensemble and $\hat{L}$-ensemble 


\begin{tabular}{|c|c|c|c|c|}
\hline & $L$-ensemble & $\triangle_{N}$-ensemble & $\hat{L}$-ensemble & $\hat{\triangle}_{N+S}$-ensemble \\
\hline $\begin{array}{l}\text { Splitting of } \\
\qquad \mathfrak{X}\end{array}$ & $\begin{array}{c}\mathfrak{X}=\left(\mathfrak{X}_{-} \mid \mathfrak{X}_{+}\right) \\
=\left(\mathfrak{X}_{-} \backslash \mathfrak{X}_{0} \sqcup \mathfrak{X}_{0} \mid \mathfrak{X}_{+}\right) \\
\left|\mathfrak{X}_{0}\right|=S ;\left|\mathfrak{X}_{+}\right|=N\end{array}$ & $\mathfrak{X}=\left(\mathfrak{X}_{-} \mid \mathfrak{X}_{+}\right)$ & $\begin{array}{c}\mathfrak{X}=\left(\hat{\mathfrak{X}}_{-} \mid \hat{\mathfrak{X}}_{+}\right) \\
=\left(\mathfrak{X}_{-} \backslash \mathfrak{X}_{0} \mid \mathfrak{X}_{0} \sqcup \mathfrak{X}_{+}\right) \\
\left|\hat{\mathfrak{X}}_{+}\right|=N+S\end{array}$ & $\mathfrak{X}=\left(\hat{\mathfrak{X}}_{-} \mid \hat{\mathfrak{X}}_{+}\right)$ \\
\hline $\begin{array}{c}\text { Point } \\
\text { configurations }\end{array}$ & $\begin{array}{l}X=\left(X_{-} \mid X_{+}\right) \\
X_{-}=X \cap \mathfrak{X}_{-} \\
X_{+}=X \cap \mathfrak{X}_{+}\end{array}$ & $X^{\triangle}=\left(X_{-} \mid \mathfrak{X}_{+} \backslash X_{+}\right)$ & $\begin{array}{l}Z=\left(Z_{-} \mid Z_{+}\right) \\
Z_{-}=Z \cap \hat{\mathfrak{X}}_{-} \\
Z_{+}=Z \cap \hat{\mathfrak{X}}_{+}\end{array}$ & $Z^{\triangle}=\left(Z_{-} \mid \hat{\mathfrak{X}}_{+} \backslash Z_{+}\right)$ \\
\hline Number of points & arbitrary $\leq N$ & $N$ & arbitrary $\leq N+S$ & $N+S$ \\
\hline Weight & $h$ & $f$ & $\hat{h}$ & $\hat{f}=f$ \\
\hline
\end{tabular}

TABLE 2. $L$-ensemble and polynomial ensemble

Department of Mathematics, 253-37, Caltech, Pasadena, CA 91125

E-mail address: borodin@caltech.edu, strahov@caltech.edu 